\title{
Gravity-mode period spacings as seismic diagnostic for a sample of $\gamma$ Doradus stars from Kepler space photometry and high-resolution ground-based spectroscopy ${ }^{1}$
}

\author{
T. Van Reeth, A. Tkachenko² \\ Instituut voor Sterrenkunde, KU Leuven, Celestijnenlaan 200D, 3001 Leuven, Belgium \\ timothy.vanreeth@ster.kuleuven. be \\ C. Aerts \\ Instituut voor Sterrenkunde, KU Leuven, Celestijnenlaan 200D, 3001 Leuven, Belgium \\ Department of Astrophysics, IMAPP, University of Nijmegen, PO Box 9010, 6500 GL Nijmegen, The \\ Netherlands \\ Kavli Institute for Theoretical Physics, University of California, Santa Barbara, CA 93106, USA \\ P. I. Pápics ${ }^{3}$ \\ Instituut voor Sterrenkunde, KU Leuven, Celestijnenlaan 200D, 3001 Leuven, Belgium \\ Kavli Institute for Theoretical Physics, University of California, Santa Barbara, CA 93106, USA \\ S. A. Triana \\ Instituut voor Sterrenkunde, KU Leuven, Celestijnenlaan 200D, 3001 Leuven, Belgium \\ K. Zwintz \\ Instituut voor Sterrenkunde, KU Leuven, Celestijnenlaan 200D, 3001 Leuven, Belgium \\ Institute for Astro- and Particle Physics, University of Innsbruck, Technikerstrasse 25/8, A-6020 Innsbruck, \\ Austria \\ P. Degroote, J. Debosscher \\ Instituut voor Sterrenkunde, KU Leuven, Celestijnenlaan 200D, 3001 Leuven, Belgium \\ S. Bloemen
}

\footnotetext{
${ }^{1}$ Based on data gathered with the NASA Discovery mission Kepler and spectra obtained with the HERMES spectrograph, which is installed at the Mercator Telescope, operated on the island of La Palma by the Flemish Community at the Spanish Observatorio del Roque de los Muchachos of the Instituto de Astrofísica de Canarias, and supported by the Fund for Scientific Research of Flanders (FWO), Belgium, the Research Council of KU Leuven, Belgium, the Fonds National de la Recherche Scientifique (F.R.S.-FNRS), Belgium, the Royal Observatory of Belgium, the Observatoire de Genève, Switzerland, and the Thüringer Landessternwarte Tautenburg, Germany. All data used in this study are downloadable from https://fys.kuleuven.be/ster/Projects/gamma-doradus
} 
Department of Astrophysics, IMAPP, University of Nijmegen, PO Box 9010, 6500 GL Nijmegen, The Netherlands

Kavli Institute for Theoretical Physics, University of California, Santa Barbara, CA 93106, USA

V. S. Schmid ${ }^{4}$

Instituut voor Sterrenkunde, KU Leuven, Celestijnenlaan 200D, 3001 Leuven, Belgium

Kavli Institute for Theoretical Physics, University of California, Santa Barbara, CA 93106, USA

K. De Smedt

Instituut voor Sterrenkunde, KU Leuven, Celestijnenlaan 200D, 3001 Leuven, Belgium

Y. Fremat

Royal Observatory of Belgium, Ringlaan 3, 1180 Brussels, Belgium

A. S. Fuentes, W. Homan

Instituut voor Sterrenkunde, KU Leuven, Celestijnenlaan 200D, 3001 Leuven, Belgium

M. Hrudkova, R. Karjalainen

Isaac Newton Group of Telescopes, Apartado de Correos 321, E-38700 Santa Cruz de la Palma, Canary Islands, Spain

R. Lombaert

Instituut voor Sterrenkunde, KU Leuven, Celestijnenlaan 200D, 3001 Leuven, Belgium

P. Nemeth

Dr. Karl Remeis-Observatory E ECAP, Astronomisches Inst., FAU Erlangen-Nuremberg, 96049 Bamberg, Germany

R. Østensen

Instituut voor Sterrenkunde, KU Leuven, Celestijnenlaan 200D, 3001 Leuven, Belgium

G. Van De Steene

Royal Observatory of Belgium, Ringlaan 3, 1180 Brussels, Belgium

J. Vos, G. Raskin, H. Van Winckel

Instituut voor Sterrenkunde, KU Leuven, Celestijnenlaan 200D, 3001 Leuven, Belgium 


\begin{abstract}
Gamma Doradus stars (hereafter $\gamma$ Dor stars) are gravity-mode pulsators of spectral type A or F. Such modes probe the deep stellar interior, offering a detailed fingerprint of their structure. Four-year high-precision space-based Kepler photometry of $\gamma$ Dor stars has become available, allowing us to study these stars with unprecedented detail. We selected, analysed, and characterized a sample of $67 \gamma$ Dor stars for which we have Kepler observations available. For all the targets in the sample we assembled high-resolution spectroscopy to confirm their F-type nature. We found fourteen binaries, among which four single-lined binaries, five double-lined binaries, two triple systems and three binaries with no detected radial velocity variations. We estimated the orbital parameters whenever possible. For the single stars and the single-lined binaries, fundamental parameter values were determined from spectroscopy. We searched for period spacing patterns in the photometric data and identified this diagnostic for 50 of the stars in the sample, 46 of which are single stars or single-lined binaries. We found a strong correlation between the spectroscopic $v \sin i$ and the period spacing values, confirming the influence of rotation on $\gamma$ Dor-type pulsations as predicted by theory. We also found relations between the dominant g-mode frequency, the longest pulsation period detected in series of prograde modes, $v \sin i$, and $\log T_{\text {eff }}$.
\end{abstract}

Subject headings: asteroseismology - stars: variables: general - stars:fundamental parameters stars: oscillations

\title{
1. Introduction
}

One of the most important elements of modern astronomy and astrophysics is the theory of stellar structure and evolution. It has a direct impact on research domains such as the interstellar medium, exoplanets, galactic evolution etc. While the general outline of this theory is nowadays well understood and globally accepted, various aspects such as the interior stellar rotation and the chemical mixing processes inside stars are largely unknown.

A key element of stellar structure and evolution which may vary between different theoretical models is the evolution of the convective core in intermediate-mass $\left(1 M_{\odot} \lesssim M_{*} \lesssim 2 M_{\odot}\right)$ main-sequence stars. This is the transition range from low-mass stars with a radiative core and a convective envelope to high-mass stars with a convective core and a radiative envelope. For these stars the evolution of the convective core throughout the star's core-hydrogen burning phase is dominated by the changes in the local opacity and the

\footnotetext{
${ }^{2}$ Postdoctoral Fellow of the Fund for Scientific Research (FWO), Flanders, Belgium.

${ }^{3}$ Postdoctoral Fellow of the Fund for Scientific Research (FWO), Flanders, Belgium.

${ }^{4} \mathrm{Ph} . \mathrm{D}$. Fellow of the Fund for Scientific Research (FWO), Flanders, Belgium.
} 
energy produced by the $\mathrm{CNO}$ hydrogen burning cycle with respect to the $p p$ chains. Due to the interplay of these physical processes, the convective core can either grow or shrink during the core hydrogen burning phase, depending on the star's initial mass (e.g. Mitalas 1972; Crowe \& Mitalas 1982; Silva Aguirre et al. 2011). The precise transition point between these two regimes in theoretical models not only depends on the star's chemical composition and the internal mixing processes, but also on their numerical treatment (Miglio et al. 2008).

A good way to calibrate and improve upon the existing stellar structure and evolution theories in this mass range, is a detailed asteroseismic study of $\gamma$ Dor pulsators. Gamma Dor stars are slightly more massive (1.4 to $2.5 M_{\odot}$ ) and more luminous than the Sun and exhibit non-radial gravity-mode pulsations (e.g., Kaye et al. 1999). The pulsations, which have a typical period between 0.3 and 3 days and are excited by the convective flux blocking mechanism at the bottom of the convective envelope, probe the internal stellar structure of the stars up to the edge of the convective core (Guzik et al. 2000; Dupret et al. 2005). It was shown by Tassoul (1980) that for a non-rotating star consisting of a convective core surrounded by a chemically homogeneous radiative envelope, pulsations with the same spherical degree $l$ and different radial orders $n$ are equidistantly spaced in the period domain if $l \ll n$. This model was strongly improved upon by Miglio et al. (2008), who showed that a chemical gradient at the edge of the convective core modifies the g-mode resonance cavity, which in turn results in periodic dips in the period spacing pattern, where the depth and the regularity of the dips indicate the steepness and the location of the gradient, respectively. In the case of a growing convective core, there is a chemical discontinuity at the edge of the core, whereas a shrinking core leaves behind a chemical gradient in the near core region. Bouabid et al. (2013) studied the influence of extra mixing processes and stellar rotation. Chemical mixing washes out the $\mu$-gradient, which strongly reduces the prominence of the dips in the period spacing pattern.

Rotation introduces frequency splitting, which in the case of fast rotation results in separated period spacing patterns depending on the value of the azimuthal order $m$. Retrograde mode patterns will typically have an upward slope, while both prograde and zonal mode patterns have a downward slope in the graphical representation of $\Delta P$ versus $P$ (Bouabid et al. 2013). For modes excited with equal amplitude, it is expected that prograde sectoral modes (with $0<m=l$ ) are the easiest to detect. It has been shown that for all other pulsation modes the Coriolis force will trap the pulsation within an equatorial waveguide, which becomes more narrow as the rotational velocity increases. As a result, the geometrical cancellation effects will be more pronounced for these oscillation modes, making it harder to detect them (Townsend 2003).

Thanks to the space-mission Kepler (Koch et al. 2010), we now have photometric observations of four year duration, which is essential for gravity-mode pulsators to enable successful seismic modelling (e.g., Pápics et al. 2014). The advantage of space-based observations is the large number of frequencies which can be resolved, as opposed to only a handful of modes detected in ground-based data of $\gamma$ Dor stars (e.g., Cuypers et al. 2009). Various period spacing patterns for AF-type main-sequence pulsators have meanwhile been reported (e.g., Chapellier et al. 2012; Kurtz et al. 2014; Bedding et al. 2014; Saio et al.|2015), as well as sample compilations and studies of such pulsator classes (e.g. Grigahcène et al. 2010; Uytterhoeven et al. 2011; Balona et al. 2011; Bradley et al. 2015). 
Here, we study the sample of $70 \gamma$ Dor candidates as composed by Tkachenko et al. (2013a) in full depth. For all the stars in this sample, we collected the four-year high-precision Kepler photometry and we gathered high-resolution spectroscopy to make a full observational characterization of the sample. This allows us to do a detailed statistical analysis of the observed properties for this sample of gravity-mode pulsators. In Section 2, we discuss the available data and the applied data reduction methods. In Section 3. we evaluate the $\gamma$ Dor-type nature of the targets and check for binarity. We also list the fundamental parameter values derived from the spectroscopy. We then look for period spacing patterns in the photometric data in Section 4 and check for correlations with the spectroscopic parameter values (Section 5), before coming to our conclusions (Section 6). All our data produces are made publicly available along with this publication.

\section{Observations and data reduction}

For each star in our sample we obtained high-resolution spectra between May and August in 2011, 2013, and 2014 with the HERMES spectrograph $(\mathrm{R}=85000, \lambda=377-900 \mathrm{~nm}$, Raskin et al. (2011) ) at the 1.2-m Mercator telescope (Observatorio del Roque de los Muchachos, La Palma, Canary Islands, Spain). The total number of spectra and observation times are listed per star in the appendix in Table A1.

The obtained spectra were reduced with the most recent version of the HERMES pipeline (release 5 ) and the individual spectra were subsequently normalised in two steps by fitting a cubic and linear spline through the flux continuum respectively, as described by Pápics et al. (2012). Outliers in the spectra resulting from cosmic hits were identified by comparing the local flux with the median value in the surrounding spectral region (of 40 pixels). If the difference exceeded $5 \sigma$, where $\sigma$ is determined as the local signal-tonoise $(\mathrm{S} / \mathrm{N})$ ratio in the spectrum, the flux point was replaced by the median value.

In addition to the spectroscopic observations, all the stars in our sample have been observed by the Kepler mission with long cadence (i.e. with an integration time of 29.4 minutes). For the majority of stars, data are available for all eighteen quarters (Q0-Q17), while the shortest obtained light curves cover eight quarters. The available quarters are also listed for each star in TableA1

Because the standard light curve extraction algorithm, provided by the Mikulski Archive for Space Telescopes (MAST), is known to result in residual instrumental signal in the low frequency region (Debosscher et al. 2013; Tkachenko et al. 2013a), we apply the light curve extraction algorithm developed by one of us (S.B.). The code is based on the use of customised masks, which allows us to include the signal from more pixels, thereby reducing the influence of instrumental trends. The light curves of each individual quarter were subsequently detrended by changing the scale of the extracted light curves to magnitude and subtracting a $1^{\text {st }}$ or $2^{\text {nd }}$-order polynomial, and concatenated. The outliers of the light curves were removed manually. We then extracted the frequencies from the reduced light curves by an iterative prewhitening method based on the Lomb-Scargle periodogram, as described in detail by Van Reeth et al. (2015). Observed oscillation frequencies above the Nyquist frequency were evaluated by comparing their amplitudes with those of possible aliases. Following Murphy et al. (2013), the frequency with the highest amplitude was retained. The errors 
of the frequency values were determined using the method by Schwarzenberg-Czerny (2003), which is based on the statistical errors resulting from a non-linear least-squares fit corrected for the correlated nature of the data.

\section{Spectroscopic analysis}

Visual inspection of the available spectra revealed that two of the targets, KIC 5772452 and KIC 7516703 , are in fact rotationally modulated K-type stars. They have therefore been discarded for the rest of this paper. In addition, one of the stars in the sample, KIC 10080943, had already been identified as a double-lined binary by Tkachenko et al. (2013a). This star has been monitored extensively in spectroscopy meanwhile and reveals tidally-excited as well as free oscillations. Given that some of its modes are of different origin than those of the other sample stars, it will be discussed separately (Schmid et al, in preparation). This leaves us with 67 stars for the current study.

\subsection{Binarity}

In a first step in our data analysis we checked the targets in our sample for binarity. We applied the improved least-squares deconvolution (LSD) algorithm, as described by Tkachenko et al. (2013c), to the individual spectra of each star using a line mask with typical parameter values for a $\gamma$ Dor star $\left(T_{\text {eff }}=\right.$ $\left.7000 \mathrm{~K} ; \log g=4.0 ;[M / H]=0.0 ; \zeta=2 \mathrm{~km} \mathrm{~s}^{-1}\right)$. The line mask was computed with the spectrum synthesis synthV code (Tsymbal 1996), which makes use of a spectral line list (obtained from the VALD database, Kupka et al. (1999)) and a predefined grid of atmospheric models (computed with the LLmodels code, Shulyak et al. (2004)). This resulted in high $\mathrm{S} / \mathrm{N}$ average line profiles which were checked for the presence of a signal from a possible companion. This resulted in the detection of four single-lined binaries (KIC 6468146, KIC 7867348, KIC 10467146, KIC 11754232), four double-lined binaries (KIC 3222854, KIC 3952623, KIC 6367159, KIC 8693972), two triple systems (KIC 6467639, KIC 6778063) and three binaries for which we have spectral contributions from both components, but no detected radial-velocity variations. This is also listed in Table A2

The radial velocities of the different components of a binary were obtained by taking the center of gravity of their LSD profile 1 For some of the detected single- and double-lined binaries we have obtained a sufficient number of observations to cover the binary orbit, allowing us to obtain an (order of magnitude) estimate of the orbital period $P$, as shown in Table1 For KIC 3952623, KIC 7867348, and KIC 11754232 these values were sufficiently accurate to allow us to determine values for the remaining spectroscopic orbital parameters (eccentricity $e$, the angle $\omega$ between the ascending node and the periapsis, the time $T_{0}$ of periastron passage, the system velocity $\gamma$, velocity amplitudes $K_{j}$ and the projected semi-major axes $a_{j} \sin i$ )

\footnotetext{
${ }^{1}$ These are available from https://fys. kuleuven.be/ster/Projects/gamma-doradus
} 
Table 1. The obtained parameter values and their respective error margins for several of the binary systems in our sample. For KIC 3952623, KIC 7867348 and KIC 11754232 we were able to obtain an orbital solution.

\begin{tabular}{|c|c|c|c|c|c|c|c|}
\hline & KIC 3222854 & & KIC 3952623 & & KIC 7836348 & KIC 8693972 & KIC 11754232 \\
\hline method & spectroscopy & spectroscopy & FM method & PM method & spectroscopy & spectroscopy & spectroscopy \\
\hline$P$ [days] & $\gtrsim 100$ & 19.515 & 19.5 & 19.526 & 10.0132 & $\sim 60,90,120$ or 180 & 214.9 \\
\hline$\sigma_{P}$ & $\ldots$ & 0.006 & 0.2 & 0.001 & 0.0006 & $\cdots$ & 0.6 \\
\hline$e$ & $\ldots$ & 0.17 & 0 & 0.191 & 0.23 & $\cdots$ & 0.55 \\
\hline$\sigma_{e}$ & $\cdots$ & 0.04 & $\cdots$ & 0.001 & 0.03 & $\cdots$ & 0.01 \\
\hline$\omega[2 \pi]$ & $\cdots$ & 1.65 & $\cdots$ & 1.5481 & 1.05 & $\cdots$ & 4.52 \\
\hline$\sigma_{\omega}$ & $\cdots$ & 0.1 & $\cdots$ & 0.0006 & 0.11 & $\cdots$ & 0.03 \\
\hline$T_{0}[\mathrm{BJD}]$ & $\cdots$ & 2456442.8 & $\cdots$ & $\cdots$ & 2455685.96 & $\cdots$ & 2456417.23 \\
\hline$\sigma_{T_{0}}$ & $\ldots$ & 0.3 & $\cdots$ & $\cdots$ & 0.15 & $\cdots$ & 1.38 \\
\hline$K_{1}\left[k^{-1} \mathrm{~s}^{-1}\right]$ & $\cdots$ & 30 & 30.08 & 19.36 & 9.3 & $\cdots$ & 17.8 \\
\hline$\sigma_{K_{1}}$ & $\cdots$ & 0.8 & $\cdots$ & 0.04 & 0.2 & $\cdots$ & 0.2 \\
\hline$K_{2}\left[k^{-1} ~ s^{-1}\right]$ & $\cdots$ & 52.5 & $\cdots$ & $\cdots$ & $\cdots$ & $\cdots$ & $\cdots$ \\
\hline$\sigma_{K_{2}}$ & $\cdots$ & 1.4 & $\cdots$ & $\cdots$ & $\cdots$ & $\cdots$ & $\cdots$ \\
\hline$a_{1} \sin i[\mathrm{AU}]$ & $\cdots$ & 0.053 & 0.054 & $\cdots$ & 0.0083 & $\cdots$ & 0.294 \\
\hline$\sigma_{a_{1} \sin i}$ & $\cdots$ & 0.001 & $\cdots$ & $\cdots$ & 0.0002 & $\cdots$ & 0.004 \\
\hline$a_{2} \sin i[\mathrm{AU}]$ & $\cdots$ & 0.093 & $\cdots$ & $\cdots$ & $\cdots$ & $\cdots$ & $\cdots$ \\
\hline$\sigma_{a_{2} \sin i}$ & $\cdots$ & 0.003 & $\cdots$ & $\cdots$ & $\cdots$ & $\cdots$ & $\cdots$ \\
\hline$\gamma\left[k m s^{-1}\right]$ & -39.5 & -15.7 & $\ldots$ & $\ldots$ & -26 & -5.9 & 13.5 \\
\hline$\sigma_{\gamma}$ & 1.7 & 2.5 & $\cdots$ & $\cdots$ & 0.2 & 3.9 & 0.4 \\
\hline
\end{tabular}


as well, which are again listed in Table 1 The radial velocity variations of the two triple systems proved to be complicated to unravel. Here a more detailed analysis is required, which is outside the scope of this paper.

We also applied the frequency modulation (FM) method (Shibahashi \& Kurtz 2012) and the phase modulation (PM) method (Murphy et al. 2014) to the pulsation frequencies extracted from the Kepler light curves. As Murphy et al. (2014) pointed out, the FM method is most sensitive for the detection of relatively short-period binaries, the PM method is more likely to be successful for the detection of long-period binaries and lower-frequency pulsations. In the case of KIC 3952623, we were able to confirm the spectroscopic results with both the FM and PM methods, as shown in Table1 though the PM method leads to an underestimate of the velocity amplitude due to the relatively short orbital period. Neither method provided a satisfactory result for the other observed binaries. There are several possible explanations. Firstly, an important requirement for these methods is that $P_{\text {puls }} \ll P_{\text {orbit }}$. The typical $\gamma$ Dor pulsation period has a value between 0.3 and 3 days, so that often the difference between $P_{\text {puls }}$ and $P_{\text {orbit }}$ is too small. Secondly, as we will see in Section 4 we often observe period spacing patterns with a value between 0.001 and $0.035 \mathrm{~d}$, depending on the rotation velocity and the spherical degree $l$ of the mode. The corresponding spacings in the frequency domain are close to the ones we would obtain for a binary orbit above $20 \mathrm{~d}$. Aside from KIC 3952623, there are indications of the presence of the expected signal for KIC 11754232, but unfortunately we have only eight quarters of data available for this star, which prevented a reliable analysis.

Out of the fourteen targets that we found to be binaries, seven are identified as $\gamma$ Dor $/ \delta$ Sct hybrid pulsators (out of eleven hybrids in the studied sample of 69 targets). While this could mean that several of these binary systems consist of a $\gamma$ Dor and a $\delta$ Sct star, we cannot exclude the possibility that one (or both) of the components are hybrid pulsators. Indeed, three hybrids are identified as single-lined binaries, and from the application of the FM method to KIC 3952623 we conclude that the studied $\delta$ Sct pulsations belong to the brightest component, which seems to indicate that the primary is a hybrid, considering the relatively high amplitudes of the $\gamma$ Dor pulsations.

\subsection{Fundamental parameters}

In the second part of the spectroscopic analysis, we focused on the stars which have been classified as either single stars or single-lined binaries. The spectra of these stars were corrected for any radial velocity variations (in the case of the single-lined binaries) and merged. This resulted in a single spectrum for each star, which we subsequently used to determine the fundamental spectroscopic parameter values. The advantage is that such a combined spectrum has a higher $\mathrm{S} / \mathrm{N}$ ratio and that pulsation line profile variations, which might otherwise skew the spectral analysis, are largely averaged out.

We use the Grid Search in Stellar Parameters (GSSP) code for the spectral analysis (Lehmann et al. 2011; Tkachenko et al. 2012). The values of the temperature $T_{\text {eff }}$, surface gravity $\log g$, metallicity $[M / H]$, microturbulence $\zeta$ and rotational velocity $v \sin i$ are determined from a $\chi^{2}$-minimization for the observed spectra with respect to synthetic spectra within a relatively large wavelength range. The synthetic spectra 
are computed for different values of the listed parameters using a combination of the synthV code (Tsymbal 1996), the LLmodels code (Shulyak et al.2004) and information obtained from the VALD database (Kupka et al. 1999), i.e. similar to how the LSD line mask was computed. In this study we took the spectral range between $4700 \AA$ and $5800 \AA$, which includes the $H_{\beta}$-line. For a typical $\gamma$ Dor star, the Balmer lines are mostly sensitive to $T_{\text {eff }}$ (e.g. Gray 1992). By including the $H_{\beta}$-line into the spectral fits, we were able to put strong constraints on the values of $T_{\text {eff }}$.

The obtained parameter values are listed in Table $\mathrm{A3}$ and some sample distributions are illustrated in Fig.1 As we can see, $T_{\text {eff }}$ typically has a value near $7100 \mathrm{~K}$, while $\log g$ is close to 4.1 . The metallicity, $[M / H]$, is on average slightly higher than the solar metallicity, while $\zeta$ typically has a value between 2 and $3.5 \mathrm{~km} \mathrm{~s}^{-1}$. These are in close agreement with the parameters we had chosen for the LSD line mask in Section 3.1. The metallicity values previously computed by Tkachenko et al. (2013a) are on average slightly below the solar value, because $\zeta$ was kept fixed at $2 \mathrm{~km} \mathrm{~s}^{-1}$ in that study, whereas we included it as a free parameter. We also find that most $\gamma$ Dor stars in our sample are slow to moderate rotators compared to their break-up velocity $v_{c}=\sqrt{2 G M_{*} / 3 R_{*}}$, where $M_{*}$ and $R_{*}$ are the stellar mass and radius. For a $1.6 M_{\odot}$ star with a $1.9 R_{\odot}$ radius we find $v_{c}=327 \mathrm{~km} \mathrm{~s}^{-1}$. Typical values for $\gamma$ Dor stars are all on the order of $300 \mathrm{~km} \mathrm{~s}^{-1}$.

A comparison of the spectroscopically derived parameters values of $T_{\text {eff }}, \log g$ and $[M / H]$ with the values found in the KIC catalogue, which are derived from SED fitting, is shown in Fig. 2 It is found that, apart from a few outliers, the $T_{\text {eff }}$ values in the KIC catalogue are reasonably reliable, while the deviations of the surface gravity and metallicity values seem to be correlated to the actual parameter values. This relation is similar to the one found by Tkachenko et al. (2013b).

\section{Period spacing patterns in the Kepler photometry}

Having confirmed the $\gamma$ Dor nature for the vast majority of the targets in our sample and having identified the binaries, we used the extracted pulsation frequencies to look for period spacings by means of the methodology described in Van Reeth et al. (2015). This resulted in the detection of period spacings for 45 of the 67 studied targets. These are listed in Tables A2 and A4 The found spacing patterns are all shown in Appendix A A selection can also be found in Figs. 3 to 6

Most of the period spacing patterns have a clear slope. This has been observed before Bedding et al. 2014; Van Reeth et al. 2015) and is a consequence of frequency shifts due to rotation. Interestingly, most of the detected period spacing patterns seem to be complementary to the ones presented by Bedding et al. (2014). These authors considered slowly rotating stars without spectroscopic confirmation that it actually concerns F-type stars, while our method is also suitable to detect the period spacing patterns of the moderate to fast rotating stars in our sample. While this difference is clearly visible in the increasingly strong downward slopes of many of the detected patterns, there are also several detected spacing series with an upward slope.

As discussed in Section 1 the period spacing patterns with a downward slope are either prograde or 

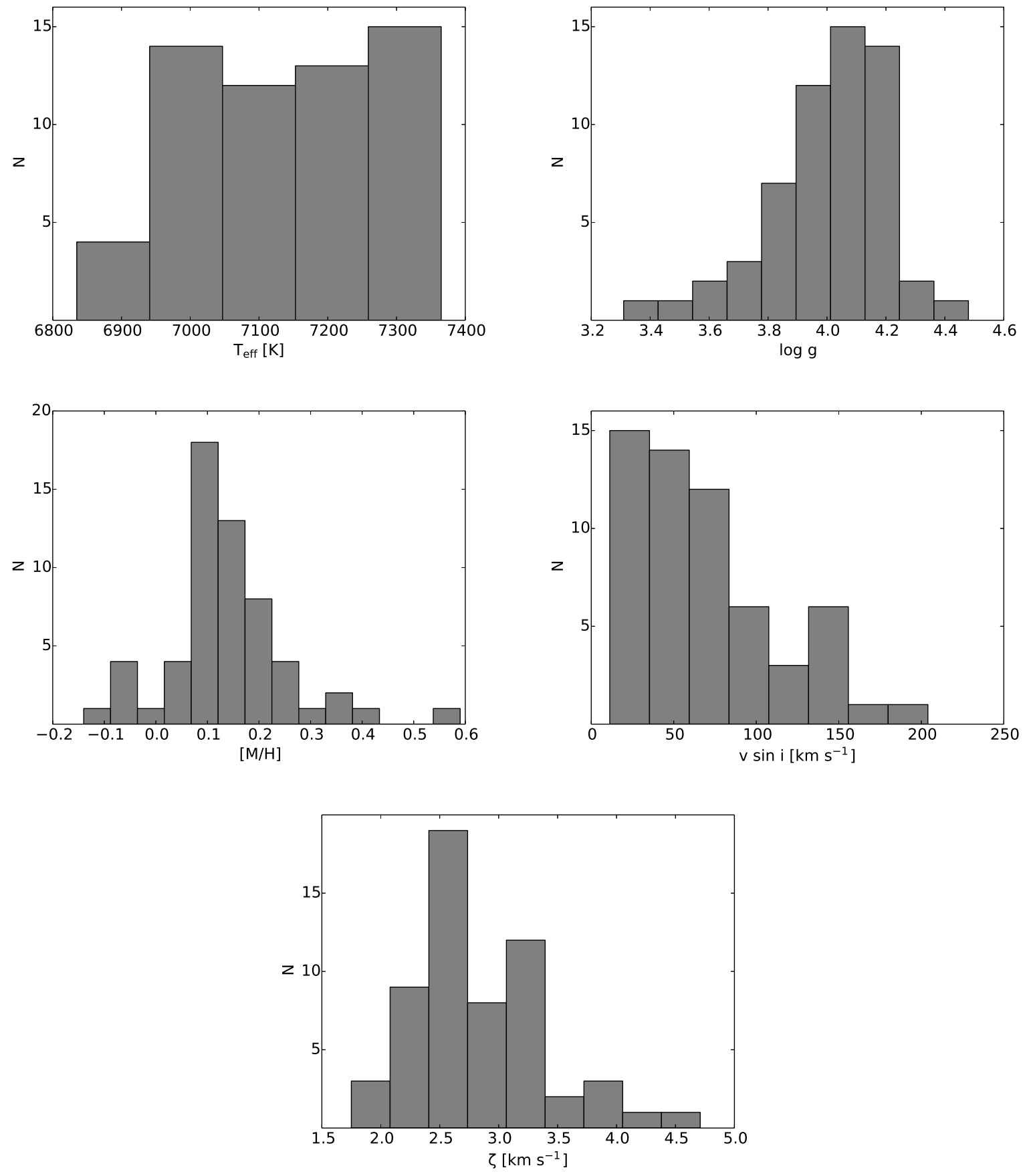

Fig. 1.- Histograms representing the distribution of the atmospheric parameter values obtained for the single stars and the single-lined binaries in our sample. 
zonal modes. Given that zonal modes are expected to be more susceptible to trapping in an equatorial waveguide under the influence of the Coriolis force (Townsend 2003), we expect most, if not all, of the detected modes to be prograde. According to theory, spacing series with an upward slope correspond to retrograde modes that are shifted in the frequency domain because of the stellar rotation. It is expected that the average period spacing value of retrograde modes increases for higher rotation rates Bouabid et al. 2013), as found for KIC 11145123 (Kurtz et al. 2014), KIC 9244992 (Saio et al. 2015) and KIC 9751996 (see Fig.3). Nevertheless, for several stars, such as e.g. KIC 8375138 (see Fig.44), the observed mean period spacing value for the series with the upward slope is smaller than it would be if the star did not rotate. The reason is that for these stars, the rotation frequency is so high the retrograde modes are actually shifted below zero in the inertial reference frame. When observed, pulsation frequencies are assumed to be positive, so that when the retrograde modes are observed, we end up with a spacing pattern such as the one seen in Fig.4

While Bouabid et al. (2013) already mentioned that this might occur, we find this to be fairly common since no less than ten stars with a detected period spacing pattern are fast rotators exhibiting both prograde and retrograde modes.

For six stars in our sample, such as KIC 11668783 illustrated in Fig.6, we find a period spacing pattern connected with retrograde modes only, all of which having similar characteristic amplitude patterns in the sense that the mode amplitude grows as the pulsation period increases and drops sharply (see Figs. 4 and 57. The amplitude comb of KIC 8375138 as seen in Fig. 4 reveals dips in the amplitudes in an otherwise monotonically growing period series.

Even though many of the observed periods spacing patterns are reasonably smooth, there are also several patterns which show regular dips. This is particularly visible for the pulsation spacing pattern of KIC 2710594, shown in Fig.5 Finally, several of the stars for which we find period spacing patterns are hybrid $\gamma$ Dor/ $\delta$ Sct stars, most notably KIC 8645874, KIC 9751996 and KIC 11754232 (listed in Table A2). Not only are these single stars or single-lined binaries (KIC 11754232), they are also slow rotators and have a relatively rich pulsation spectrum in the $\delta$ Sct regime, making them interesting targets to probe the interior stellar rotation profile, as done in Kurtz et al. (2014) and Saio et al. (2015).

\section{Statistical analysis of the sample properties}

Among the 45 stars for which we found period spacing patterns, 38 are single stars and three are singlelined binaries. We have therefore 41 stars for which we have both spectroscopic and pulsational parameters based on period spacings, allowing us to look for possible correlations between their properties and compare those qualitatively with the relations predicted by theory.

In order to properly take into account the characteristics of the period spacing patterns in the analysis, we limited ourselves to the patterns with a downward slope, because these are by far the most frequently detected. We derived various quantities from them, as illustrated in Fig. 7 The mean pulsation period of the g-modes that are part of the found series is denoted as $\langle P\rangle$, while $\langle\Delta P\rangle$ is the mean spacing value of the pattern. In addition, we have the mean derivative $\mathrm{d}(\Delta P) / \mathrm{d} P$ of the pattern, which is actually computed 

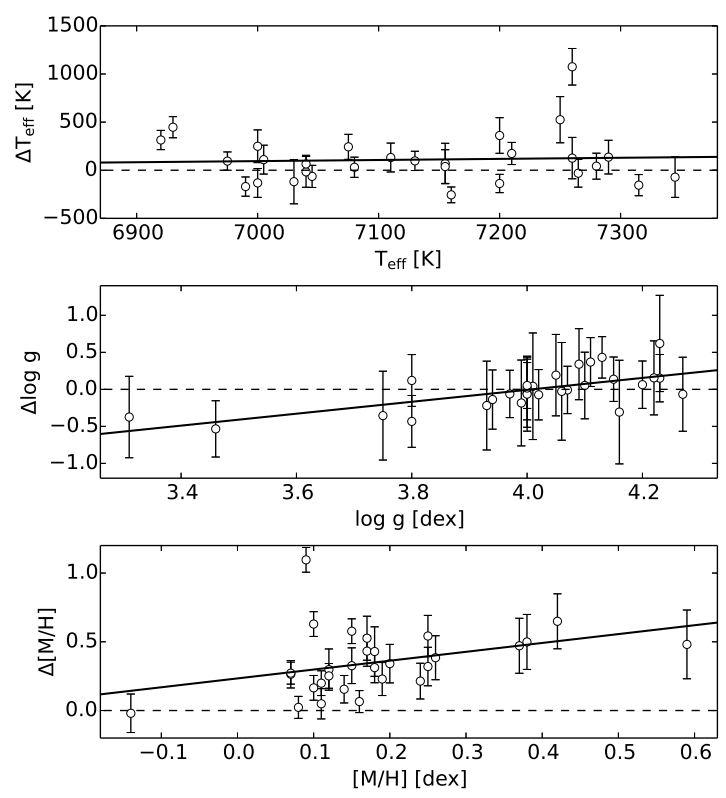

Fig. 2.- The differences between the spectroscopically derived values and the KIC values of the fundamental parameters $T_{\text {eff }}$ (top), $\log g$ (middle) and $[M / H]$ (bottom), as a function of the spectroscopic values. Linear fits of the relations are represented by the solid black lines.
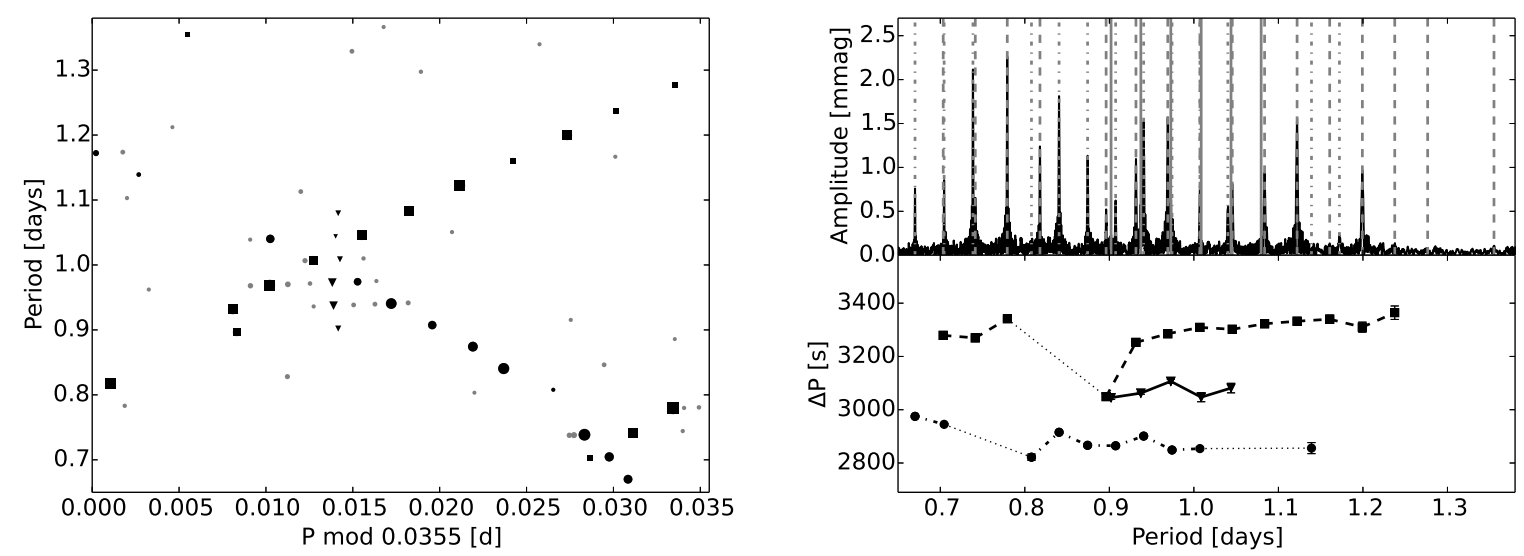

Fig. 3.- The period spacing patterns of the slowly rotating star KIC 9751996. Left: the pulsation series in a period échelle diagram. The prograde (black circles), zonal (black triangles) and retrograde (black squares) dipole modes are marked separately, while the other pulsation modes are shown in grey. Top right: the prograde (dash-dot lines), zonal (full lines) and retrograde (dashed lines) modes in the Fourier spectrum. Bottom right: the period spacing patterns, using the same symbols as before. The dotted lines indicate missing frequencies. 

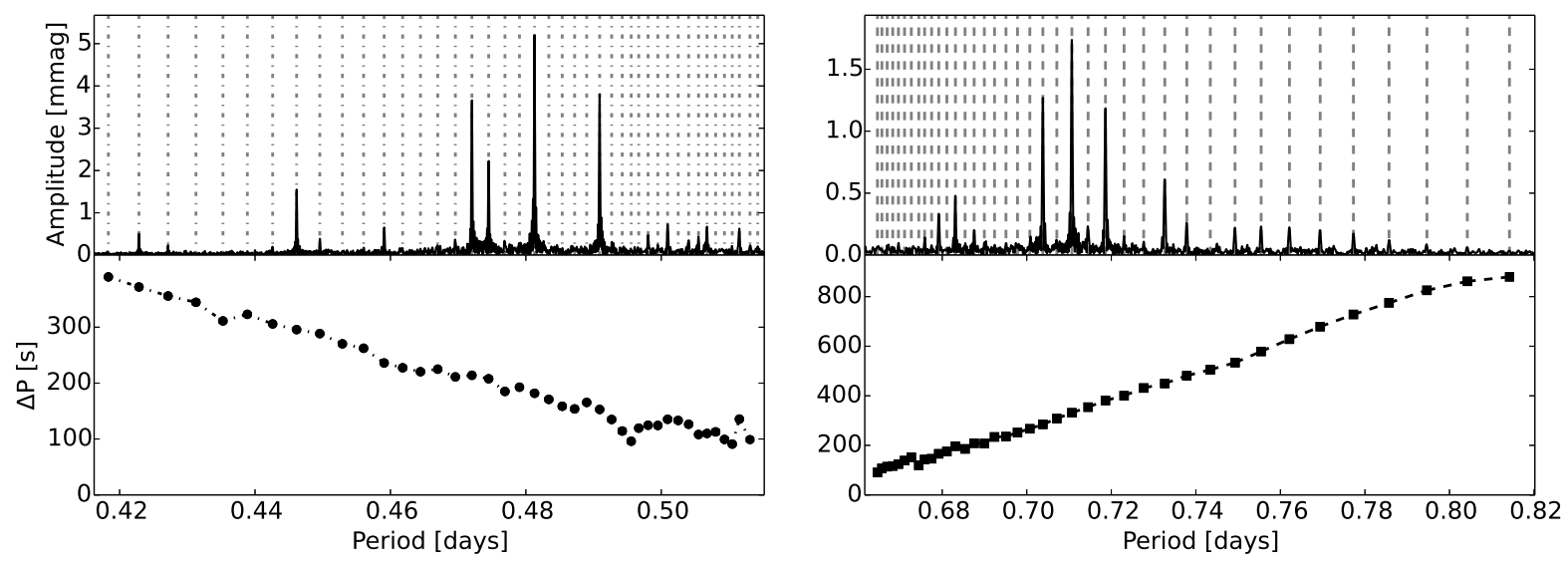

Fig. 4. - The prograde (left) and retrograde (right) period spacing patterns of KIC 8375138. The used symbols are the same as in Fig. 3 .
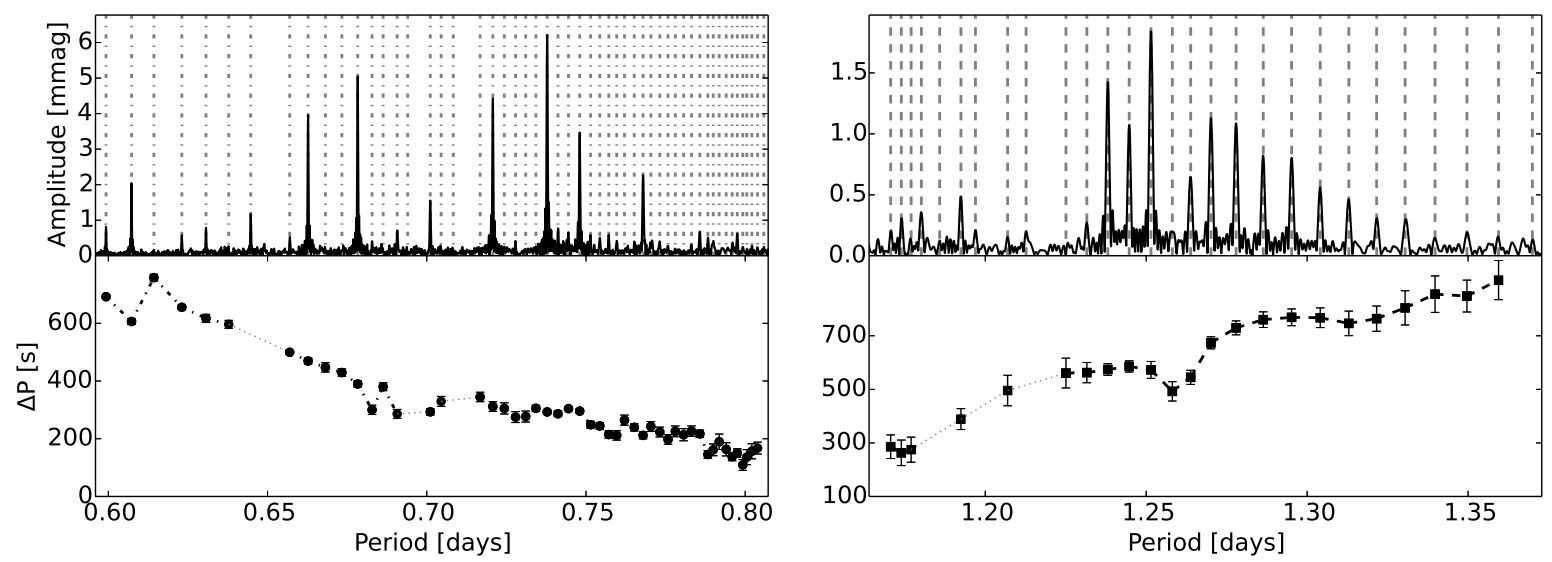

Fig. 5.- The prograde (left) and retrograde (right) period spacing patterns of KIC 2710594. The used symbols are the same as in Fig. 3. 
from a fitted $2^{\text {nd }}$ order polynomial (shown in red in Fig.7) as this allows us to avoid the influence of any irregularities or dips in the spacing series.

We also took into account the parameter $\Delta_{\text {res }}$, which is a measure of the residuals of the polynomial fit to the period spacing series as a proxy of the smoothness of the found pattern. The parameter $P_{\max }$, on the other hand, is the longest detected pulsation period of the downward spacing pattern. We here consider it to be indicative of the cut-off frequency for which the radiative damping of the pulsations in the g-mode cavity becomes dominant over the excitation (Dupret et al. 2005; Bouabid et al. 2013). In general, it is the interplay between the non-adiabatic excitation and damping, taking into account advective damping due to wave leakage caused by the Coriolis force, that determines whether a mode will be excited to detectable amplitude or not (Townsend 2000). The observed quantity $P_{\max }$ is therefore not only of interest to deduce the boundaries of the $\gamma$ Dor instability strip, but also to derive the physical conditions that determine the delicate balance between damping and excitation of the modes.

The other included parameters in our analysis are the frequency $f_{g}$ and the amplitude $A_{g}$ of the dominant g-mode as well as the obtained spectroscopic stellar parameters. While it would have been valuable to also include the frequency $f_{p}$ and the amplitude $A_{p}$ of the $\delta$ Sct p-mode with the highest amplitude or a proxy for the acoustic cutoff frequency for the hybrid $\gamma$ Dor $/ \delta$ Sct stars, there are not enough hybrids in the sample for a meaningful statistical analysis based on these quantities. Similarly, the number of sample stars with retrograde modes is also too small for such a study.

We looked for linear relations between the different established parameters, where we took the logarithm for those quantities whose range is two or more orders of magnitude. These are the twelve parameters listed in Tables A3 and A4 for the 41 stars with a downward period spacing pattern. We performed several multivariate analyses, each time choosing one of the available parameters as the dependent variable $Y$ and taking the others as covariates $X_{i}$. In order to make sure that there was no offset in the parameter relation, we subtracted the mean values of all the parameters prior to the regression analysis. The latter was based on forward selection and backward elimination (e.g., Aerts et al. 2014, for a similar application). With the forward selection method, we add covariates one at a time as long as their addition implies a significantly better model fit, starting with the one showing the strongest correlation with the chosen dependent variable. In the case of the backward elimination, we start with all covariates under study and remove those showing insignificant correlation with the dependent variable. The significance tests for each individual covariate were done using a $t$-test, while the evaluation of each step of the fit was based on the coefficient of determination $R^{2}$, i.e., the fraction of the variance of the dependent variable explained by the model. A summary of the multiple regression results is provided in Table 2 .

The clearest result from these analyses is the strong correlation between the stellar rotation and the found period spacing, as predicted by theory (Bouabid et al. 2013) and already discussed in Sections 1 and 4 The faster a star is rotating, the larger the rotational shifts of the pulsation frequencies, which for prograde modes translates into a period spacing pattern with a steeper downward slope and consisting of more closely spaced, shorter pulsation periods. The bivariate relation between the mean period spacing and $\log (v \sin i)$ is shown in Fig. 8 

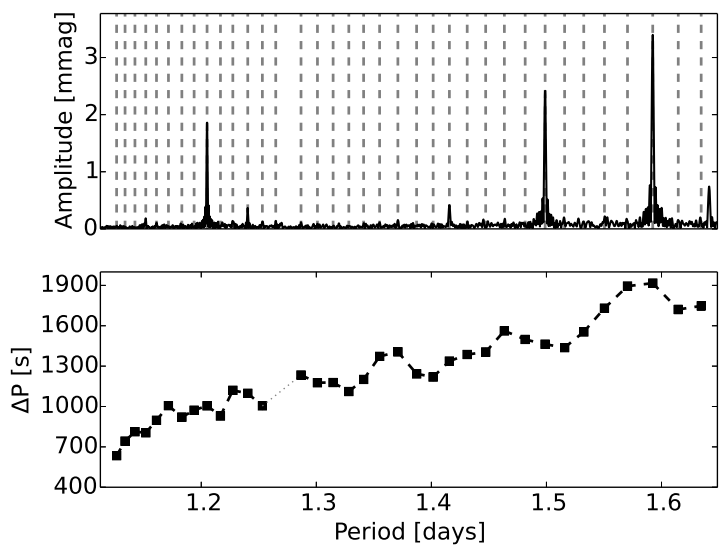

Fig. 6. - The retrograde period spacing patterns of KIC 11668783. The used symbols are the same as in Fig. 3 .

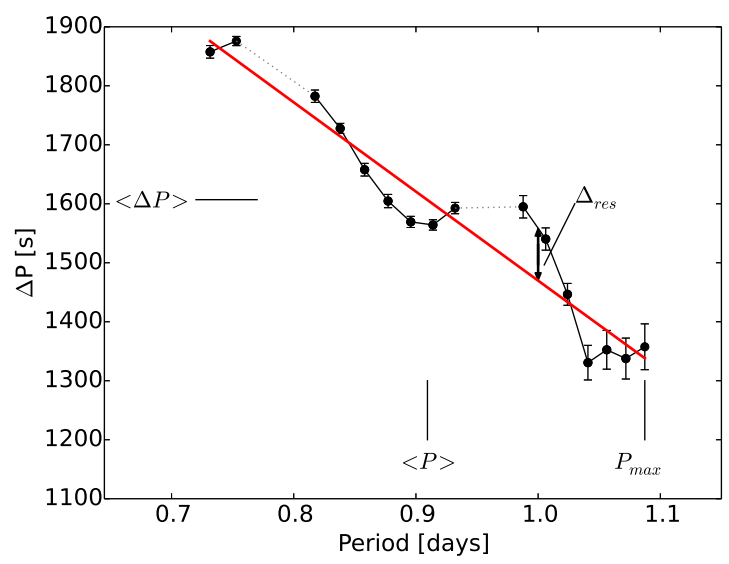

Fig. 7.- Illustration of some of the photometric parameters from the period spacing patterns (shown here for KIC 10224094) for the statistical analysis: $\langle P\rangle$ is the mean pulsation period, $P_{\max }$ is the longest pulsation period, $\langle\Delta P\rangle$ is the mean spacing, and $\left\langle\frac{\mathrm{d} \Delta P}{\mathrm{~d} P}\right\rangle$ is the mean derivative (computed from the polynomial fit, here shown in red). Finally, $\Delta_{\text {res }}$ is a measure of the difference between the polynomial fit and the period spacing pattern. 
Secondly, we found that the value of dominant mode frequency, $f_{g}$, is strongly correlated with the values of $v \sin i$ and $\log T_{\text {eff }}$, as shown in Fig.9 As listed in Table_ the $R^{2}$-value we obtain for the bivariate model based on $f_{g}$ and $v \sin i$ is 0.468 . When we include $\log T_{\text {eff }}$ as well, the value of $R^{2}$ increases to 0.665 for the trivariate model fit, given by

$$
f_{g}=30.9(6.6) \cdot \log T_{\mathrm{eff}}+0.0103(0.0013) \cdot v \sin i-118.23(0.32) \text {, }
$$

where the frequency $f_{g}$ is expressed in $d^{-1}, T_{\text {eff }}$ in Kelvin and $v \sin i$ in $\mathrm{km} \mathrm{s}^{-1}$. The relation was found for both the forward and the backward modelling and is clearly appropriate. However, there are two unknowns causing variance in this trivariate model that we cannot take into account at present: the inclination angle $i$ of the star and the spherical degree $l$ of the pulsation modes. This may explain some of the scatter in Fig. 9

It is noteworthy that the trend between $f_{g}$ and $\log T_{\text {eff }}$ is opposite to the one found for B-type gmode pulsators, which do not have an outer convective envelope and are, in general, slower rotators than the sample we studied here (De Cat \& Aerts 2002; Aerts et al. 2014). However, the two correlations are not directly comparable because the instability strip of SPB stars is much more extended along the main sequence than the $\gamma$ Dor instability strip. The latter is confined to a much more narrow $\log T_{\text {eff }}$ range than the SPB instability region. Therefore the main contributor to the above mentioned trend for $\gamma$ Dor stars, is the stellar radius, whereas this is the initial mass for SPB pulsators. 
Table 2. Results of the multiple linear regression. We list the coefficients of the covariates for the different model fits as well as the $p$-values (obtained from a $t$-test) and the $R^{2}$ values.

\begin{tabular}{|c|c|c|c|c|c|c|}
\hline \multirow[t]{2}{*}{ Explanatory variables } & \multicolumn{3}{|c|}{ Separate models } & \multicolumn{3}{|c|}{ Multivariate models } \\
\hline & Estimate $(\sigma)$ & $p$-value & $R^{2}$ & Estimate $(\sigma)$ & $p$-value & $R^{2}$ \\
\hline & \multicolumn{6}{|c|}{ Models for the dependent variable $\left\langle\frac{\mathrm{d} \Delta P}{\mathrm{~d} P}\right\rangle$} \\
\hline \multirow[t]{2}{*}{$\log (v \sin i)$} & $-0.032(0.004)$ & 0.025 & 0.606 & $\cdots$ & $\cdots$ & $\cdots$ \\
\hline & \multicolumn{6}{|c|}{ Models for the dependent variable $\langle P\rangle$} \\
\hline Intercept & $\cdots$ & $\cdots$ & $\cdots$ & 77.09 & $\cdots$ & \\
\hline $\log (v \sin i)$ & $-0.45(0.10)$ & 0.0001 & 0.350 & $-0.52(0.09)$ & $<0.0001$ & \\
\hline \multirow[t]{2}{*}{$\log T_{\text {eff }}$} & $-5.8(4.2)$ & 0.313 & 0.050 & $-10.4(3.2)$ & 0.0346 & \\
\hline & \multicolumn{6}{|c|}{ Models for the dependent variable $\langle\Delta P\rangle$} \\
\hline \multirow[t]{2}{*}{$\log (v \sin i)$} & $-0.024(0.002)$ & $<0.0001$ & 0.796 & $\cdots$ & $\ldots$ & $\ldots$ \\
\hline & \multicolumn{6}{|c|}{ Models for the dependent variable $f_{g}$} \\
\hline Intercept & $\cdots$ & $\cdots$ & $\cdots$ & $-118.23(0.32)$ & $\cdots$ & \\
\hline$v \sin i$ & $0.0093(0.0016)$ & $<0.0001$ & 0.468 & $0.0103(0.0013)$ & $<0.0001$ & \\
\hline \multirow[t]{2}{*}{$\log T_{\text {eff }}$} & $22.1(10.5)$ & 0.142 & 0.104 & $30.9(6.6)$ & 0.0047 & \multirow{2}{*}{0.665} \\
\hline & & & & & & \\
\hline$\zeta$ & $0.61(0.14)$ & $<0.0001$ & 0.346 & $\cdots$ & $\cdots$ & $\cdots$ \\
\hline \multirow[t]{2}{*}{$\langle P\rangle$} & $-1.93(0.28)$ & $<0.0001$ & 0.549 & $\cdots$ & $\cdots$ & $\cdots$ \\
\hline & \multicolumn{6}{|c|}{ Models for the dependent variable $P_{\max }$} \\
\hline Intercept & $\cdots$ & $\ldots$ & $\ldots$ & $39.50(0.14)$ & $\ldots$ & \\
\hline$v \sin i$ & $-0.0060(0.0006)$ & $<0.0001$ & 0.703 & $-0.0064(0.0006)$ & $<0.0001$ & \\
\hline \multirow[t]{2}{*}{$\log T_{\text {eff }}$} & $-4.4(5.9)$ & 0.550 & 0.015 & $-10.0(2.9)$ & 0.0292 & \\
\hline & & & & & & 0.773 \\
\hline$\zeta$ & $-0.33(0.07)$ & 0.0079 & 0.358 & $\ldots$ & $\ldots$ & $\ldots$ \\
\hline
\end{tabular}


Table 2-Continued

\begin{tabular}{|c|c|c|c|c|c|c|}
\hline \multirow[t]{2}{*}{ Explanatory variables } & \multicolumn{3}{|c|}{ Separate models } & \multicolumn{3}{|c|}{ Multivariate models } \\
\hline & Estimate $(\sigma)$ & $p$-value & $R^{2}$ & Estimate $(\sigma)$ & $p$-value & $R^{2}$ \\
\hline$\langle P\rangle$ & $1.31(0.08)$ & $<0.0001$ & 0.876 & $\cdots$ & $\cdots$ & $\cdots$ \\
\hline$\left\langle\frac{\mathrm{d} \Delta P}{\mathrm{~d} P}\right\rangle$ & $18.4(2.8)$ & 0.0130 & 0.526 & $\ldots$ & $\ldots$ & $\cdots$ \\
\hline$f_{g}$ & $-0.38(0.06)$ & $<0.0001$ & 0.505 & $\cdots$ & $\cdots$ & $\cdots$ \\
\hline
\end{tabular}


We also found a bivariate relation between $f_{g}$ and $\zeta$. This is not surprising because the overall pulsational line broadening, which is dominated by the mode with frequency $f_{g}$, is captured by $\zeta$. Indeed, we could not perform line-profile modelling due to the individual pulsation modes as it would require timeresolved spectroscopy covering the phases of each and every g-mode (e.g., Aerts et al. 2010, Chapter 6). Hence, the overall pulsational broadening is included in the simple broadening function parametrized with $\zeta$ (Cf. Morel et al. 2006).

Finally, we also found a bivariate relation between $f_{g}$ and $\langle P\rangle$, which indicates that the period spacing patterns are typically found in frequency regions around the dominant g-mode frequency.

The correlations between $f_{g}$ and the other variables also apply to $P_{\max }$ to almost the same extent, as can be seen in Table 2 and as illustrated in Fig.10. The main difference is that the correlation between $P_{\max }$ and $v \sin i$ is stronger than between $f_{g}$ and $v \sin i$, while the correlation with $\log T_{\text {eff }}$ is weaker. Thus, rotation seems to have a larger impact on the period $P_{\max }$ than on the frequency of the dominant mode.

\section{Discussion and conclusions}

This paper offers an observational asteroseismic analysis of 67 of the $\gamma$ Dor stars in the sample presented by Tkachenko et al. (2013a). Thanks to the available combination of Kepler photometry and ground-based high-resolution spectroscopy, we were able to carry out a detailed statistical analysis. We found thirteen of the 67 stars to be members of a binary system. We computed the stellar parameters for all 67 stars in the sample and found them to be consistent with the typical values expected for $\gamma$ Dor stars. Most of the stars in our sample are slow to moderate rotators with respect to their breakup velocity.

For 45 stars in the sample we were able to detect period spacings using the methodology described by Van Reeth et al. (2015). The found spacing patterns are in line with theoretical expectations (Miglio et al. 2008; Bouabid et al. 2013). For several of the stars we detected periodic dips in the period spacing versus period relations, as predicted by Miglio et al. (2008). These dips are caused by the presence of a chemical gradient near the edge of the convective core. For many of the sample stars, the period spacing patterns are smooth, pointing either to extremely young stars or, more likely, to the presence of mixing processes inside the star that have washed out the chemical gradient. A priori one could expect to see a correlation between the smoothness of the period spacing patterns and the observed rotation velocity. In this paper, such a correlation would be reflected between the values of $\Delta_{\text {res }}$ and $v \sin i$, but we could not find a significant relation between the two parameters. It is likely that, if such a correlation exists, the uncertainties on the values of $\Delta_{\text {res }}$ are too large.

In order to determine the precise cause of the smooth patterns, detailed forward seismic modeling is required for each individual star. In addition, each of the found spacing patterns reveals a clear slope. This is as predicted theoretically and points towards frequency shifts of the g-modes due to rotational effects, resulting in a downward or upward slope for prograde and retrograde modes, respectively.

Our results based on the Kepler time series photometry were further confronted with the spectroscopic 


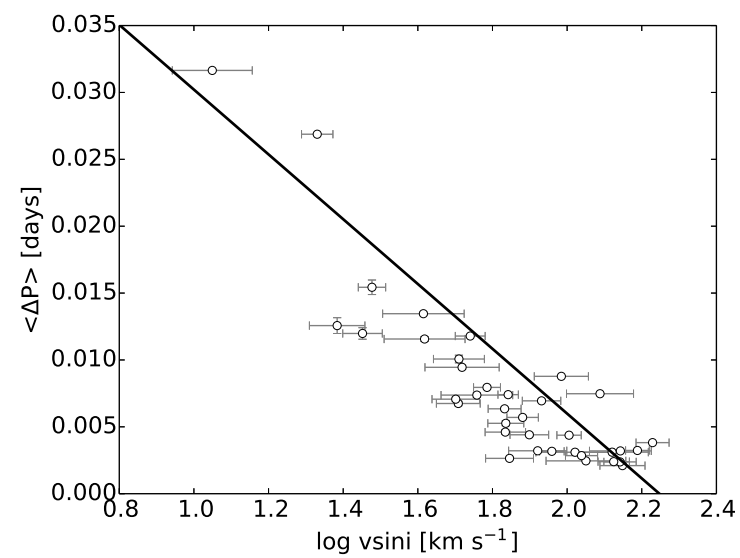

Fig. 8. - The average period spacing values of the different stars with respect to the logarithm of their $v \sin i$ values.
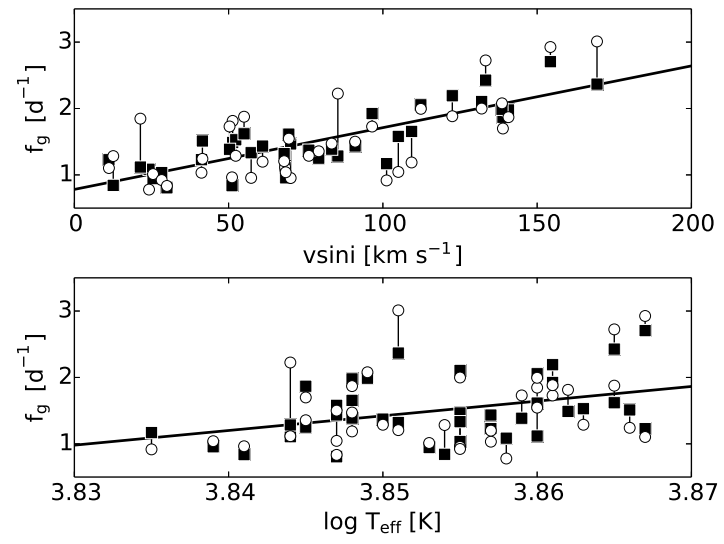

Fig. 9.- Observed values (white dots) for the dominant frequency $f_{g}$, with lines connected to the values predicted by the multivariate models (black squares) described in Table 2 for the rotation velocity $v \sin i$ (top) and the temperature $\log T_{\text {eff }}$ (bottom). The bivariate model fits listed in the left part of Table 2 are shown by the full lines. 

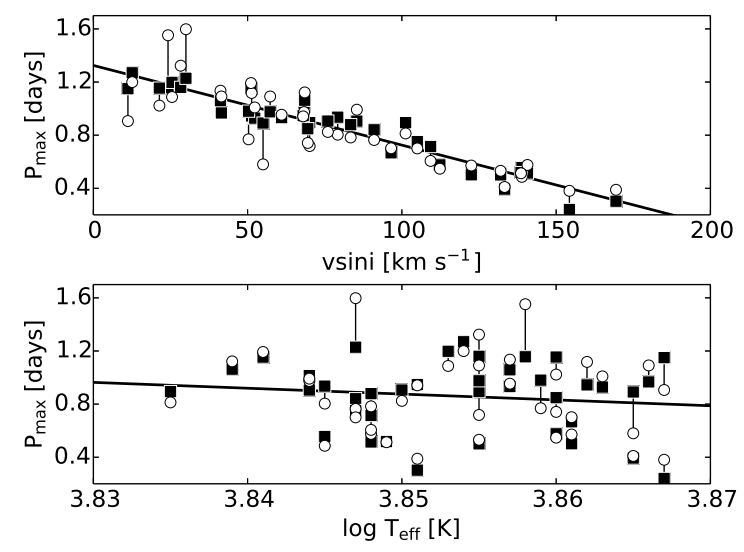

Fig. 10. - Same as Fig. 9, but for the pulsation period $P_{\max }$ instead of the frequency $f_{g}$.
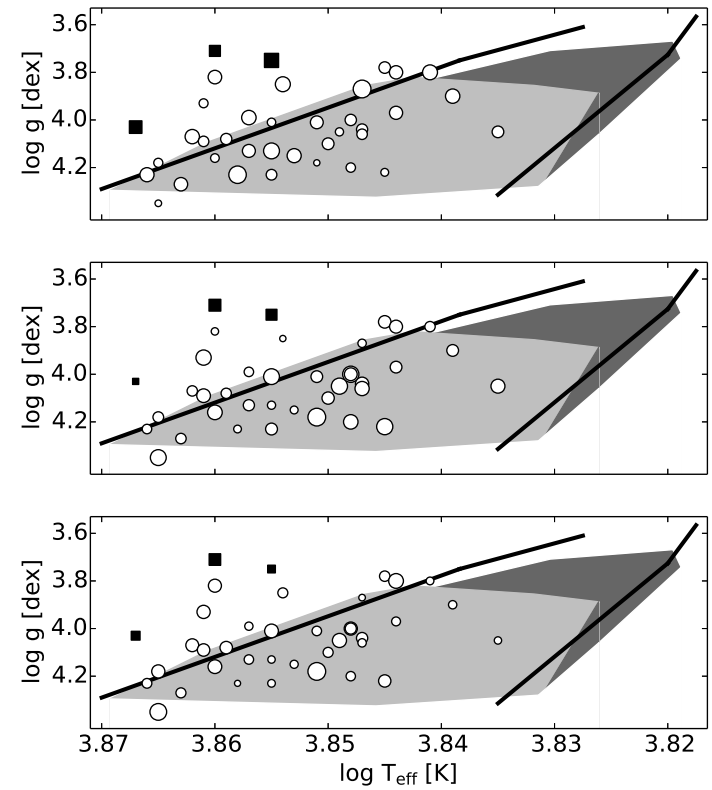

Fig. 11.- $\left(\log T_{\text {eff }}, \log g\right)$-diagrams with the sample stars for which period spacing patterns were found. The white dots indicate the single stars, whereas the black squares are the single-lined binaries. The full lines mark the instability strip taken from Dupret et al. (2005) (with $\alpha_{\mathrm{MLT}}=2.0$ ). The light grey and dark grey areas are the instability strips for the main-sequence $\gamma$ Dor stars presented by Bouabid et al. (2011), with $\alpha_{\mathrm{ov}}=0$ and $\alpha_{\mathrm{ov}}=0.2$ respectively. The marker sizes indicate the longest pulsation period $P_{\max }$ in the spacing patterns (top), the rotation velocity $v \sin i$ (middle) and the g-mode frequency $f_{g}$ with the highest amplitude (bottom). 
parameters of the pulsators. We searched for correlations between the seismic quantities for the stars exhibiting period spacing patterns with a downward slope, since only these constitute a large enough sample to perform multivariate linear regressions. We found a strong correlation between $v \sin i$ and the average period spacing, confirming the influence of rotation on $\gamma$ Dor-type pulsations as predicted by theory. In addition, we found a correlation between the dominant g-mode frequency and both $v \sin i$ and $\log T_{\text {eff }}$. Similarly, $P_{\max }$ correlates with $v \sin i$ and $\log T_{\text {eff }}$.

While our sample contains only eleven hybrid $\gamma$ Dor $/ \delta$ Sct pulsators, these constitute an interesting subsample for various reasons. First, seven of these stars were found to be in a binary. At least four of them contain a hybrid pulsator as three were identified as single-lined binaries, while in the case of KIC 3952623 the observed $\gamma$ Dor and $\delta$ Sct variability originates from the primary component.

Two of the single stars (KIC 8645874 and KIC 9751996) and one of the single-lined binaries (KIC 11754232) were found to be slow rotators. Interestingly, some of the recently studied hybrid $\gamma$ Dor pulsators are also very slow rotators (Kurtz et al. 2014; Bedding et al. 2014; Saio et al. 2015). On the other hand, the hybrid star KIC 4749989 is a fast rotator, similar to KIC 8054146 (e.g. Breger et al. 2012).

We compared the locations of the sample stars in the Kiel diagram with the $\gamma$ Dor instability strips determined by Dupret et al. (2005) and Bouabid et al. (2011) (as shown in Fig.11). Given the large uncertainties on $\log g$, the positions are compatible with the strips, although most of our sample stars clearly cluster around the blue edge. The symbol sizes in these plots indicate the longest pulsation period $P_{\max }$ in the spacing patterns (top), the rotation velocity $v \sin i$ (middle) and the g-mode frequency $f_{g}$ with the largest amplitude (bottom). The quantity $P_{\max }$ is of interest to understand $\gamma$ Dor instability, in the sense that modes with even longer period are more sensitive to radiative damping and/or wave energy leakage.

While we found a correlation between $f_{g}$ as well as $P \max$ and $\log T_{\text {eff }}$ in Section 5 , this is not obvious from the figure because of the strong influence of $v \sin i$ on these two quantities and due to the large errors of $\log g$. We do see from the symbol sizes that a higher rotation velocity typically correlates with a higher frequency $f_{g}$ and a lower period $P_{\max }$, as found from the forward and backward modelling.

Our sample study constitutes a very rich observational data base to persue forward asteroseismic modeling of $\gamma$ Dor stars. All the necessary data to perform such stellar modelling is provided to the community through https://fys. kuleuven.be/ster/Projects/gamma-doradus.

The research leading to these results was based on funding from the Fund for Scientific Research of Flanders (FWO), Belgium under grant agreement G.0B69.13, from the European Communitys Seventh Framework Programme FP7-SPACE-2011-1 project number 312844 (SPACEINN), from the Research Council of KU Leuven under grant GOA/2013/012, and from the National Science Foundation of the USA under grant No. NSF PHY11-25915. KZ acknowledges support by the Austrian Fonds zur Förderung der wissenschaftlichen Forschung (FWF, project V431-NBL). SB is supported by the Foundation for Fundamental Research on Matter (FOM), which is part of the Netherlands Organisation for Scientific Research (NWO). TVR thanks Aaron Greenwood (University of Groningen, the Netherlands) for the very useful discussions on the interaction of rotation and pulsations in $\gamma$ Dor stars. CA, SB, PP and VSS are grateful to the 
Kavli Institute of Theoretical Physics at the University of California, Santa Barbara, for the kind hospitality during the research programme "Galactic Archaeology and Precision Stellar Astrophysics" during which the current study was finalized. Funding for the Kepler mission is provided by NASA's Science Mission Directorate. We thank the whole team for the development and operations of the mission. This research made use of the SIMBAD database, operated at CDS, Strasbourg, France, and the SAO/NASA Astrophysics Data System. This research has also made use of the VizieR catalogue access tool, CDS, Strasbourg, France, and of the VALD database, operated at Uppsala University, the Institute of Astronomy RAS in Moscow, and the University of Vienna.

\section{REFERENCES}

Aerts, C., Christensen-Dalsgaard, J., \& Kurtz, D. W. 2010, Asteroseismology, Astronomy and Astrophsyics Library, Springer Berlin Heidelberg

Aerts, C., Molenberghs, G., Kenward, M. G., \& Neiner, C. 2014, ApJ, 781, 88

Auvergne, M., Bodin, P., Boisnard, L., et al. 2009, A\&A, 506, 411

Balona, L. A., Guzik, J. A., Uytterhoeven, K., et al. 2011, MNRAS, 415, 3531

Baran, A. S., Telting, J. H., Németh, P., Bachulski, S., \& Krzesiński, J. 2015, A\&A, 573, A52

Beck, P. G., Bedding, T. R., Mosser, B., et al. 2011, Science, 332, 205

Beck, P. G., Montalban, J., Kallinger, T., et al. 2012, Nature, 481, 55

Bedding, T. R., Mosser, B., Huber, D., et al. 2011, Nature, 471, 608

Bedding, T. R., Murphy, S. J., Colman, I. L., \& Kurtz, D. W. 2014, arXiv:1411.1883

Bouabid, M.-P., Montalbán, J., Miglio, A., et al. 2011, A\&A, 531, A145

Bouabid, M.-P., Dupret, M.-A., Salmon, S., et al. 2013, MNRAS, 429, 2500

Bradley, P. A., Guzik, J. A., Miles, L. F., et al. 2015, AJ, 149, 68

Breger, M., Fossati, L., Balona, L., et al. 2012, ApJ, 759, 62

Chapellier, E., Mathias, P., Weiss, W. W., Le Contel, D., \& Debosscher, J. 2012, A\&A, 540, A117

Crowe, R. A. \& Mitalas, R. 1982, A\&A, 108, 55

Cuypers, J., Aerts, C., De Cat, P., et al. 2009, A\&A, 499, 967

De Cat, P. \& Aerts, C. 2002, A\&A, 393, 965

Debosscher, J., Aerts, C., Tkachenko, A., et al. 2013, A\&A, 556, A56 
Degroote, P., Aerts, C., Baglin, A., et al. 2010, Nature, 464, 259

Deheuvels, S., Doğan, G., Goupil, M. J., et al. 2014, A\&A, 564, A27

Dupret, M.-A., Grigahcène, A., Garrido, R., Gabriel, M., \& Scuflaire, R. 2005, A\&A, 435, 927

Gray, D. F. 1992, The observation and analysis of stellar photospheres.

Grigahcène, A., Antoci, V., Balona, L., et al. 2010, ApJ, 713, L192

Guzik, J. A., Kaye, A. B., Bradley, P. A., Cox, A. N., \& Neuforge, C. 2000, ApJ, 542, L57

Handler, G. 1999, MNRAS, 309, L19

Handler, G. \& Shobbrook, R. R. 2002, MNRAS, 333, 251

Hansen, C. J., Winget, D. E., \& Kawaler, S. D. 1985, ApJ, 297, 544

Herwig, F. 2000, A\&A, 360, 952

Kaye, A. B., Handler, G., Krisciunas, K., Poretti, E., \& Zerbi, F. M. 1999, PASP, 111, 840

Koch, D. G., Borucki, W. J., Basri, G., et al. 2010, ApJ, 713, L79

Kupka, F., Piskunov, N., Ryabchikova, T. A., Stempels, H. C., \& Weiss, W. W. 1999, A\&AS, 138, 119

Kurtz, D. W., Hambleton, K. M., Shibahashi, H., Murphy, S. J., \& Prša, A. 2015, MNRAS, 446, 1223

Kurtz, D. W., Saio, H., Takata, M., et al. 2014, MNRAS, 444, 102

Lehmann, H., Tkachenko, A., Semaan, T., et al. 2011, A\&A, 526, A124

Meibom, S., Barnes, S. A., Platais, I., et al. 2015, Nature, 517, 589

Miglio, A., Montalbán, J., Noels, A., \& Eggenberger, P. 2008, MNRAS, 386, 1487

Mitalas, R. 1972, ApJ, 177, 693

Morel, T., Butler, K., Aerts, C., Neiner, C., \& Briquet, M. 2006, A\&A, 457, 651

Mosser, B., Goupil, M. J., Belkacem, K., et al. 2012, A\&A, 548, A10

Murphy, S. J., Bedding, T. R., Shibahashi, H., Kurtz, D. W., \& Kjeldsen, H. 2014, MNRAS, 441, 2515

Murphy, S. J., Shibahashi, H., \& Kurtz, D. W. 2013, MNRAS, 430, 2986

Pápics, P. I., Briquet, M., Baglin, A., et al. 2012, A\&A, 542, A55

Pápics, P. I., Moravveji, E., Aerts, C., et al. 2014, A\&A, 570, A8

Paxton, B., Bildsten, L., Dotter, A., et al. 2011, ApJS, 192, 3 
Paxton, B., Cantiello, M., Arras, P., et al. 2013, ApJS, 208, 4

Raskin, G., van Winckel, H., Hensberge, H., et al. 2011, A\&A, 526, A69

Reed, M. D., Foster, H., Telting, J. H., et al. 2014, MNRAS, 440, 3809

Saio, H., Kurtz, D. W., Takata, M., et al. 2015, MNRAS, 447, 3264

Savonije, G. J. 2013, A\&A, 559, A25

Schwarzenberg-Czerny, A. 2003, in Astronomical Society of the Pacific Conference Series, Vol. 292, Interplay of Periodic, Cyclic and Stochastic Variability in Selected Areas of the H-R Diagram, ed. C. Sterken, 383

Shibahashi, H. \& Kurtz, D. W. 2012, MNRAS, 422, 738

Shulyak, D., Tsymbal, V., Ryabchikova, T., Stütz, C., \& Weiss, W. W. 2004, A\&A, 428, 993

Silva Aguirre, V., Ballot, J., Serenelli, A. M., \& Weiss, A. 2011, A\&A, 529, A63

Tassoul, M. 1980, ApJS, 43, 469

Tkachenko, A., Lehmann, H., Smalley, B., Debosscher, J., \& Aerts, C. 2012, MNRAS, 422, 2960

Tkachenko, A., Aerts, C., Yakushechkin, A., et al. 2013a, A\&A, 556, A52

Tkachenko, A., Lehmann, H., Smalley, B., \& Uytterhoeven, K. 2013b, MNRAS, 431, 3685

Tkachenko, A., Van Reeth, T., Tsymbal, V., et al. 2013c, A\&A, 560, A37

Townsend, R. H. D. 2000, MNRAS, 319, 289

Townsend, R. H. D. 2003, MNRAS, 343, 125

Townsend, R. H. D. \& Teitler, S. A. 2013, MNRAS, 435, 3406

Triana, S. A., Moravveji, E., Pápics, P. I., et al., 2015, ApJ, submitted

Tsymbal, V. 1996, in Astronomical Society of the Pacific Conference Series, Vol. 108, M.A.S.S., Model Atmospheres and Spectrum Synthesis, ed. S. J. Adelman, F. Kupka, \& W. W. Weiss, 198

Uytterhoeven, K., Moya, A., Grigahcène, A., et al. 2011, A\&A, 534, A125

Van Reeth, T., Tkachenko, A., Aerts, C., et al. 2015, A\&A, 574, A17

Walker, G., Matthews, J., Kuschnig, R., et al. 2003, Publications of the Astronomical Society of the Pacific, 115,1023

Zwintz, K., Fossati, L., Ryabchikova, T., et al. 2014, Science, 345, 550 
Table A1. Observation log of the stars in our sample. $N_{Q}$ and $N_{S}$ indicate the total number of available Kepler mission quarters and HERMES spectra respectively.

\begin{tabular}{|c|c|c|c|c|c|c|c|}
\hline KIC & RA (J2000) & DEC (J2000) & $m_{V}$ & $N_{Q}$ & Available quarters & $N_{S}$ & Spectroscopic observation times \\
\hline 2710594 & 192333.317 & +375900.55 & 11.79 & 18 & $0-17$ & 4 & May-August 2011 \\
\hline 3222854 & 191138.539 & +382043.83 & 12.5 & 18 & $0-17$ & 7 & August 2013 \\
\hline 3448365 & 193035.779 & +383516.67 & 9.92 & 18 & & 4 & May-August 2011 \\
\hline 3744571 & 192305.597 & +384851.91 & 12.03 & 18 & $0-17$ & 4 & June-July 2013 \\
\hline 3952623 & 192408.225 & +390040.25 & 12.09 & 18 & $0-17$ & 14 & May-July 2013, July-August 2014 \\
\hline 4547348 & 190818.888 & +394136.53 & 11.78 & 18 & $0-17$ & 4 & May-August 2011 \\
\hline 4749989 & 193105.251 & +395224.78 & 9.64 & 150 & $0-5,7-9,11-13,15-17$ & 2 & May-June 2011 \\
\hline 4757184 & 193836.922 & +395026.10 & 11.7 & 8 & $0-1,11-13,15-17$ & 5 & May-August 2011 \\
\hline 4846809 & 193919.027 & +395510.70 & 12.36 & 150 & $0-5,7-9,11-13,15-17$ & 4 & June-July 2 \\
\hline 5000454 & 191 & +401044.76 & 13.56 & 17 & $1-17$ & 3 & Jul \\
\hline 5114382 & 194243.543 & +401500.66 & 11.55 & 8 & $0-1,11-13,15-17$ & 4 & July-Au \\
\hline 5254203 & 185608.364 & +402712.87 & 12.92 & 14 & $0-4,6-8,10-12,14-16$ & 4 & August 2013, June-July 2014 \\
\hline 5350598 & 191154.360 & +403401.78 & 12.1 & 18 & $0-17$ & 4 & June-July 2013 \\
\hline 5522154 & 191223.386 & +404214.00 & 10.44 & 10 & $0-1,10-17$ & 3 & May-June 2011 \\
\hline 5708550 & 193103.494 & +405613.89 & 11.93 & 18 & $0-17$ & 4 & July-August 2011 \\
\hline 5772452 & 190004.541 & +410517.20 & 12.46 & 140 & $0-4,6-8,10-12,14-16$ & 2 & August-September 2013 \\
\hline 5788623 & 192411.066 & +410041.47 & 12.07 & 18 & $0-17$ & 4 & June-J \\
\hline 6185513 & 185729.040 & +413058.10 & 11.89 & 8 & $0-1,10-12,14-16$ & 4 & May 2011 \\
\hline 6342398 & 185134.322 & +414635.05 & 12.37 & 140 & $0-4,6-8,10-12,14-16$ & 4 & May-June 2013 \\
\hline 6367159 & 193121.144 & +414455.50 & 12.6 & 18 & $0-17$ & 8 & August-September 2013, June-August 2014 \\
\hline 6425437 & 190003.295 & +415016.36 & 11.48 & 140 & $0-4,6-8,10-12,14-16$ & 3 & May 2011 \\
\hline 6467639 & 195337.829 & +414905.02 & 12.02 & 18 & $0-17$ & 12 & June-July 2013, July-August 2014 \\
\hline 6468146 & 195404.241 & +414831.01 & 9.96 & 18 & & 4 & July 2011, June-August 2014 \\
\hline 6468987 & 195449.006 & +414813.54 & 12.67 & 18 & $0-17$ & 4 & August 2013, June-August 2014 \\
\hline 6678174 & 191005.712 & +420954.58 & 11.74 & 10 & $-1,10-17$ & 4 & May-August 2011 \\
\hline 6778063 & 192806.622 & +421529.60 & 12.18 & 18 & $0-17$ & 9 & June-July 2013, July-August 2014 \\
\hline 6935014 & 190839.984 & +422711.77 & 10.91 & 18 & $0-17$ & 5 & May-June 2011 \\
\hline 6953103 & 193251.240 & +422846.53 & 12.56 & 18 & $0-17$ & 4 & August-September 2013, June-August 2014 \\
\hline 7023122 & 191342.062 & +423113.02 & 10.84 & 18 & $0-17$ & 4 & May-July 2011 \\
\hline
\end{tabular}


Table A1-Continued

\begin{tabular}{|c|c|c|c|c|c|c|c|}
\hline KIC & RA (J2000) & DEC (J2000) & $m_{V}$ & $N_{Q}$ & Available quarters & $N_{S}$ & Spectroscopic observation times \\
\hline 7365537 & 193035.237 & +425810.69 & 9.19 & 18 & $0-17$ & 3 & May 2011 \\
\hline 7380501 & 194641.587 & +425935.34 & 11.98 & 18 & $0-17$ & 4 & July-August 2011 \\
\hline 7434470 & 191357.941 & +430457.98 & 12.02 & 18 & $0-17$ & 4 & June-July 2013 \\
\hline 7516703 & 191248.742 & +431154.34 & 12.51 & 13 & $1-3,6-7,10-17$ & 2 & August-September 2013 \\
\hline 7583663 & 184657.458 & +431615.31 & 12.69 & 18 & $0-17$ & 4 & August 2013, June-July 2014 \\
\hline 7691618 & 193902.666 & +431901.20 & 12.36 & 18 & $0-17$ & 4 & June-July 2013 \\
\hline 7746984 & 191845.175 & +432401.54 & 12.47 & 100 & $0-1,3,5,7,9,11,13,15,17$ & 2 & August 2013 \\
\hline 7867348 & 184040.774 & +433617.09 & 10.84 & 18 & $0-17$ & 12 & May 2011, June-August 2014 \\
\hline 7939065 & 184911.976 & +434753.52 & 12.14 & 18 & $0-17$ & 4 & May-June 2013 \\
\hline 8364249 & 192516.824 & +442346.75 & 11.95 & 18 & $0-17$ & 4 & July-August 2011 \\
\hline 8375138 & 194047.623 & +442011.83 & 11.02 & 18 & $0-17$ & 4 & May-August 2011 \\
\hline 8378079 & 194430.607 & +442258.04 & 11.84 & 18 & $0-17$ & 3 & August 2011 \\
\hline 8611423 & 190312.216 & +444435.05 & 11.59 & 10 & $0-1,10-17$ & 4 & May-June 2011 \\
\hline 8645874 & 195351.715 & +444309.37 & 9.92 & 10 & $0-1,10-17$ & 2 & July 2011 \\
\hline 8693972 & 193444.590 & +445224.03 & 12.81 & 18 & $0-17$ & 7 & August-September 2013, July-August 2014 \\
\hline 8739181 & 190006.043 & +445643.47 & 12.08 & 18 & $0-17$ & 5 & June-July 2013 \\
\hline 8836473 & 195112.305 & +450134.10 & 12.78 & 18 & $0-17$ & 4 & August 2013, June-August 2014 \\
\hline 9210943 & 191404.512 & +453908.99 & 11.81 & 18 & $0-17$ & 5 & May-August 2011, June-August 2014 \\
\hline 9419694 & 194957.994 & +45 5707.09 & 12.74 & 15 & $0-6,8-10,12-14,16-17$ & 4 & August 2013, June-August 2014 \\
\hline 9480469 & 194934.555 & +460420.90 & 12.78 & 15 & $0-6,8-10,12-14,16-17$ & 5 & August-September 2013, June-August 2014 \\
\hline 9595743 & 193919.226 & +461457.49 & 12.09 & 11 & $0-1,5,10-17$ & 4 & June-July 2013 \\
\hline 9751996 & 184559.486 & +463043.23 & 10.96 & 10 & $0-1,10-17$ & 4 & May 2011 \\
\hline 10080943 & 193627.305 & +470502.29 & 11.81 & 18 & $0-17$ & 27 & August 2011, May-July 2013, July-October 2014 \\
\hline 10224094 & 195006.643 & +471706.58 & 11.9 & 15 & $0-6,8-10,12-14,16-17$ & 4 & July-August 2011 \\
\hline 10256787 & 185347.189 & +472335.01 & 12.25 & 18 & $0-17$ & 14 & May-June 2013, June-August 2014 \\
\hline 10467146 & 192206.050 & +474049.33 & 12.66 & 18 & $0-17$ & 4 & August-September 2013, June-August 2014 \\
\hline 11080103 & 191850.141 & +483713.90 & 12.94 & 18 & $0-17$ & 4 & August-September 2013, June-August 2014 \\
\hline 11099031 & 195237.514 & +483844.01 & 10.02 & 15 & $0-6,8-10,12-14,16-17$ & 4 & July 2011 \\
\hline 11196370 & 193844.486 & +485055.64 & 12.71 & 18 & $0-17$ & 2 & August-September 2013 \\
\hline
\end{tabular}


Table A1-Continued

\begin{tabular}{|c|c|c|c|c|c|c|c|}
\hline $\mathrm{KIC}$ & RA (J2000) & DEC (J2000) & $m_{V}$ & $N_{Q}$ & Available quarters & $N_{S}$ & Spectroscopic observation times \\
\hline 11294808 & 192245.396 & +490447.53 & 11.73 & 18 & $0-17$ & 4 & May-August 2011 \\
\hline 11456474 & 193010.495 & +492028.06 & 12.49 & 18 & $0-17$ & 4 & August-September 2013, June-August 2014 \\
\hline 11668783 & 194127.422 & +494741.24 & 12.58 & 18 & $0-17$ & 4 & August-September 2013, June-August 2014 \\
\hline 11721304 & 194327.847 & +495125.41 & 11.71 & 18 & $0-17$ & 4 & July-August 2011 \\
\hline 11754232 & 190557.926 & +495633.53 & 12.24 & 8 & $0-1,10-11,13-15,17$ & 13 & June-July 2013, June-August 2014 \\
\hline 11826272 & 194950.494 & +500057.36 & 10.21 & 18 & $0-17$ & 3 & May-August 2011 \\
\hline 11907454 & 191129.407 & +501429.72 & 11.38 & 18 & $0-17$ & 4 & May-August 2011 \\
\hline 11917550 & 193422.565 & +501546.29 & 11.12 & 18 & $0-17$ & 4 & May-August 2011 \\
\hline 11920505 & 193957.108 & +501332.09 & 9.88 & 18 & $0-17$ & 2 & May 2011 \\
\hline 12066947 & 193703.209 & +503020.41 & 10.23 & 10 & $0-1,10-17$ & 3 & May-July 2011 \\
\hline 12458189 & 192049.123 & +512009.25 & 11.46 & 18 & $0-17$ & 4 & May-August 2011 \\
\hline 12643786 & 191349.205 & +514648.32 & 11.52 & 18 & $0-17$ & 4 & May-August 2011 \\
\hline
\end{tabular}


Table A2. The global observational characteristics of the stars in our sample. We have listed information on the variability type, where the label "ROT" indicates rotational modulation, and * indicates that frequencies are found outside of the traditional range for $\gamma$ Dor stars, but likely correspond to combination frequencies. In addition, stars are listed as single (S), single-lined binaries (SB1), double-lined binaries (SB2), binaries with no detected radial velocity variations (SB) and triple systems (SB3). We also report the detections of period spacing patterns and comment on their suspected nature.

\begin{tabular}{|c|c|c|c|c|}
\hline $\mathrm{KIC}$ & Class & binarity & Period spacings & Comments \\
\hline 2710594 & $\gamma$ Dor & S & yes & Prograde and retrograde \\
\hline 3222854 & $\gamma$ Dor & SB2 & no & - \\
\hline 3448365 & $\gamma$ Dor & $S$ & yes & Prograde and retrograde \\
\hline 3744571 & $\gamma$ Dor & S & no & - \\
\hline 3952623 & $\gamma$ Dor $/ \delta$ Sct hybrid & SB2 & no & - \\
\hline 4547348 & $\gamma$ Dor & S & no & - \\
\hline 4749989 & $\gamma$ Dor $/ \delta$ Sct hybrid & S & no & - \\
\hline 4757184 & $\gamma$ Dor & S & yes & Retrograde (quadrupole) \\
\hline 4846809 & $\gamma$ Dor & S & yes & Prograde (dipole and quadrupole) \\
\hline 5000454 & $\gamma$ Dor & $S$ & no & - \\
\hline 5114382 & $\gamma$ Dor & S & yes & Prograde and retrograde \\
\hline 5254203 & $\gamma$ Dor & $S$ & no & - \\
\hline 5350598 & $\gamma$ Dor & S & yes & Retrograde (quadrupole) \\
\hline 5522154 & $\gamma$ Dor* & S & yes & Prograde \\
\hline 5708550 & $\gamma$ Dor & S & yes & Prograde \\
\hline 5772452 & K-type (ROT) & - & - & - \\
\hline 5788623 & $\gamma$ Dor & $S$ & yes & Prograde \\
\hline 6185513 & $\gamma$ Dor* & S & yes & Retrograde (quadrupole) \\
\hline 6342398 & $\gamma$ Dor & S & no & - \\
\hline 6367159 & $\gamma$ Dor $/ \delta$ Sct hybrid & SB2 & no & - \\
\hline 6425437 & $\gamma$ Dor & $\mathrm{S}$ & yes & Retrograde (quadrupole) \\
\hline 6467639 & $\gamma$ Dor $/ \delta$ Sct hybrid & SB3 & no & - \\
\hline 6468146 & $\gamma$ Dor $/ \delta$ Sct hybrid & SB1 & yes & Prograde \\
\hline 6468987 & $\gamma$ Dor & $S$ & yes & Prograde and retrograde \\
\hline 6678174 & $\gamma$ Dor & $S$ & yes & Prograde \\
\hline 6778063 & $\gamma$ Dor $/ \delta$ Sct hybrid & SB3 & yes & Prograde \\
\hline 6935014 & $\gamma$ Dor & $S$ & yes & Prograde \\
\hline 6953103 & $\gamma$ Dor & S & yes & Prograde \\
\hline 7023122 & $\gamma$ Dor & S & yes & Prograde \\
\hline 7365537 & $\gamma$ Dor* & $S$ & yes & Prograde \\
\hline 7380501 & $\gamma$ Dor & $S$ & yes & Prograde \\
\hline 7434470 & $\gamma$ Dor* & S & yes & Prograde and strong rotation frequency \\
\hline 7516703 & K-type (ROT) & - & - & - \\
\hline
\end{tabular}


Table A2-Continued

\begin{tabular}{|c|c|c|c|c|}
\hline KIC & Class & binarity & Period spacings & Comments \\
\hline 7583663 & $\gamma$ Dor & S & yes & Prograde and retrograde \\
\hline 7691618 & $\gamma$ Dor & $\mathrm{S}$ & no & - \\
\hline 7746984 & $\gamma$ Dor & SB & yes & Prograde \\
\hline 7867348 & $\gamma$ Dor $/ \delta$ Sct hybrid & SB1 & yes & Retrograde (quadrupole) \\
\hline 7939065 & $\gamma$ Dor* & $S$ & yes & Prograde \\
\hline 8364249 & $\gamma$ Dor & S & yes & Prograde \\
\hline 8375138 & $\gamma$ Dor* & S & yes & Prograde and retrograde \\
\hline 8378079 & $\gamma$ Dor & S & no & - \\
\hline 8611423 & $\gamma$ Dor & S & no & - \\
\hline 8645874 & $\gamma$ Dor $/ \delta$ Sct hybrid & S & yes & Prograde \\
\hline 8693972 & $\gamma$ Dor & SB2 & no & - \\
\hline 8739181 & $\gamma$ Dor & S & no & - \\
\hline 8836473 & $\gamma$ Dor $/ \delta$ Sct hybrid & S & yes & Prograde \\
\hline 9210943 & $\gamma$ Dor & SB & yes & Prograde and retrograde \\
\hline 9419694 & $\gamma$ Dor & $\mathrm{S}$ & yes & Prograde \\
\hline 9480469 & $\gamma$ Dor & S & yes & Prograde and retrograde \\
\hline 9595743 & $\gamma$ Dor & S & yes & Prograde \\
\hline 9751996 & $\gamma$ Dor $/ \delta$ Sct hybrid & S & yes & Prograde, retrograde and zonal \\
\hline 10080943 & $\gamma$ Dor $/ \delta$ Sct hybrid & SB2 & - & - \\
\hline 10224094 & $\gamma$ Dor & S & yes & Prograde \\
\hline 10256787 & $\gamma$ Dor & SB & yes & Prograde \\
\hline 10467146 & $\gamma$ Dor & SB1 & yes & Prograde \\
\hline 11080103 & $\gamma$ Dor & $\mathrm{S}$ & yes & Prograde \\
\hline 11099031 & $\gamma$ Dor* & S & yes & Prograde \\
\hline 11196370 & $\gamma$ Dor* & S & no & - \\
\hline 11294808 & $\gamma$ Dor* & S & yes & Prograde (dipole and quadrupole) \\
\hline 11456474 & $\gamma$ Dor & S & yes & Prograde \\
\hline 11668783 & $\gamma$ Dor & S & yes & Retrograde \\
\hline 11721304 & $\gamma$ Dor & S & yes & Prograde \\
\hline 11754232 & $\gamma$ Dor $/ \delta$ Sct hybrid & SB1 & yes & Prograde \\
\hline 11826272 & $\gamma$ Dor & S & yes & Prograde \\
\hline 11907454 & $\gamma$ Dor & S & yes & Prograde and retrograde \\
\hline 11917550 & $\gamma$ Dor & S & yes & Prograde \\
\hline
\end{tabular}


Table A2-Continued

\begin{tabular}{ccccl}
\hline \hline KIC & Class & binarity & Period spacings & Comments \\
\hline 11920505 & $\gamma$ Dor & $\mathrm{S}$ & yes & Prograde \\
12066947 & $\gamma$ Dor* & $\mathrm{S}$ & yes & Prograde and retrograde \\
12458189 & $\gamma$ Dor* & $\mathrm{S}$ & yes & Prograde \\
12643786 & $\gamma$ Dor & $\mathrm{S}$ & no & - \\
\hline
\end{tabular}


Table A3. Spectroscopic parameter values computed for the merged spectra of the single stars and single-lined binaries in our sample. The provided errors are the $1-\sigma$ values obtained from the $\chi^{2}$-statistics.

\begin{tabular}{|c|c|c|c|c|c|c|c|c|c|c|c|c|}
\hline KIC & $\log T_{\text {eff }}[\mathrm{K}]$ & $\sigma_{\log T_{\text {eff }}}$ & $T_{\text {eff }}[\mathrm{K}]$ & $\sigma_{T_{\text {eff }}}$ & $\log g$ & $\sigma_{\log g}$ & $\zeta\left[\mathrm{km} \mathrm{s}^{-1}\right]$ & $\sigma_{\zeta}$ & $v \sin i\left[\mathrm{~km} \mathrm{~s}^{-1}\right]$ & $\sigma_{v \sin i}$ & {$[M / H]$} & $\sigma_{[M / H]}$ \\
\hline 2710594 & 3.845 & 0.006 & 7000 & 100 & 3.78 & 0.33 & 2.93 & 0.42 & 79.2 & 4.1 & 0.11 & 0.09 \\
\hline 3448365 & 3.847 & 0.004 & 7030 & 60 & 4.04 & 0.22 & 2.52 & 0.26 & 91.0 & 3.0 & 0.05 & 0.07 \\
\hline 3744571 & 3.850 & 0.008 & 7075 & 130 & 3.94 & 0.40 & 2.47 & 0.58 & 52.5 & 4.0 & 0.11 & 0.11 \\
\hline 4547348 & 3.850 & 0.006 & 7080 & 105 & 4.02 & 0.34 & 2.54 & 0.44 & 68.5 & 4.0 & 0.10 & 0.09 \\
\hline 4749989 & 3.865 & 0.003 & 7320 & 55 & 4.24 & 0.21 & 3.33 & 0.22 & 204.0 & 8.0 & 0.13 & 0.06 \\
\hline 4757184 & 3.862 & 0.008 & 7285 & 135 & 4.16 & 0.43 & 1.90 & 0.63 & 36.0 & 3.0 & 0.02 & 0.13 \\
\hline 4846809 & 3.862 & 0.008 & 7280 & 135 & 4.07 & 0.32 & 3.00 & 0.59 & 51.3 & 3.5 & 0.18 & 0.11 \\
\hline 5000454 & 3.840 & 0.006 & 6920 & 100 & 3.46 & 0.38 & 3.91 & 0.50 & 101.0 & 4.4 & 0.07 & 0.10 \\
\hline 5114382 & 3.855 & 0.007 & 7165 & 120 & 4.23 & 0.37 & 2.45 & 0.45 & 70.1 & 4.5 & 0.19 & 0.10 \\
\hline 5254203 & 3.866 & 0.014 & 7350 & 235 & 4.09 & 0.72 & 3.11 & 1.10 & 42.7 & 6.0 & 0.21 & 0.21 \\
\hline 5350598 & 3.864 & 0.007 & 7315 & 110 & 4.11 & 0.33 & 2.64 & 0.42 & 27.8 & 1.7 & 0.10 & 0.09 \\
\hline 5522154 & 3.851 & 0.004 & 7090 & 70 & 4.18 & 0.26 & 3.15 & 0.36 & 169.4 & 7.5 & 0.08 & 0.08 \\
\hline 5708550 & 3.844 & 0.006 & 6990 & 100 & 3.97 & 0.32 & 2.44 & 0.42 & 68.3 & 3.7 & 0.15 & 0.09 \\
\hline 5788623 & 3.858 & 0.007 & 7210 & 115 & 4.23 & 0.32 & 2.20 & 0.40 & 24.2 & 1.8 & 0.09 & 0.09 \\
\hline 6185513 & 3.867 & 0.007 & 7365 & 120 & 4.10 & 0.40 & 3.91 & 0.72 & 82.9 & 5.5 & 0.16 & 0.11 \\
\hline 6342398 & 3.855 & 0.010 & 7165 & 165 & 3.95 & 0.49 & 2.61 & 0.60 & 31.6 & 2.9 & 0.17 & 0.13 \\
\hline 6425437 & 3.848 & 0.007 & 7045 & 112 & 3.88 & 0.35 & 2.64 & 0.43 & 49.7 & 2.5 & 0.21 & 0.09 \\
\hline 6468146 & 3.860 & 0.004 & 7240 & 65 & 3.71 & 0.22 & 3.56 & 0.33 & 69.5 & 1.9 & -0.05 & 0.06 \\
\hline 6468987 & 3.855 & 0.013 & 7155 & 210 & 4.01 & 0.72 & 4.08 & 1.10 & 132.0 & 12.8 & 0.38 & 0.20 \\
\hline 6678174 & 3.852 & 0.006 & 7105 & 90 & 3.77 & 0.33 & 2.59 & 0.35 & 45.5 & 2.3 & 0.02 & 0.08 \\
\hline 6935014 & 3.851 & 0.004 & 7090 & 66 & 4.01 & 0.22 & 2.66 & 0.32 & 68.0 & 3.0 & 0.14 & 0.07 \\
\hline 6953103 & 3.863 & 0.010 & 7290 & 175 & 4.27 & 0.50 & 2.50 & 0.81 & 52.3 & 5.2 & 0.25 & 0.15 \\
\hline 7023122 & 3.865 & 0.005 & 7335 & 82 & 4.18 & 0.30 & 2.81 & 0.43 & 55.0 & 2.2 & 0.04 & 0.08 \\
\hline 7365537 & 3.867 & 0.004 & 7360 & 65 & 4.48 & 0.23 & 3.23 & 0.26 & 154.3 & 4.9 & 0.11 & 0.05 \\
\hline 7380501 & 3.841 & 0.007 & 6930 & 110 & 3.80 & 0.35 & 2.33 & 0.47 & 51.1 & 3.0 & 0.14 & 0.10 \\
\hline
\end{tabular}


Table A3-Continued

\begin{tabular}{|c|c|c|c|c|c|c|c|c|c|c|c|c|}
\hline KIC & $\log T_{\text {eff }}[\mathrm{K}]$ & $\sigma_{\log T_{\text {eff }}}$ & $T_{\mathrm{eff}}[\mathrm{K}]$ & $\sigma_{T_{\mathrm{eff}}}$ & $\log g$ & $\sigma_{\log g}$ & $\zeta\left[\mathrm{km} \mathrm{s}^{-1}\right]$ & $\sigma_{\zeta}$ & $v \sin i\left[\mathrm{~km} \mathrm{~s}^{-1}\right]$ & $\sigma_{v \sin i}$ & {$[M / H]$} & $\sigma_{[M / H]}$ \\
\hline 7434470 & 3.845 & 0.009 & 7005 & 150 & 4.22 & 0.50 & 3.33 & 0.90 & 138.9 & 11.5 & 0.25 & 0.14 \\
\hline 7583663 & 3.847 & 0.014 & 7030 & 230 & 4.06 & 0.66 & 3.38 & 1.20 & 105.0 & 11.0 & 0.42 & 0.20 \\
\hline 7691618 & 3.852 & 0.009 & 7110 & 150 & 4.10 & 0.45 & 2.33 & 0.62 & 43.3 & 3.5 & 0.15 & 0.13 \\
\hline 7867348 & 3.847 & 0.005 & 7030 & 75 & 3.56 & 0.22 & 2.94 & 0.20 & 17.2 & 1.0 & -0.06 & 0.06 \\
\hline 7939065 & 3.861 & 0.009 & 7265 & 145 & 4.09 & 0.48 & 3.38 & 0.78 & 96.5 & 7.0 & 0.24 & 0.13 \\
\hline 8364249 & 3.848 & 0.007 & 7045 & 115 & 4.00 & 0.46 & 3.36 & 0.55 & 140.7 & 8.5 & 0.17 & 0.11 \\
\hline 8375138 & 3.849 & 0.005 & 7070 & 80 & 4.05 & 0.34 & 3.14 & 0.40 & 138.6 & 6.0 & 0.08 & 0.08 \\
\hline 8378079 & 3.845 & 0.011 & 7000 & 170 & 3.31 & 0.55 & 1.75 & 0.47 & 11.7 & 1.2 & -0.14 & 0.14 \\
\hline 8611423 & 3.853 & 0.006 & 7135 & 105 & 4.21 & 0.30 & 1.86 & 0.34 & 20.7 & 1.5 & 0.10 & 0.08 \\
\hline 8645874 & 3.860 & 0.005 & 7240 & 90 & 3.82 & 0.27 & 3.38 & 0.31 & 21.4 & 0.9 & -0.05 & 0.06 \\
\hline 8739181 & 3.848 & 0.007 & 7055 & 120 & 3.59 & 0.40 & 2.24 & 0.44 & 27.4 & 1.8 & -0.02 & 0.10 \\
\hline 8836473 & 3.861 & 0.011 & 7260 & 190 & 3.93 & 0.60 & 4.71 & 1.00 & 122.5 & 11.0 & 0.37 & 0.20 \\
\hline 9419694 & 3.857 & 0.011 & 7200 & 185 & 3.99 & 0.58 & 2.50 & 0.77 & 41.2 & 4.5 & 0.17 & 0.16 \\
\hline 9480469 & 3.860 & 0.014 & 7250 & 240 & 4.16 & 0.70 & 3.74 & 1.25 & 112.3 & 12.0 & 0.59 & 0.25 \\
\hline 9595743 & 3.859 & 0.008 & 7230 & 125 & 4.08 & 0.40 & 2.44 & 0.59 & 50.3 & 3.2 & 0.14 & 0.10 \\
\hline 9751996 & 3.854 & 0.006 & 7145 & 100 & 3.85 & 0.24 & 3.07 & 0.27 & 12.6 & 0.9 & 0.28 & 0.07 \\
\hline 10224094 & 3.853 & 0.006 & 7130 & 100 & 4.15 & 0.30 & 2.25 & 0.39 & 25.5 & 1.5 & 0.07 & 0.08 \\
\hline 10467146 & 3.855 & 0.011 & 7155 & 175 & 3.75 & 0.60 & 2.85 & 0.80 & 57.3 & 5.5 & 0.12 & 0.15 \\
\hline 11080103 & 3.866 & 0.012 & 7345 & 210 & 4.23 & 0.65 & 2.80 & 0.95 & 41.5 & 4.5 & 0.18 & 0.18 \\
\hline 11099031 & 3.835 & 0.004 & 6835 & 70 & 4.05 & 0.20 & 2.60 & 0.20 & 101.2 & 3.3 & 0.20 & 0.05 \\
\hline 11196370 & 3.861 & 0.013 & 7260 & 215 & 4.05 & 0.55 & 2.75 & 0.68 & 16.0 & 2.0 & 0.26 & 0.16 \\
\hline 11294808 & 3.844 & 0.006 & 6975 & 95 & 3.80 & 0.35 & 2.95 & 0.44 & 85.4 & 4.4 & 0.12 & 0.09 \\
\hline 11456474 & 3.848 & 0.010 & 7040 & 160 & 4.00 & 0.50 & 3.21 & 0.79 & 83.4 & 6.5 & 0.20 & 0.14 \\
\hline 11668783 & 3.845 & 0.009 & 7000 & 150 & 4.00 & 0.43 & 2.31 & 0.52 & 33.5 & 2.7 & 0.19 & 0.12 \\
\hline 11721304 & 3.855 & 0.005 & 7160 & 82 & 4.13 & 0.28 & 2.15 & 0.33 & 28.3 & 1.5 & 0.08 & 0.08 \\
\hline
\end{tabular}


Table A3-Continued

\begin{tabular}{cccccccccccccc}
\hline \hline $\mathrm{KIC}$ & $\log T_{\text {eff }}[\mathrm{K}]$ & $\sigma_{\log T_{\text {eff }}}$ & $T_{\text {eff }}[\mathrm{K}]$ & $\sigma_{T_{\text {eff }}}$ & $\log g$ & $\sigma_{\log g}$ & $\zeta\left[\mathrm{km} \mathrm{s}^{-1}\right]$ & $\sigma_{\zeta}$ & $v \sin i\left[\mathrm{~km} \mathrm{~s}^{-1}\right]$ & $\sigma_{v \sin i}$ & {$[M / H]$} & $\sigma_{[M / H]}$ \\
\hline 11754232 & 3.867 & 0.007 & 7360 & 120 & 4.03 & 0.33 & 2.40 & 0.38 & 11.2 & 1.2 & -0.05 & 0.09 \\
11826272 & 3.847 & 0.004 & 7030 & 70 & 3.87 & 0.21 & 2.37 & 0.21 & 30.0 & 1.1 & 0.09 & 0.06 \\
11907454 & 3.848 & 0.006 & 7040 & 90 & 4.20 & 0.32 & 2.45 & 0.27 & 109.3 & 4.7 & 0.16 & 0.08 \\
11917550 & 3.850 & 0.005 & 7080 & 75 & 4.10 & 0.24 & 2.61 & 0.32 & 76.0 & 3.2 & 0.14 & 0.07 \\
11920505 & 3.857 & 0.004 & 7200 & 60 & 4.13 & 0.19 & 2.55 & 0.19 & 61.0 & 2.2 & 0.10 & 0.05 \\
12066947 & 3.865 & 0.004 & 7330 & 70 & 4.35 & 0.30 & 3.40 & 0.33 & 133.3 & 5.6 & 0.09 & 0.07 \\
12458189 & 3.839 & 0.006 & 6895 & 90 & 3.90 & 0.31 & 2.62 & 0.39 & 68.5 & 3.3 & 0.16 & 0.09 \\
12643786 & 3.857 & 0.006 & 7200 & 95 & 4.00 & 0.31 & 2.63 & 0.45 & 76.7 & 4.5 & 0.11 & 0.09 \\
\hline
\end{tabular}


Table A4. The values of pulsation parameters and their error margins derived from the downward period spacing patterns for the multivariate analysis (see Section 5).

\begin{tabular}{|c|c|c|c|c|c|c|c|c|c|c|c|c|c|c|}
\hline KIC & $\langle P\rangle$ [days] & $\sigma_{P}$ & $\langle\Delta P\rangle$ [days] & $\sigma_{\Delta P}$ & $\left\langle\frac{\mathrm{d} \Delta P}{\mathrm{~d} P}\right\rangle$ & $\sigma_{\frac{d \Delta}{d} P}$ & $f_{g}\left[d^{-1}\right]$ & $\sigma_{f_{s}}$ & $A_{g}[\mathrm{mmag}]$ & $\sigma_{A_{g}}$ & $\Delta_{r e s}$ & $\sigma_{\Delta_{\text {res }}}$ & $P_{\max }[$ days] & $\sigma_{P_{\max }}$ \\
\hline 94 & 59 & 106 & 05 & 29 & 025 & 0.006 & 536 & 0 & 6.16 & 0.71 & 0.81 & 2.02 & & 0.00017 \\
\hline 3448365 & & 1 & & & -0.017 & 0.026 & & & & & 0.45 & & & \\
\hline 4846809 & 0973 & 00197 & & 87 & -0.010 & 0.006 & 1324 & 4 & & 29 & 1.82 & & & \\
\hline 5114382 & 670536 & 0.000096 & $520+2$ & 8 & -0.025 & 0.022 & 5265 & 06 & & 53 & 0.89 & 5.39 & 33 & 0014 \\
\hline 5522154 & 0.352971 & 0.000009 & 0.003819 & 0.000008 & -0.052 & 0.010 & 3.00986 & 0.00002 & 1. & 0.10 & 0.31 & 0.15 & 57 & 0.00002 \\
\hline 5708550 & 0.832343 & 0.000123 & & 0.000158 & -0.022 & 0.003 & 1.11550 & & & 0.39 & 1.30 & 2.12 & & 015 \\
\hline 5788623 & 1.133749 & 0.000 & 0.012 & 90 & -0.015 & 0.002 & 0.77895 & 0.00 & 9. & 1.84 & 1.97 & & & \\
\hline 6468146 & 0.6 & 0.000056 & 96 & 0.000084 & -0.035 & 0.008 & 70 & 0.00 & & 0.04 & 1.18 & 0.85 & & 12 \\
\hline 6468987 & & 58 & & 05 & -0.038 & 0.017 & 9 & 0.0 & & 8 & 0.19 & 0.76 & & \\
\hline 6678174 & 67 & 0.000 & & 71 & -0.015 & 0.542 & 77 & 0.00 & & 0.59 & 3.02 & 55.65 & & \\
\hline 6935014 & & 0.000104 & & & -0.025 & 0.005 & & 0.00 & & 0.79 & 1.88 & 3.21 & & 030 \\
\hline 6953103 & 58 & 0.000 & & 48 & -0.031 & 0.004 & & 0. & & 3.23 & 1.44 & 1. & & \\
\hline 7023122 & 0.479 & 0.000024 & 0.0 & 0.000 & -0.048 & 0.034 & 1.87611 & 0.0 & 13.90 & 0.40 & 1.27 & 0.43 & & 0. \\
\hline 7365537 & 344913 & 0.000007 & 0.00 & 0.000008 & -0.049 & 0.011 & 563 & 0.00 & & 0.23 & 0.00 & 0 . & & 0.00001 \\
\hline 7380501 & 65164 & 0.000137 & & & -0.017 & 0.003 & 0.96329 & 0.00 & & 0 & 1.12 & 4. & & 24 \\
\hline 7434470 & 94 & 3 & & 3 & -0.040 & 0.001 & 1. & 0.0 & & 0 & 1.26 & 0 . & & \\
\hline 7583 & 0.6 & 0.000 & 0 & 0.00 & -0.031 & 0.007 & 74 & 0.00003 & 6.28 & 0.54 & 0.90 & 1.44 & 27 & 0.00007 \\
\hline 7939065 & & 0.000037 & & 0.000 & -0.036 & 0.002 & & 0.00 & & 0.73 & 0.22 & 0.08 & & 014 \\
\hline 8364249 & 0.522644 & 0.000017 & 0.002100 & 0.000019 & -0.029 & 0.003 & 1.86938 & 0.00002 & 3.94 & 0.17 & 0.57 & 0.96 & & 0.00003 \\
\hline 8375138 & 0.469786 & 0.000023 & 0.002377 & 0.000025 & -0.034 & 0.007 & 2.07777 & 0.00002 & & 0.31 & 0.52 & 0.91 & .51310 & 0.00003 \\
\hline 8645874 & & 0.000019 & & 0.000029 & -0.016 & 0.005 & 1.84701 & 0.00001 & & 0.17 & 1.15 & 0.06 & & 0.00017 \\
\hline 8836473 & 9 & 0.000035 & 0 & 0.00 & -0.034 & 0.061 & 1.8 & $0.0 c$ & 1. & 0.13 & 0.44 & 0. & & 0.0 \\
\hline 9419694 & 0.937988 & 0.000193 & 0.0 & 0.000 & -0.016 & 0.023 & 1.03279 & 0.00004 & 14.7 & 1.51 & 2.02 & 3.78 & 79 & 0.00037 \\
\hline 9480469 & 0.518274 & 0.000039 & 0.002455 & 0.000033 & -0.028 & 0.035 & 1.99485 & 0.00003 & 7.14 & 0.66 & 0.23 & 3.80 & .54748 & 0.00006 \\
\hline 9595743 & 0.683181 & 0.000099 & 0.006987 & 0.000146 & -0.033 & 0.040 & 1.72911 & 0.00003 & 6.93 & 0.62 & 1.09 & 2.68 & .76882 & 0.00014 \\
\hline 9751996 & 0.85 & 0.000051 & 0 & 0.000 & 0.002 & 0.010 & 1.28331 & 0.00006 & 2. & 0.36 & 2.68 & 0.50 & & 0.00010 \\
\hline 10224094 & 0.875860 & 0.000110 & & 34 & -0.017 & 0.010 & & 0.00 & 2. & & 0.00 & 1. & & 0.00031 \\
\hline & 0.996770 & & & & -0.018 & 0.061 & 0.95498 & & & 0.36 & 1.09 & 28.11 & & 0.00018 \\
\hline 11080103 & 0.911278 & 0.000154 & 0.011062 & 0.000194 & -0.019 & 0.016 & 1.24139 & 0.00002 & 12.13 & 0.75 & 0.97 & 4.74 & 1.09122 & 0.00017 \\
\hline
\end{tabular}


Table A4-Continued

\begin{tabular}{ccccccccccccccc}
\hline \hline KIC & $\langle P\rangle$ [days] & $\sigma_{P}$ & $\langle\Delta P\rangle$ days $]$ & $\sigma_{\Delta P}$ & $\left\langle\frac{\mathrm{d} \Delta P}{\mathrm{~d} P}\right\rangle$ & $\sigma_{\frac{\mathrm{d} P}{\mathrm{~d} P}}$ & $f_{g}\left[d^{-1}\right]$ & $\sigma_{f_{g}}$ & $A_{g}[\mathrm{mmag}]$ & $\sigma_{A_{g}}$ & $\Delta_{\text {res }}$ & $\sigma_{\Delta_{\text {res }}}$ & $P_{\max }[\mathrm{days}]$ & $\sigma_{P_{\max }}$ \\
\hline 11099031 & 0.698765 & 0.000055 & 0.004375 & 0.000067 & -0.026 & 0.003 & 0.91665 & 0.00003 & 1.42 & 0.13 & 0.97 & 0.99 & 0.81286 & 0.00006 \\
11294808 & 0.912759 & 0.000098 & 0.006943 & 0.000118 & -0.043 & 0.023 & 2.22475 & 0.00004 & 2.32 & 0.22 & 1.17 & 13.74 & 0.99168 & 0.00013 \\
11456474 & 0.690040 & 0.000060 & 0.003209 & 0.000071 & -0.025 & 0.002 & 1.47147 & 0.00002 & 4.17 & 0.26 & 1.19 & 1.38 & 0.78283 & 0.00007 \\
11721304 & 1.026382 & 0.000331 & 0.011981 & 0.000430 & -0.020 & 0.002 & 0.92287 & 0.00009 & 4.10 & 1.03 & 2.42 & 0.77 & 1.32327 & 0.00053 \\
11754232 & 0.665339 & 0.000034 & 0.031646 & 0.000051 & -0.002 & 0.034 & 1.10338 & 0.00006 & 2.03 & 0.30 & 1.94 & 0.37 & 0.90630 & 0.00005 \\
11826272 & 1.074839 & 0.000399 & 0.015434 & 0.000543 & -0.018 & 0.001 & 0.83370 & 0.00006 & 11.61 & 1.94 & 1.87 & 0.50 & 1.59754 & 0.00086 \\
11907454 & 0.548784 & 0.000061 & 0.002847 & 0.000084 & -0.033 & 0.006 & 1.18715 & 0.00003 & 4.43 & 0.36 & 0.87 & 0.89 & 0.60608 & 0.00007 \\
11917550 & 0.716579 & 0.000084 & 0.005704 & 0.000104 & -0.028 & 0.003 & 1.28768 & 0.00004 & 9.21 & 0.87 & 1.86 & 1.08 & 0.82445 & 0.00018 \\
11920505 & 0.787720 & 0.000142 & 0.007948 & 0.000144 & -0.027 & 0.003 & 1.19884 & 0.00004 & 13.26 & 1.43 & 1.90 & 1.58 & 0.95371 & 0.00020 \\
12066947 & 0.359580 & 0.000016 & 0.002396 & 0.000017 & -0.040 & 0.003 & 2.72379 & 0.00004 & 2.77 & 0.30 & 0.17 & 0.14 & 0.40962 & 0.00003 \\
12458189 & 0.917404 & 0.000135 & 0.005263 & 0.000178 & -0.019 & 0.003 & 1.03961 & 0.00003 & 4.44 & 0.30 & 1.09 & 2.24 & 1.12169 & 0.00019 \\
\hline
\end{tabular}




\section{A. Detected period spacing patterns}

Below we provide figures of all the detected period spacing patterns for the sample stars. The used symbols are the same as the ones used in Fig. 3 of the main text.
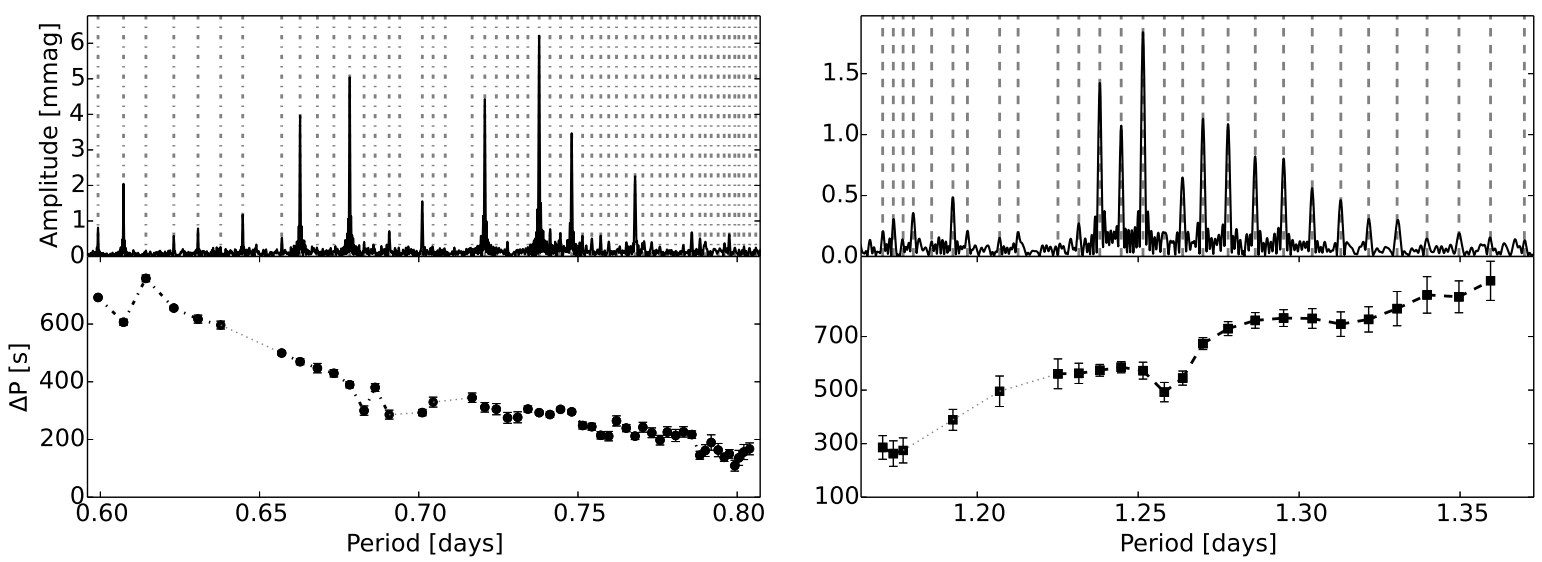

Fig. 12.— The period spacing patterns of KIC 2710594.
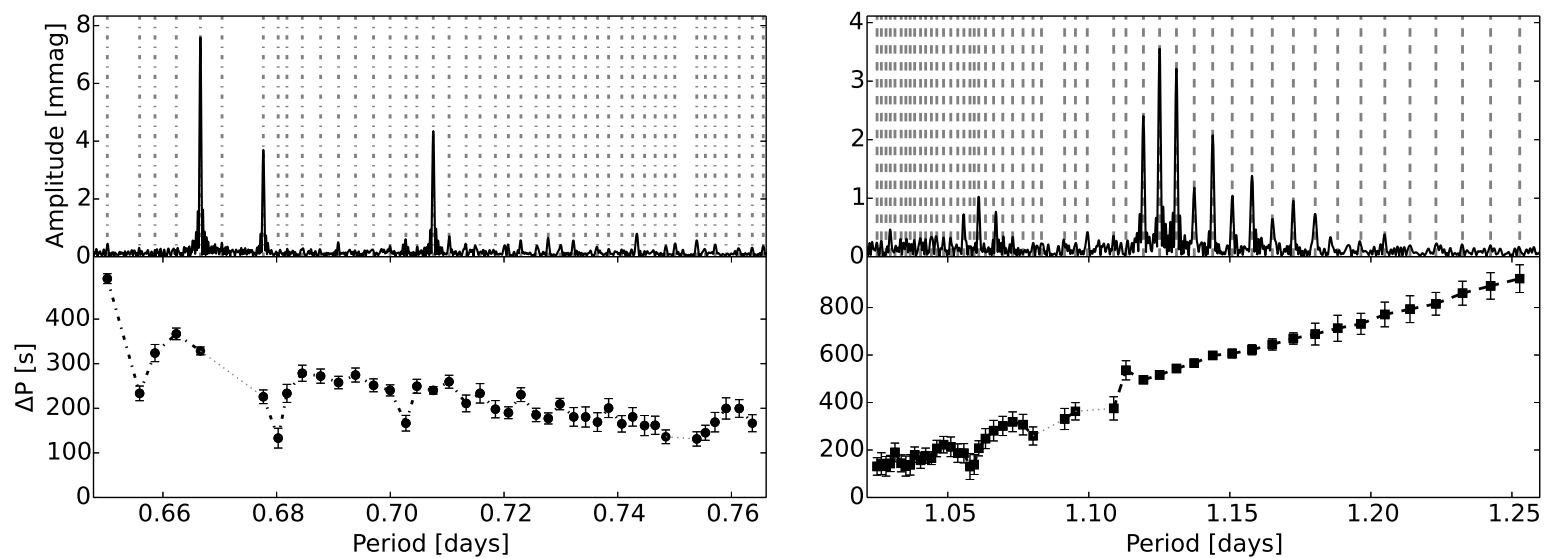

Fig. 13.- The period spacing patterns of KIC 3448365. 


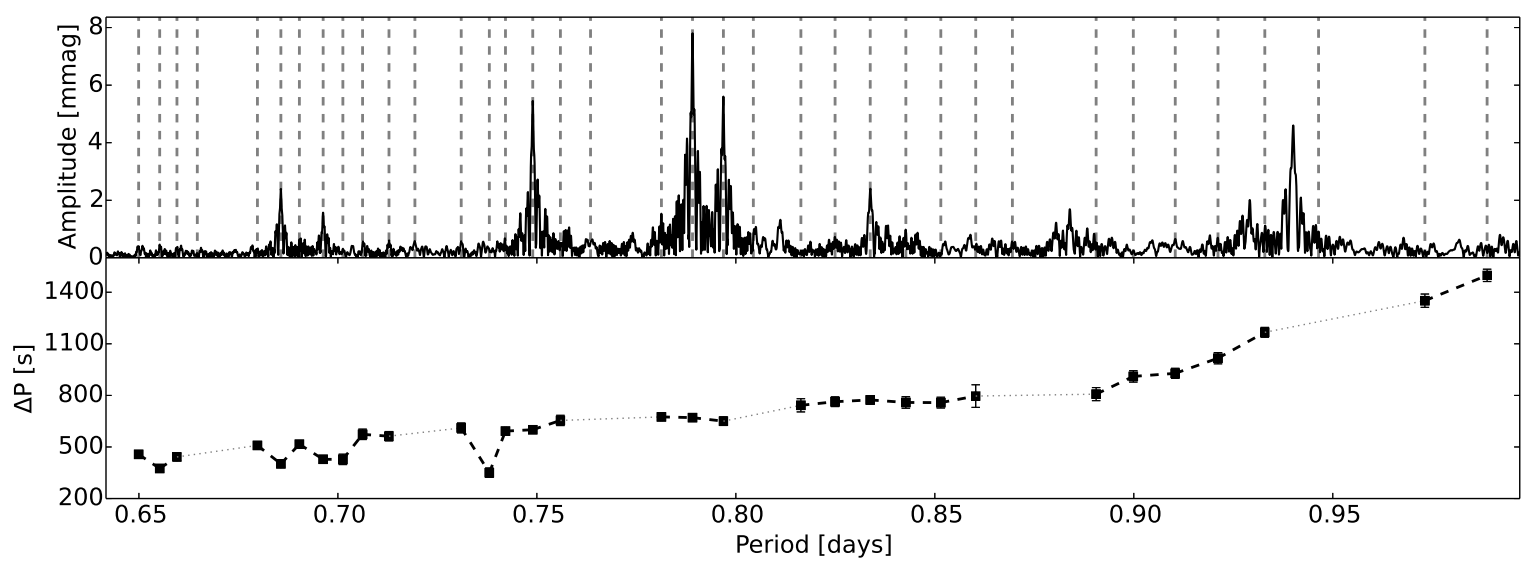

Fig. 14.- The period spacing patterns of KIC 4757184.
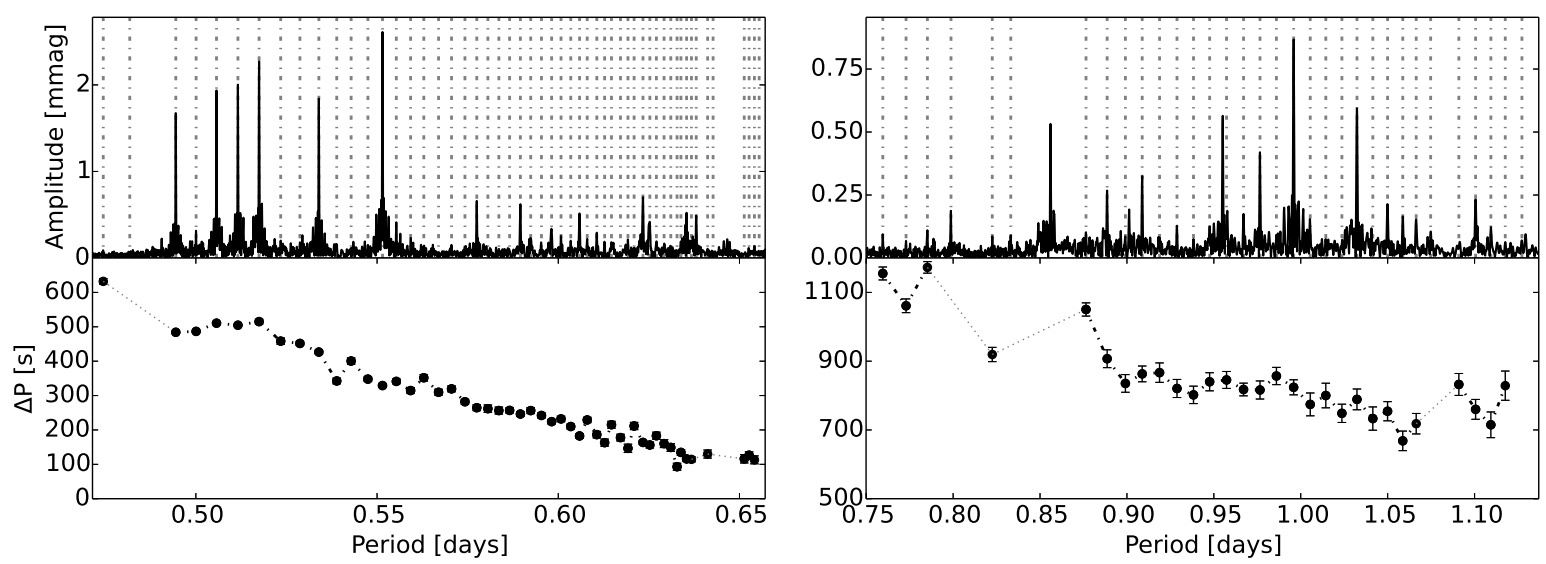

Fig. 15. - The period spacing patterns of KIC 4846809. 

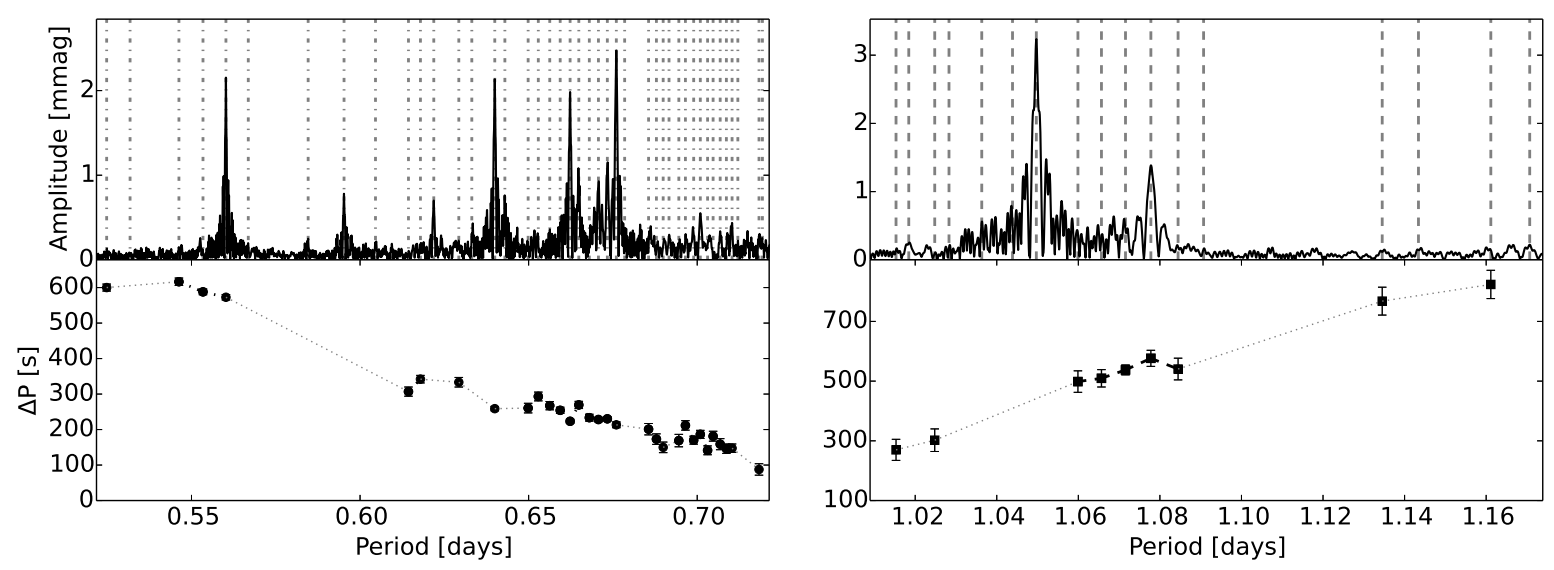

Fig. 16.- The period spacing patterns of KIC 5114382.

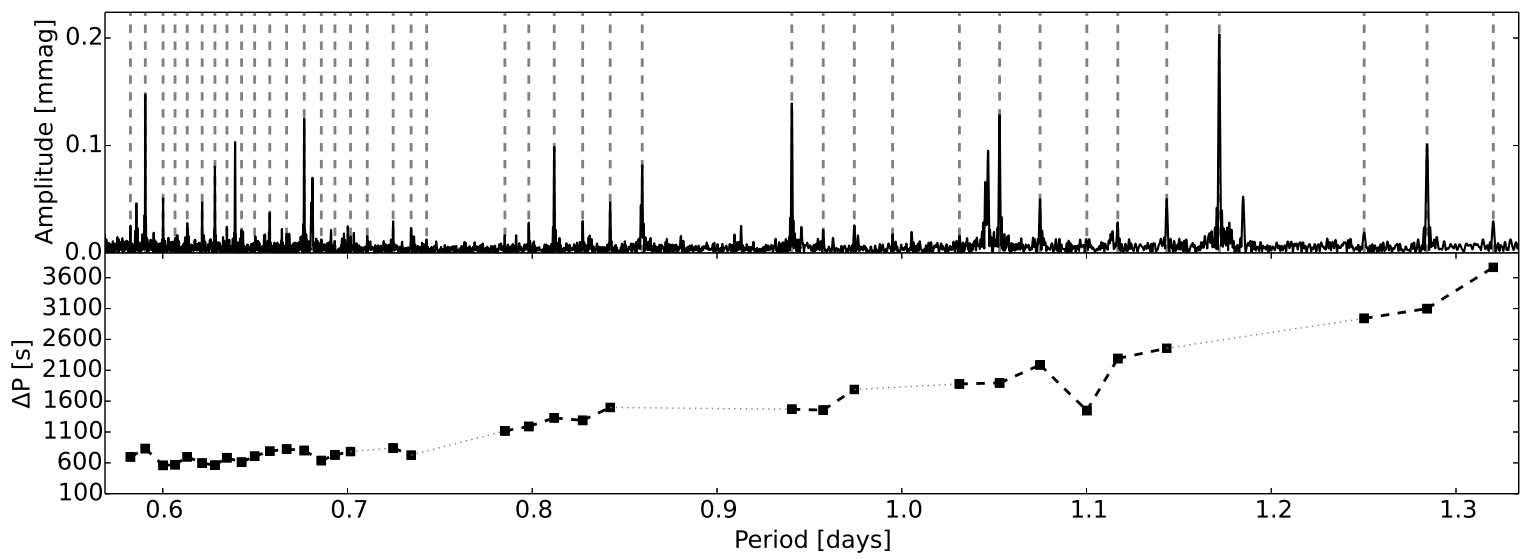

Fig. 17. - The period spacing patterns of KIC 5350598. 


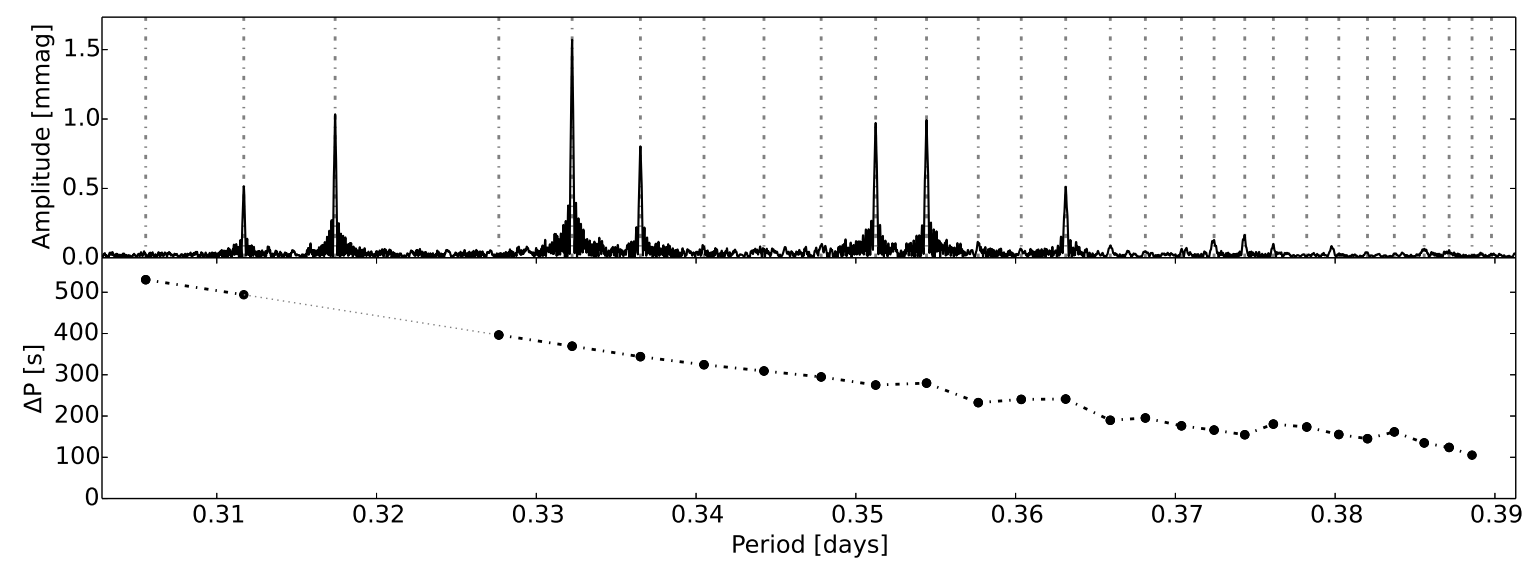

Fig. 18.- The period spacing patterns of KIC 5522154.

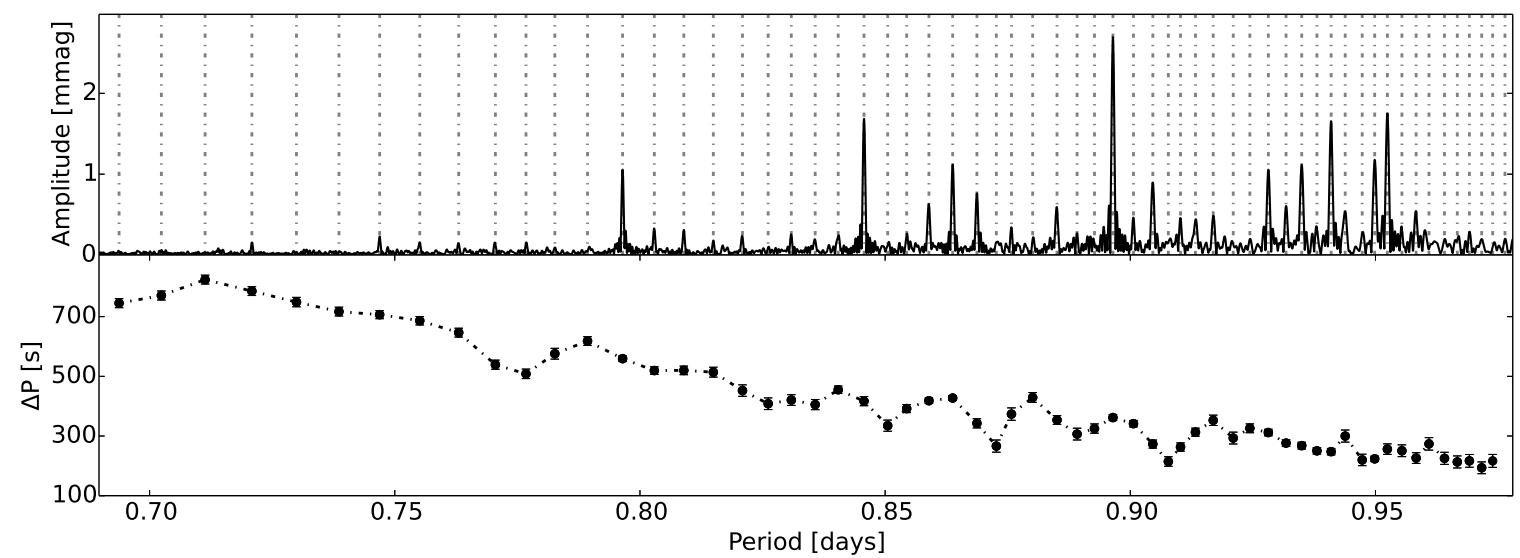

Fig. 19. - The period spacing patterns of KIC 5708550. 


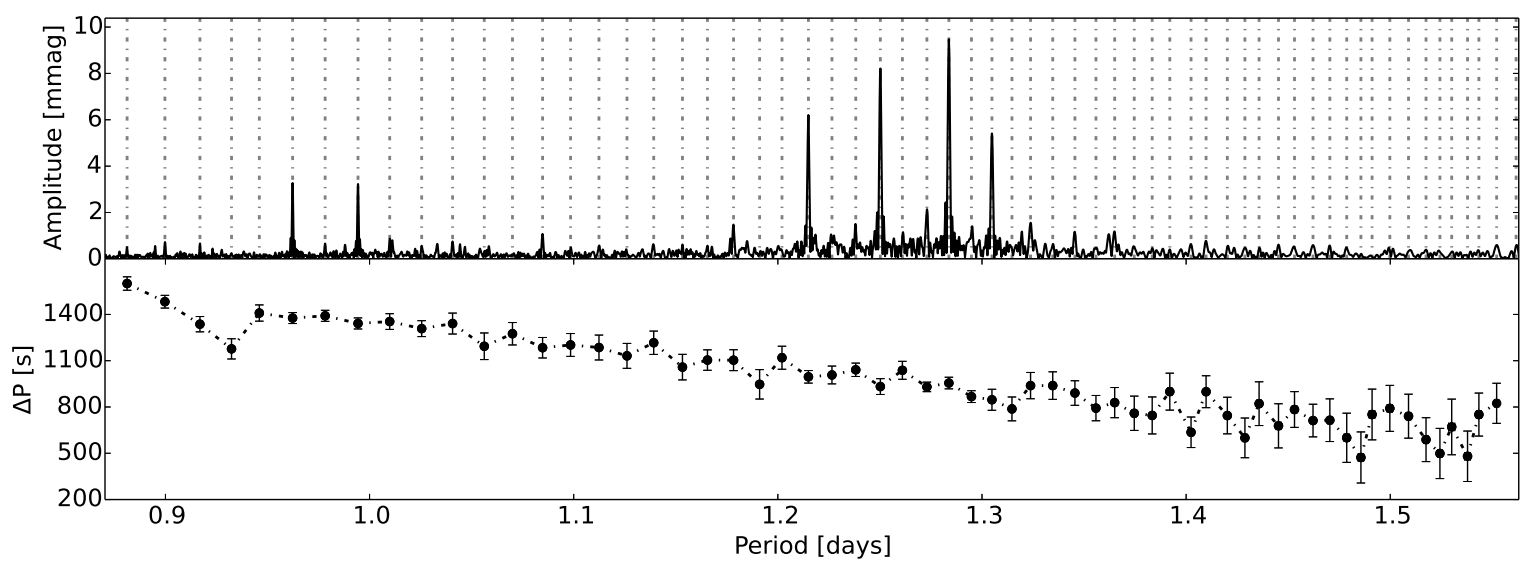

Fig. 20.- The period spacing patterns of KIC 5788623.

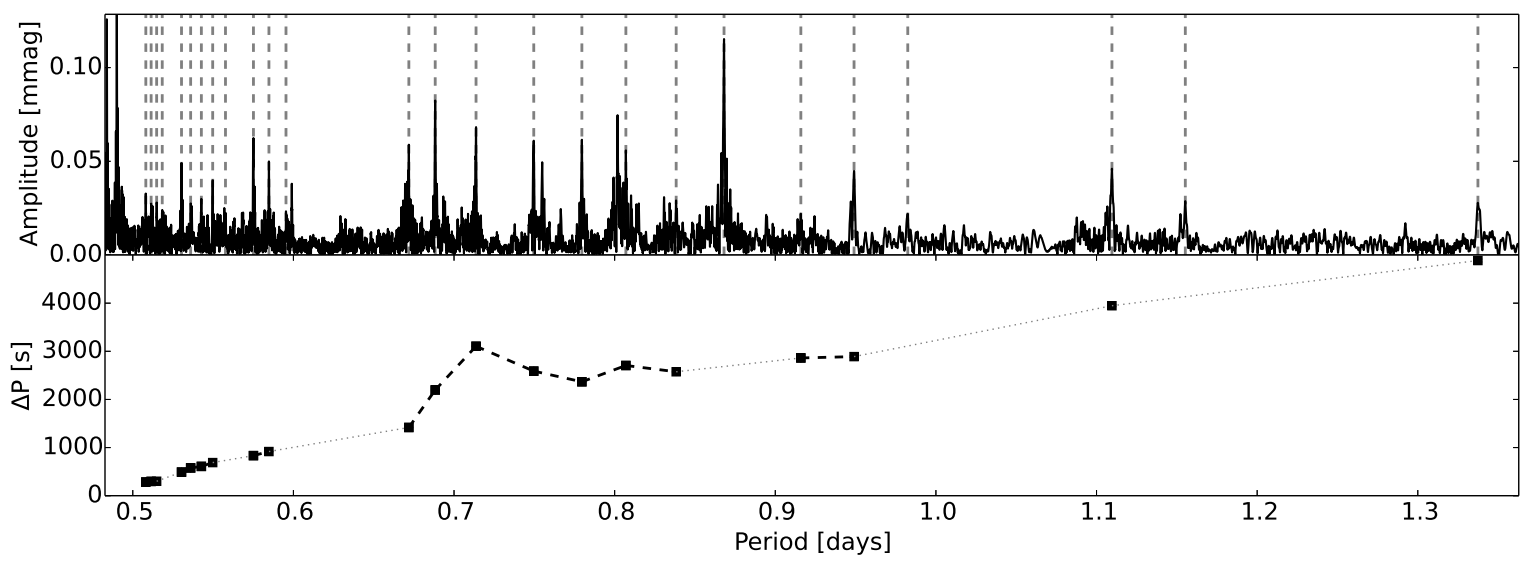

Fig. 21. - The period spacing patterns of KIC 6185513. 


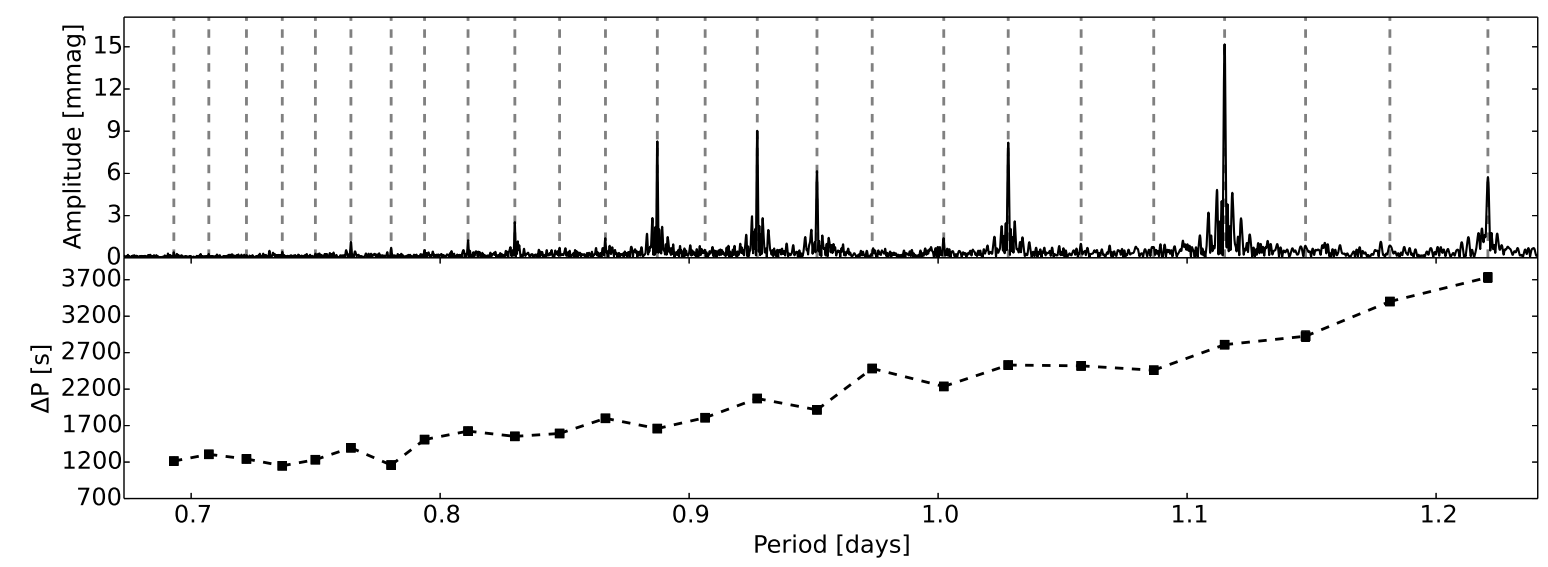

Fig. 22.- The period spacing patterns of KIC 6425437.

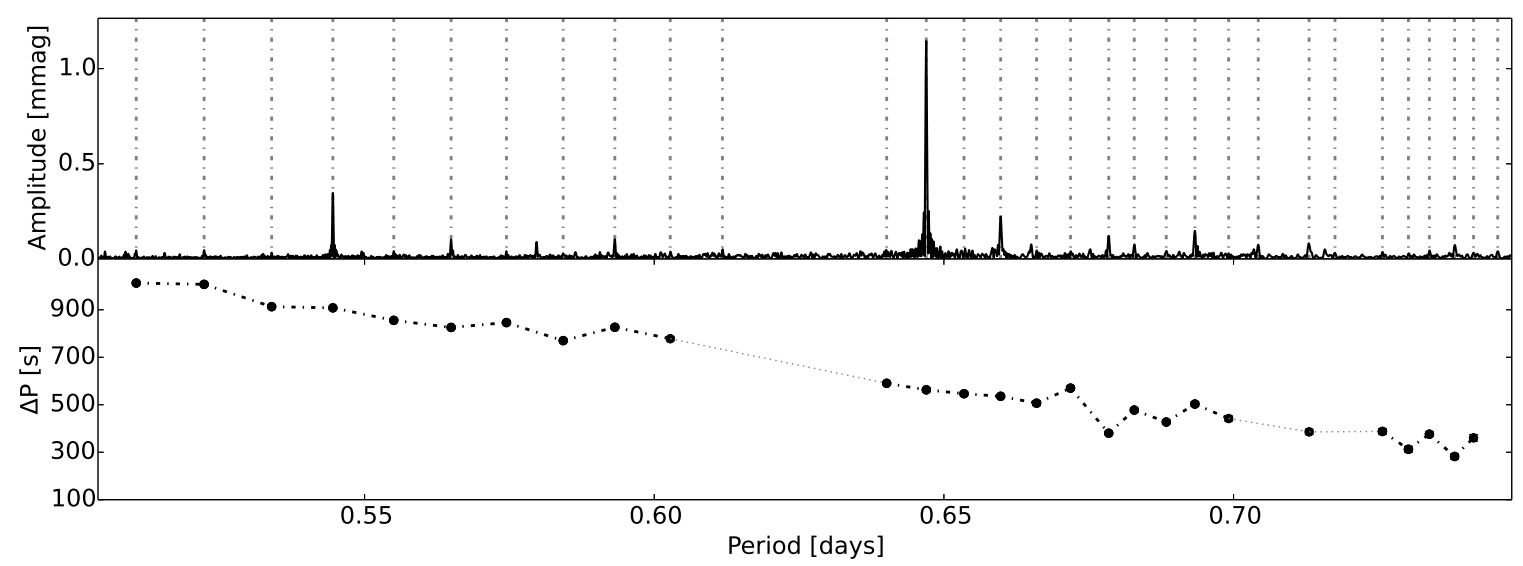

Fig. 23.- The period spacing patterns of KIC 6468146. 

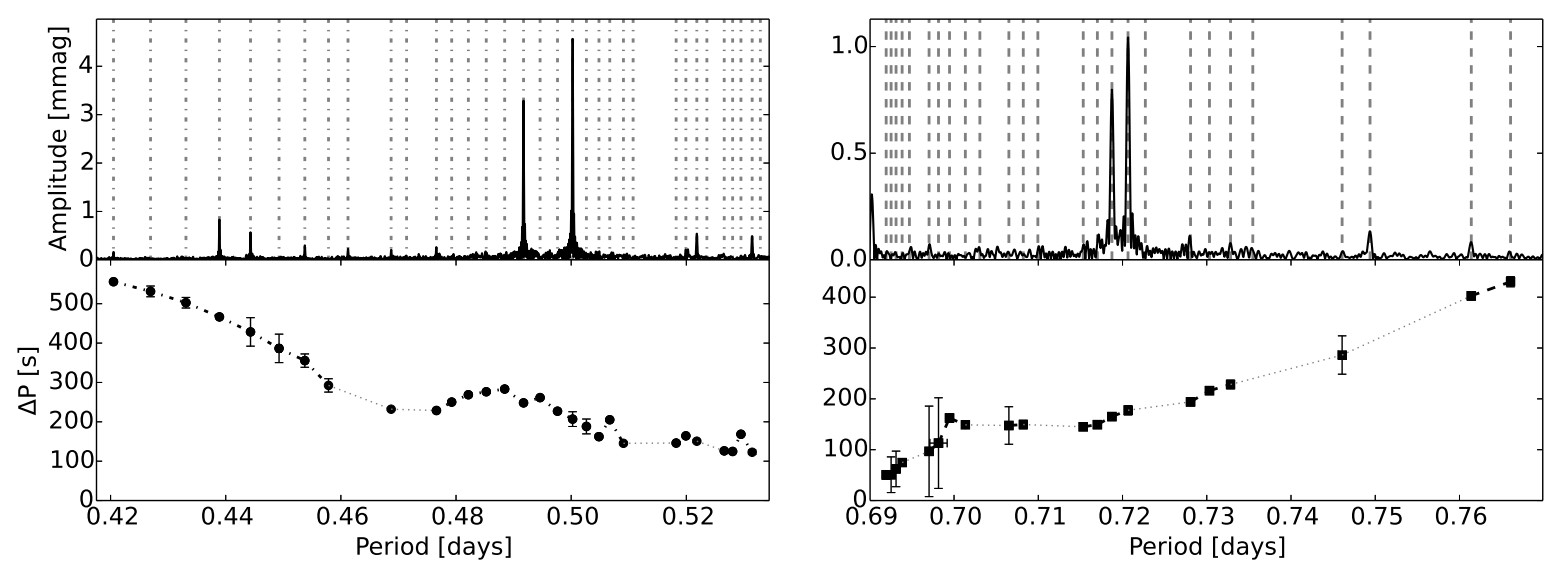

Fig. 24.- The period spacing patterns of KIC 6468987.

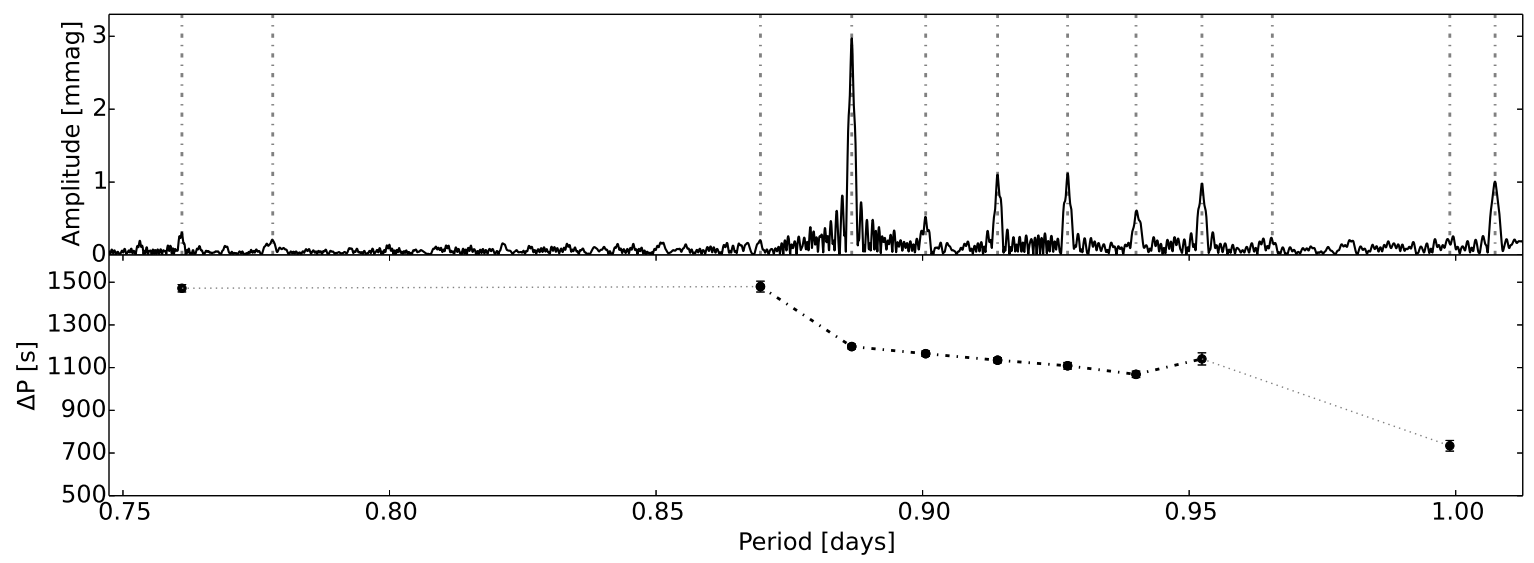

Fig. 25.- The period spacing patterns of KIC 6678174. 

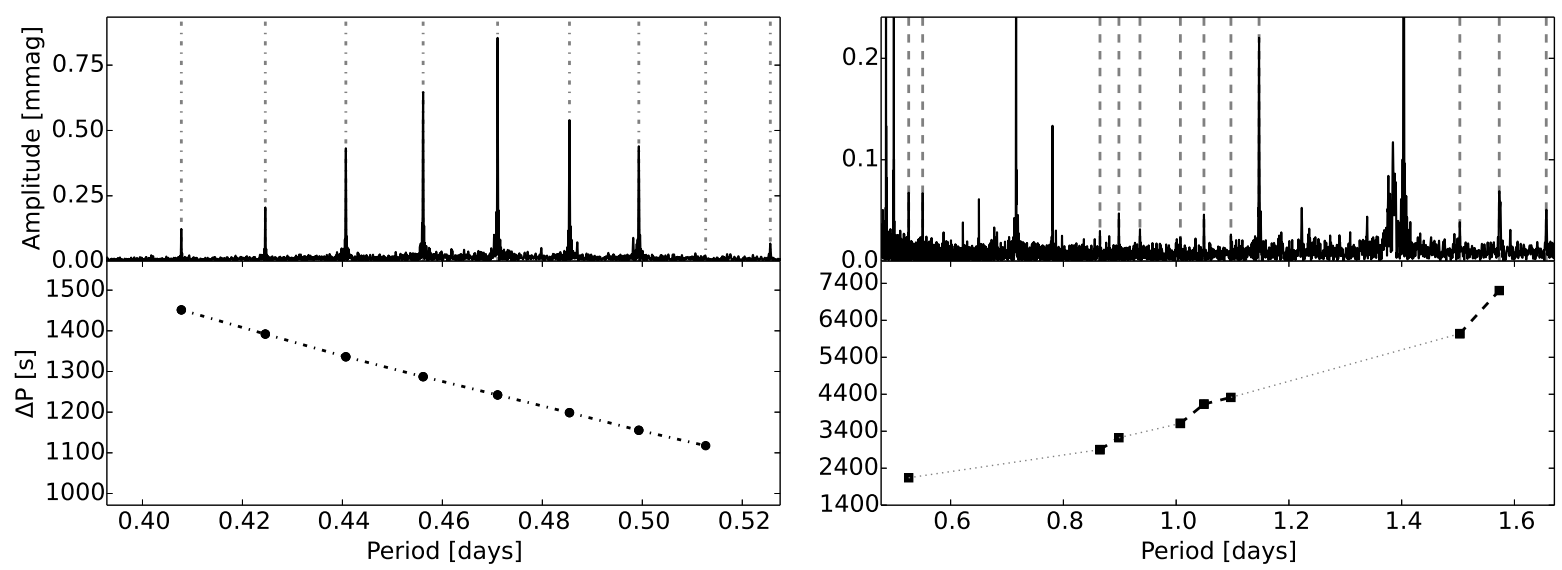

Fig. 26.- The period spacing patterns of KIC 6778063.

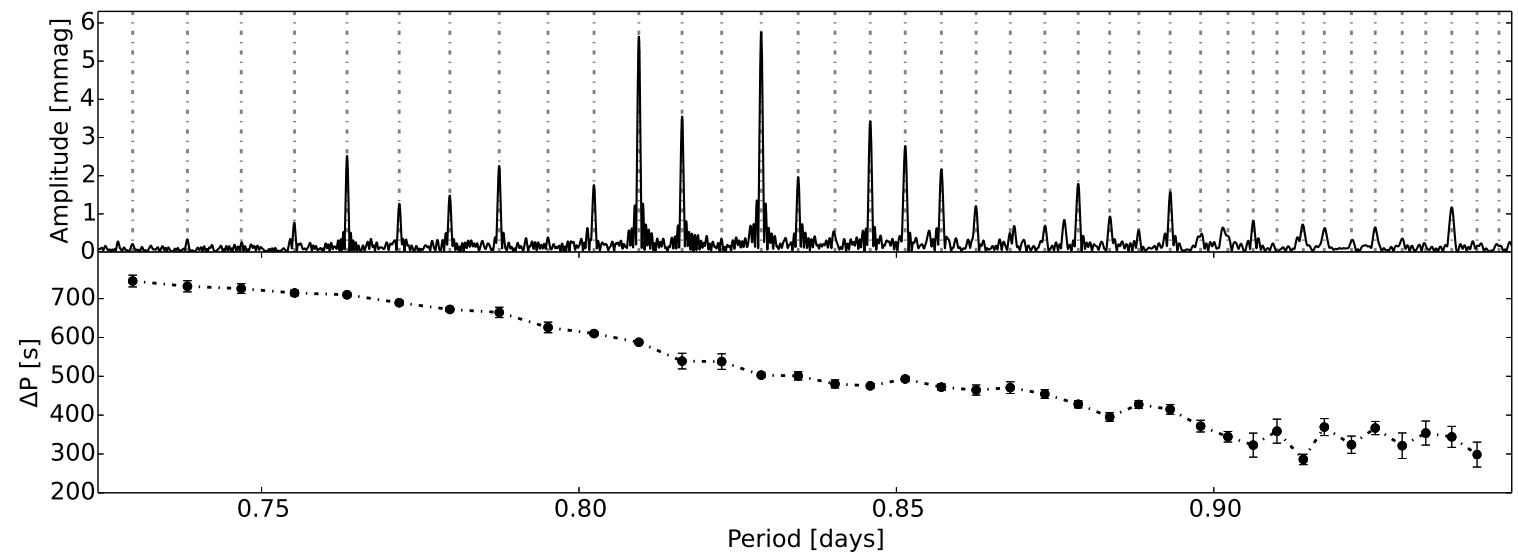

Fig. 27.- The period spacing patterns of KIC 6935014. 


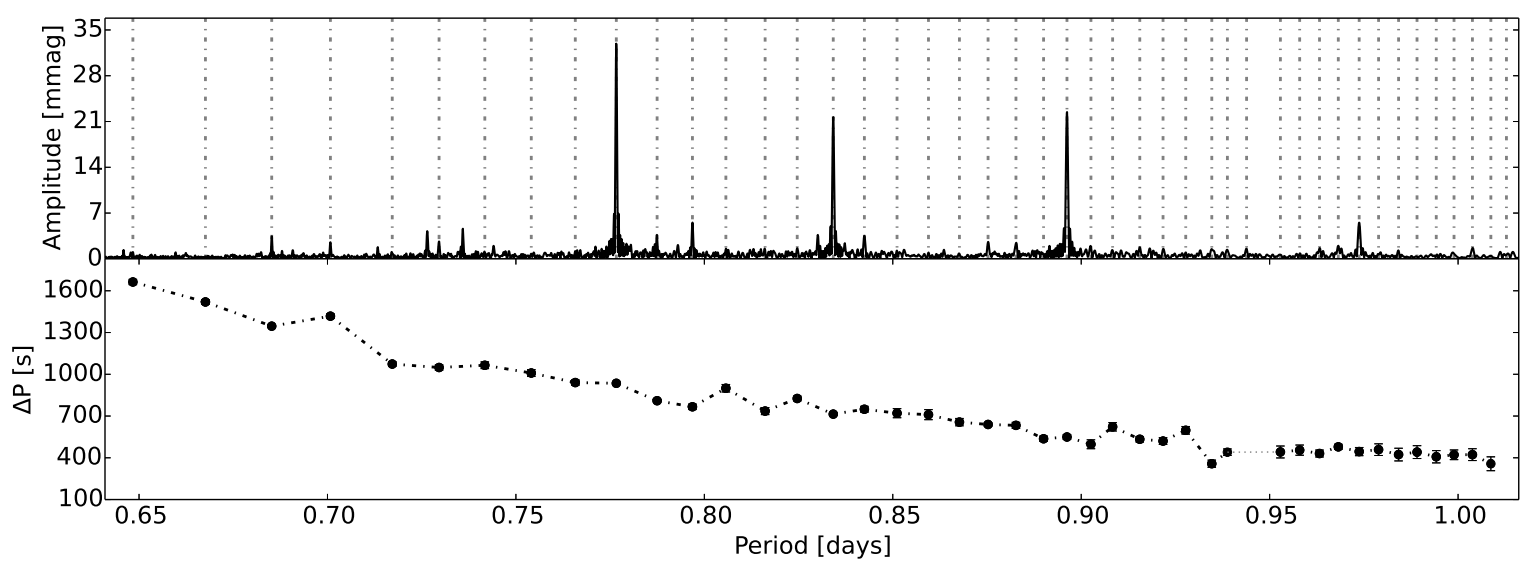

Fig. 28. - The period spacing patterns of KIC 6953103.

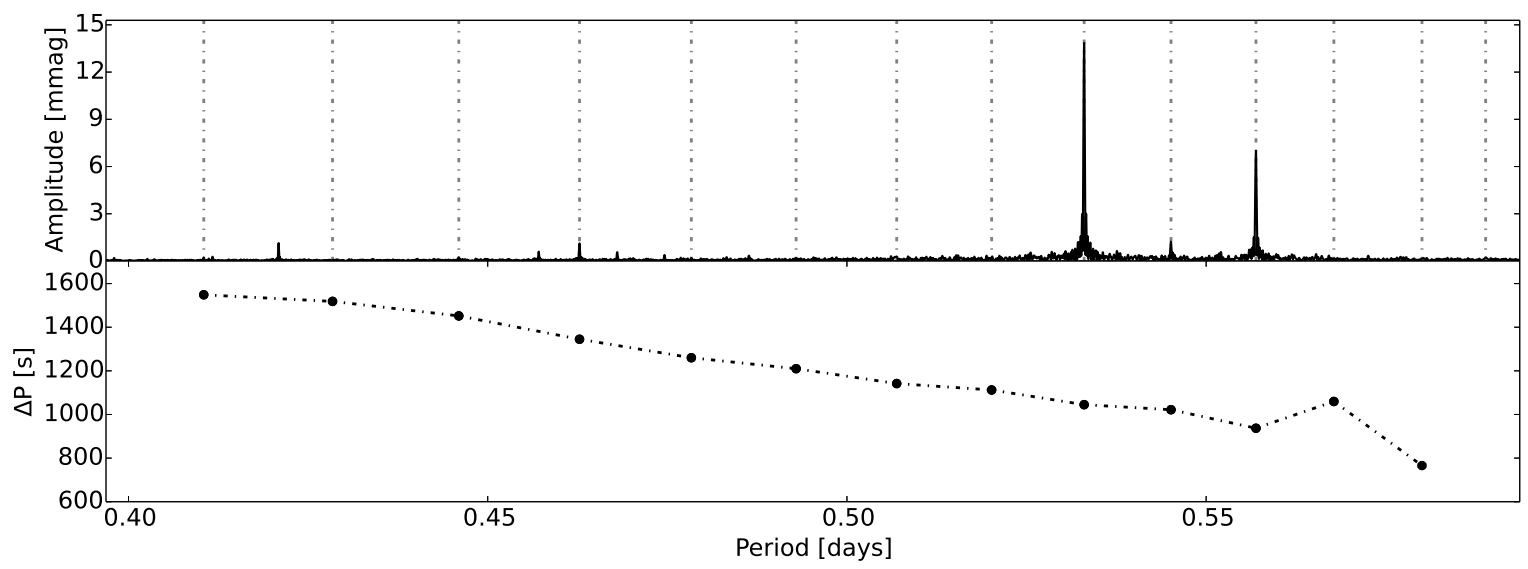

Fig. 29. - The period spacing patterns of KIC 7023122. 


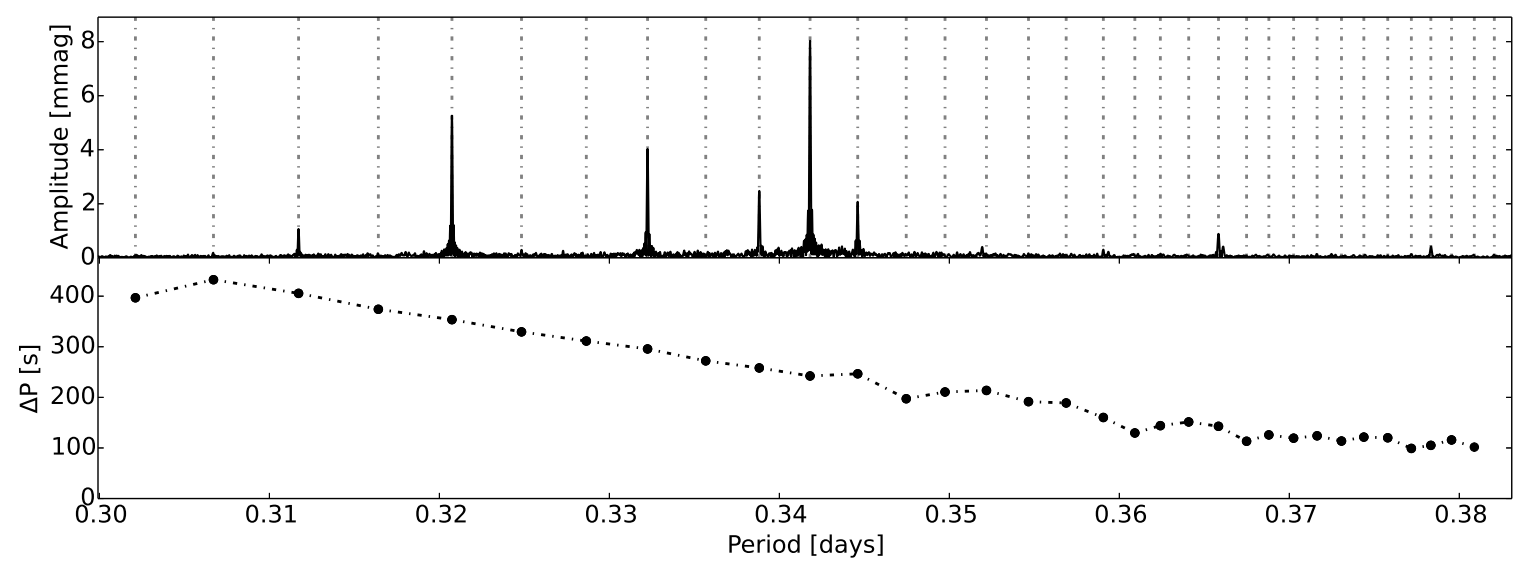

Fig. 30.- The period spacing patterns of KIC 7365537.

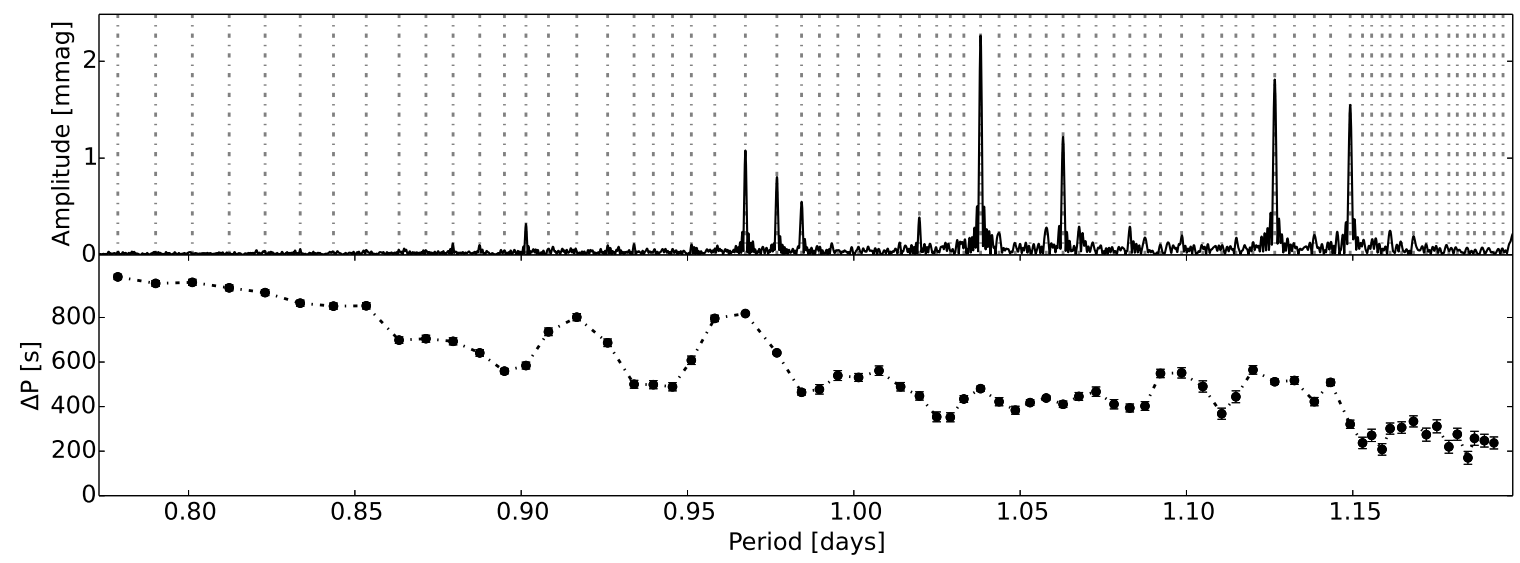

Fig. 31. - The period spacing patterns of KIC 7380501. 


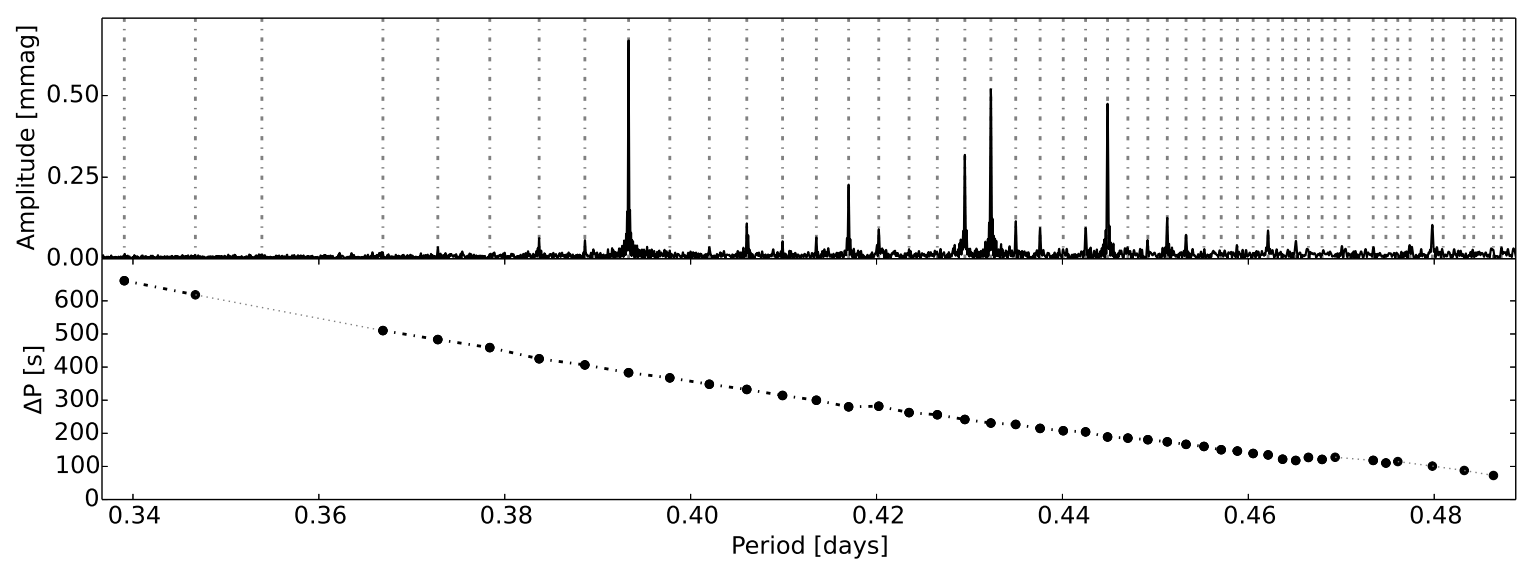

Fig. 32.- The period spacing patterns of KIC 7434470.
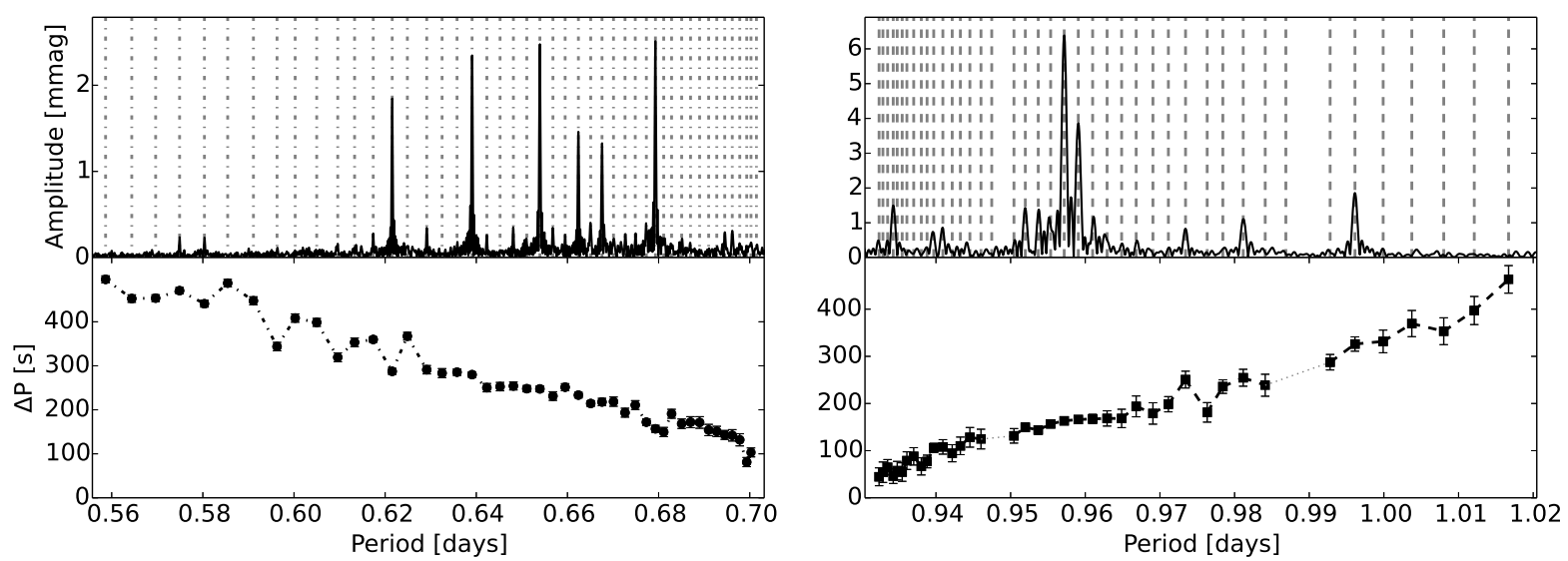

Fig. 33.- The period spacing patterns of KIC 7583663. 


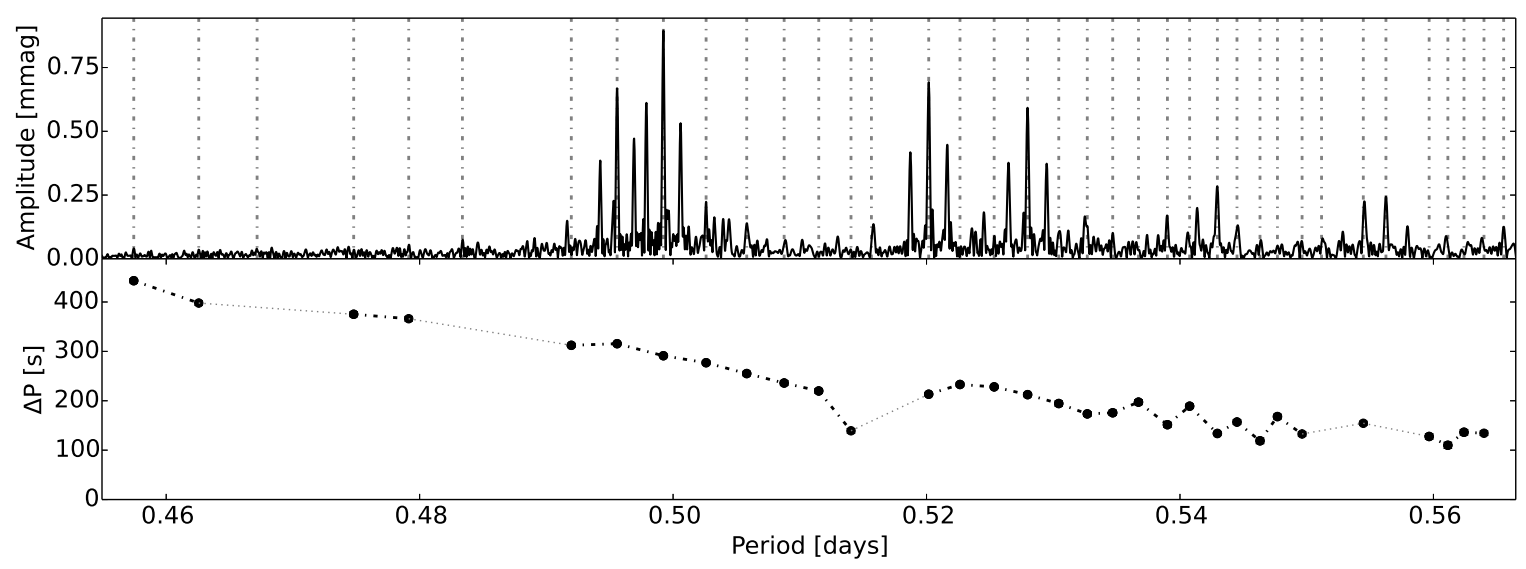

Fig. 34.- The period spacing patterns of KIC 7746984.

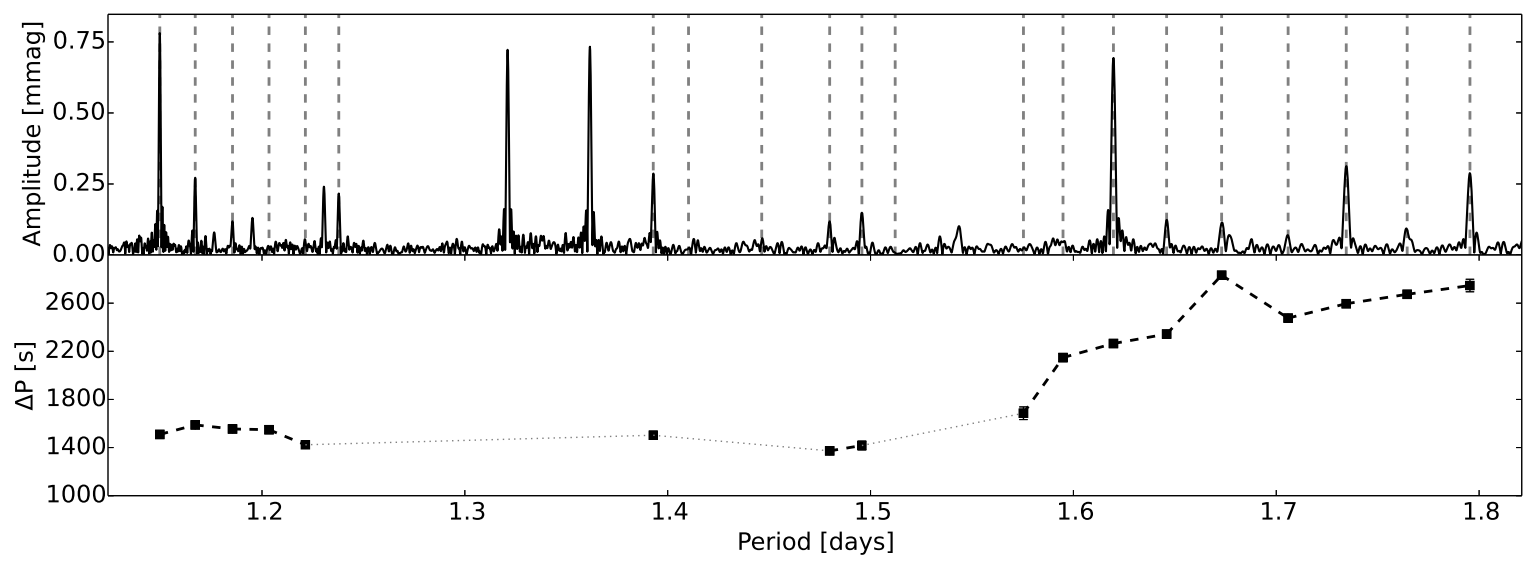

Fig. 35.- The period spacing patterns of KIC 7867348. 


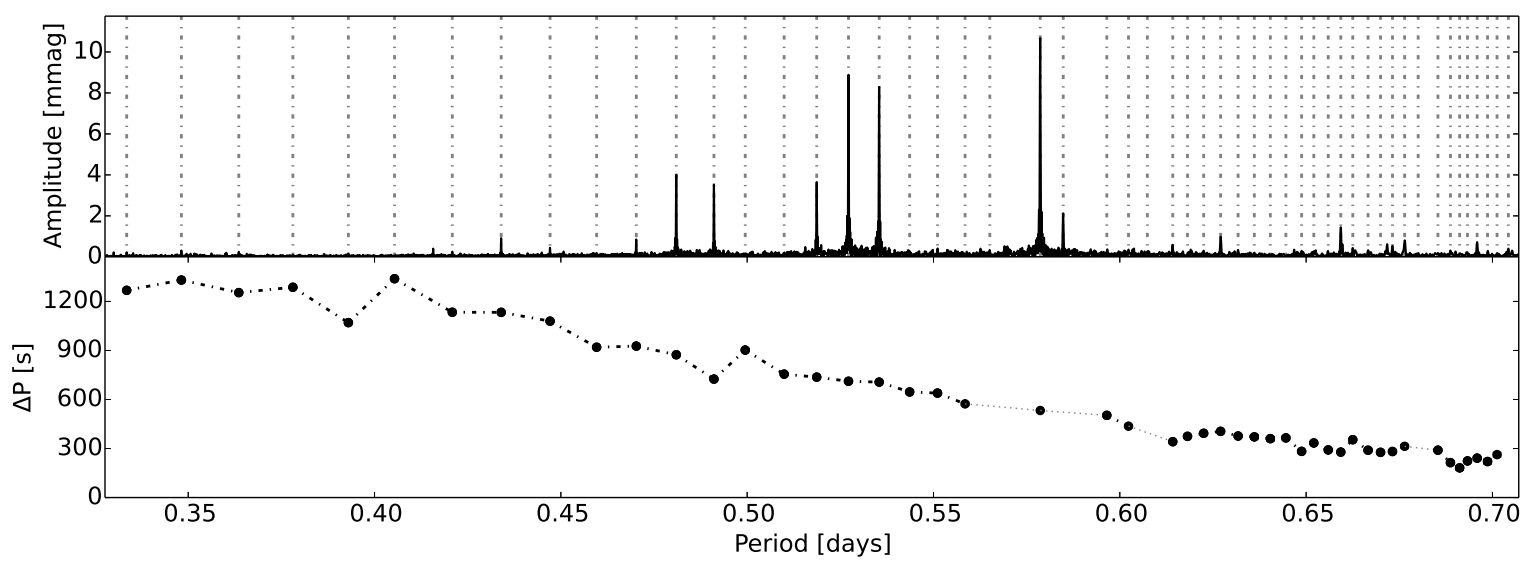

Fig. 36.- The period spacing patterns of KIC 7939065.

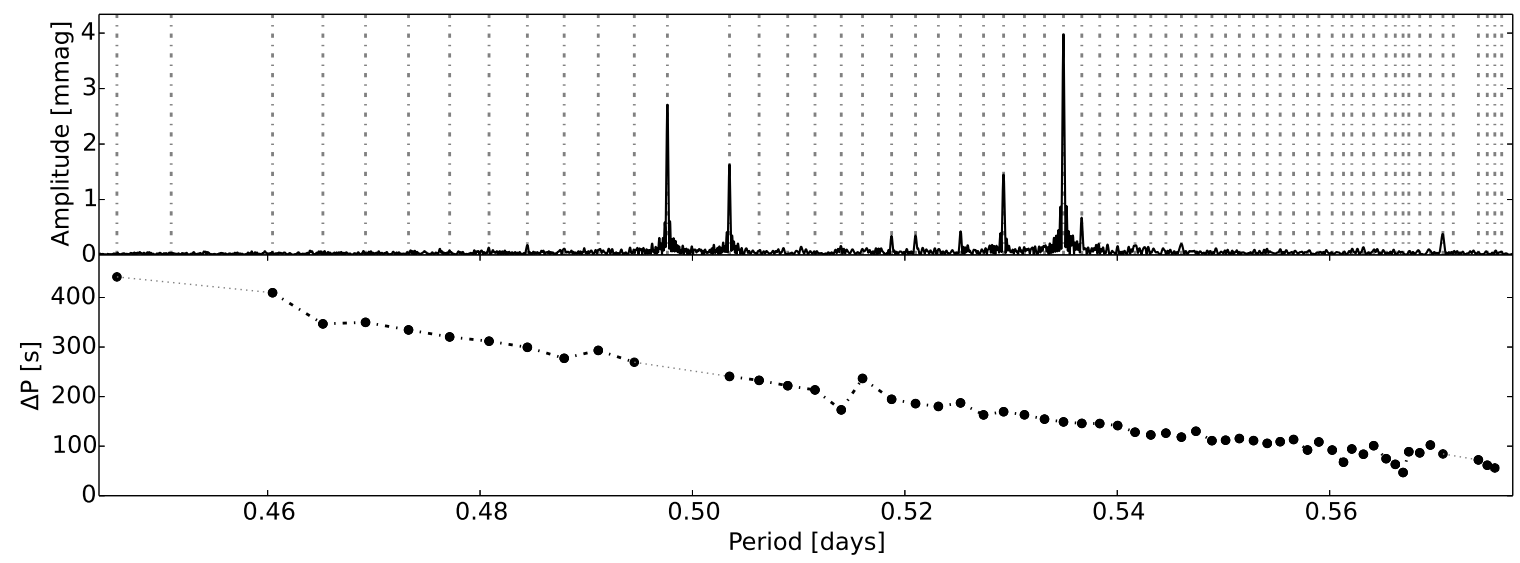

Fig. 37. - The period spacing patterns of KIC 8364249. 

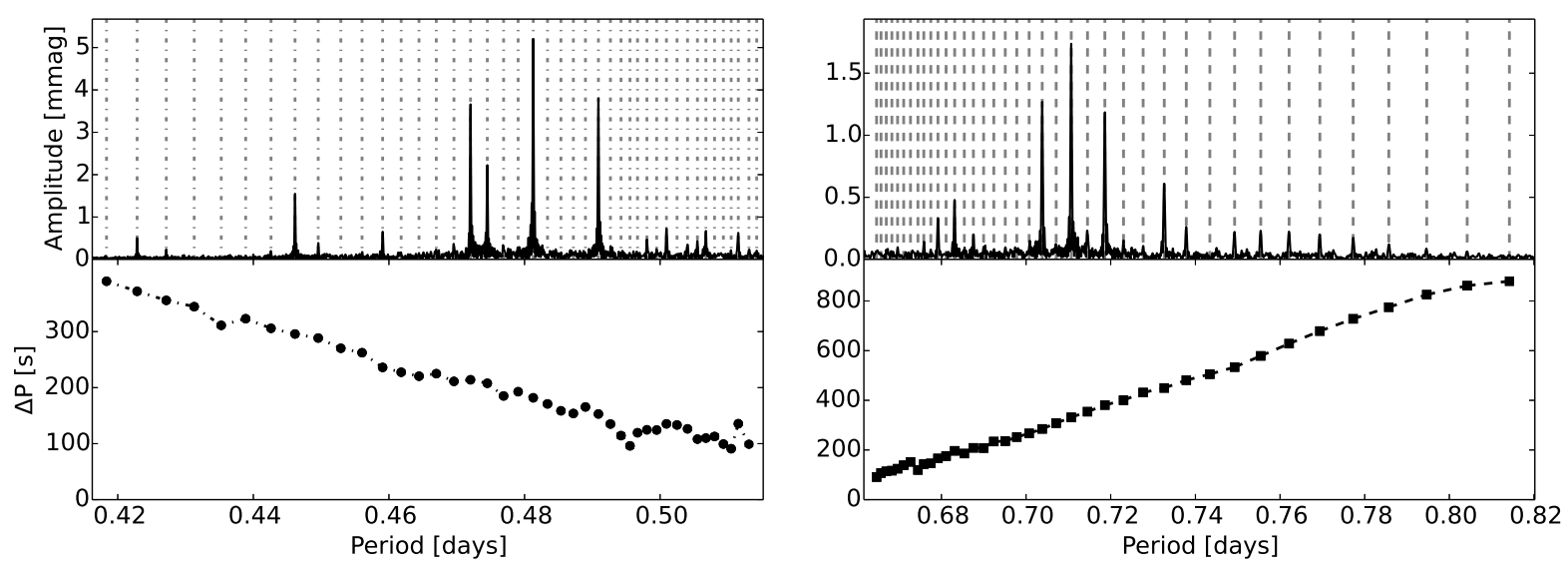

Fig. 38.- The period spacing patterns of KIC 8375138.

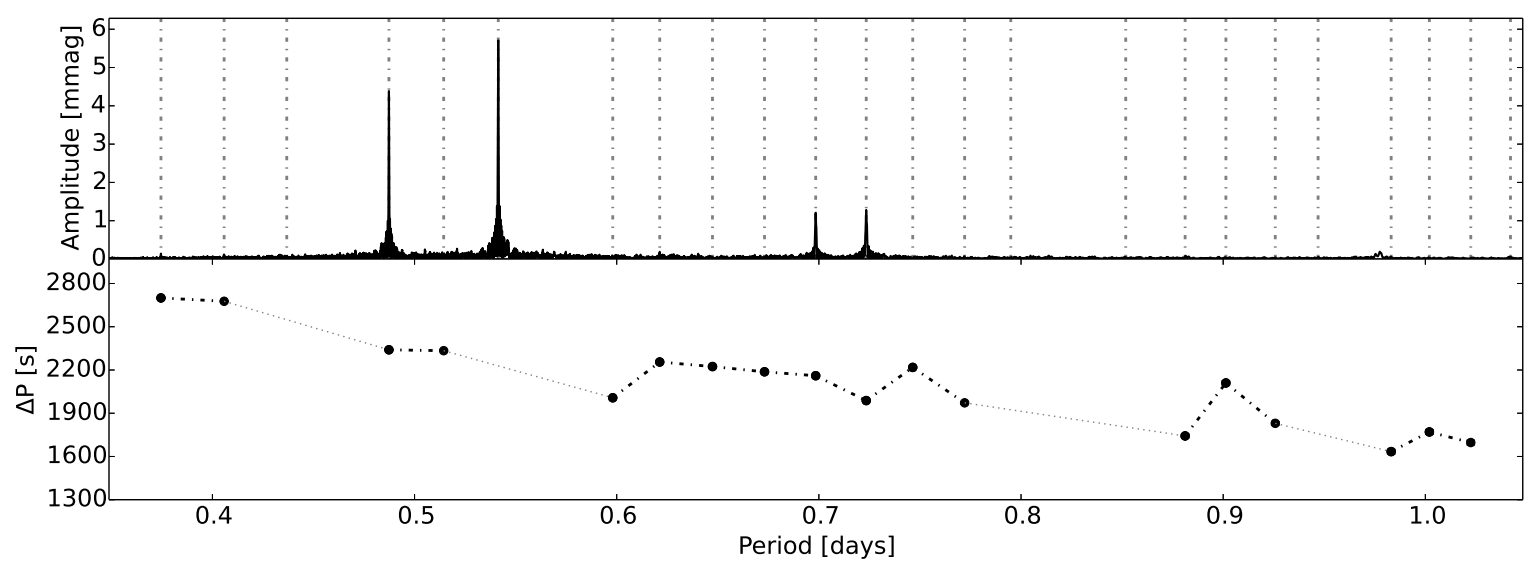

Fig. 39. - The period spacing patterns of KIC 8645874 . 


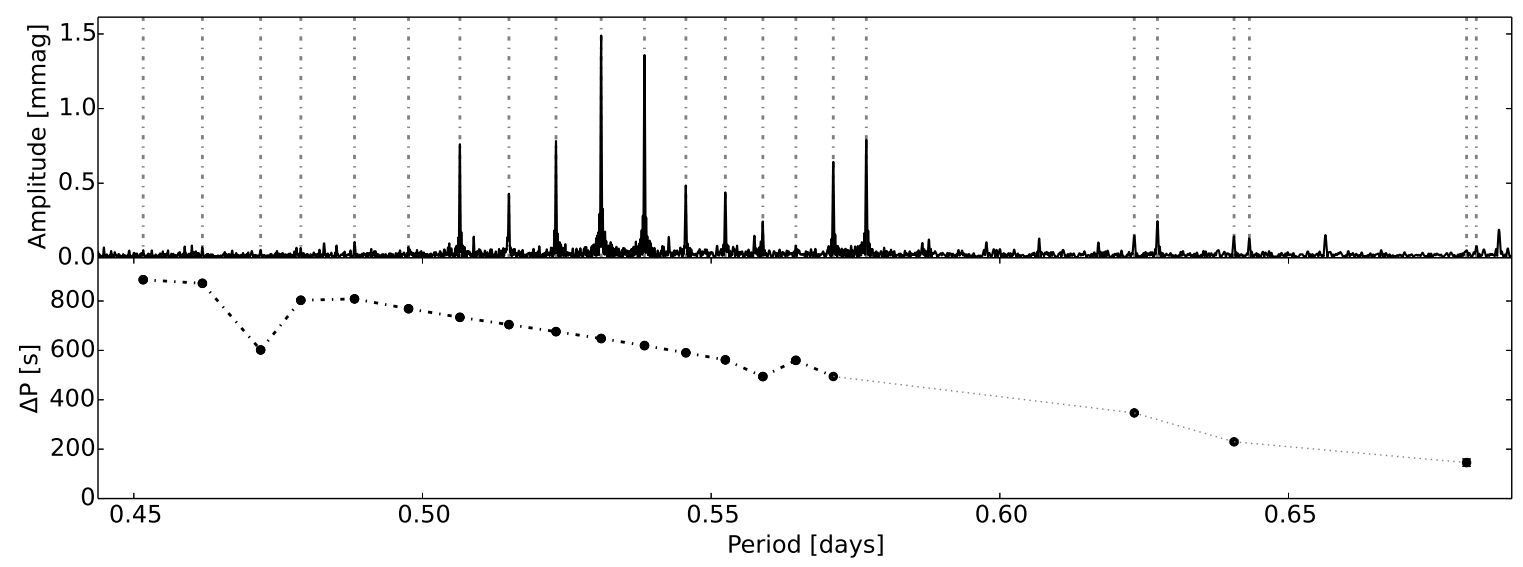

Fig. 40.- The period spacing patterns of KIC 8836473.
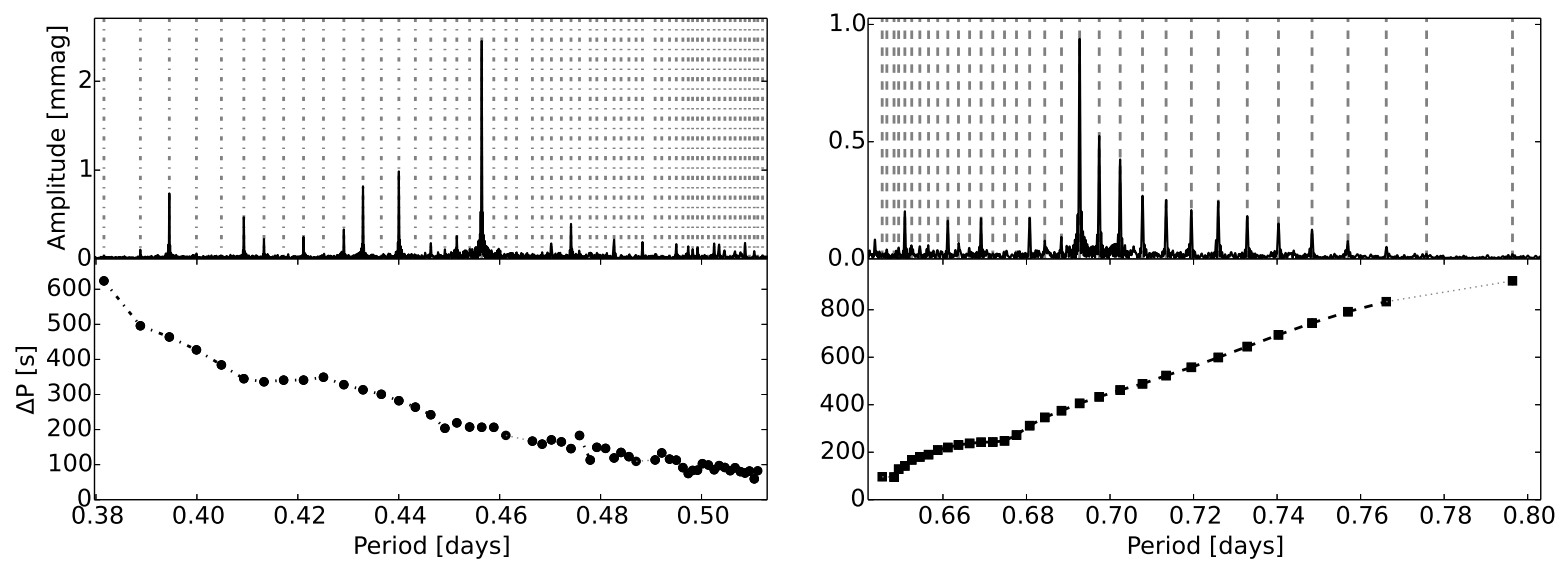

Fig. 41._ The period spacing patterns of KIC 9210943. 


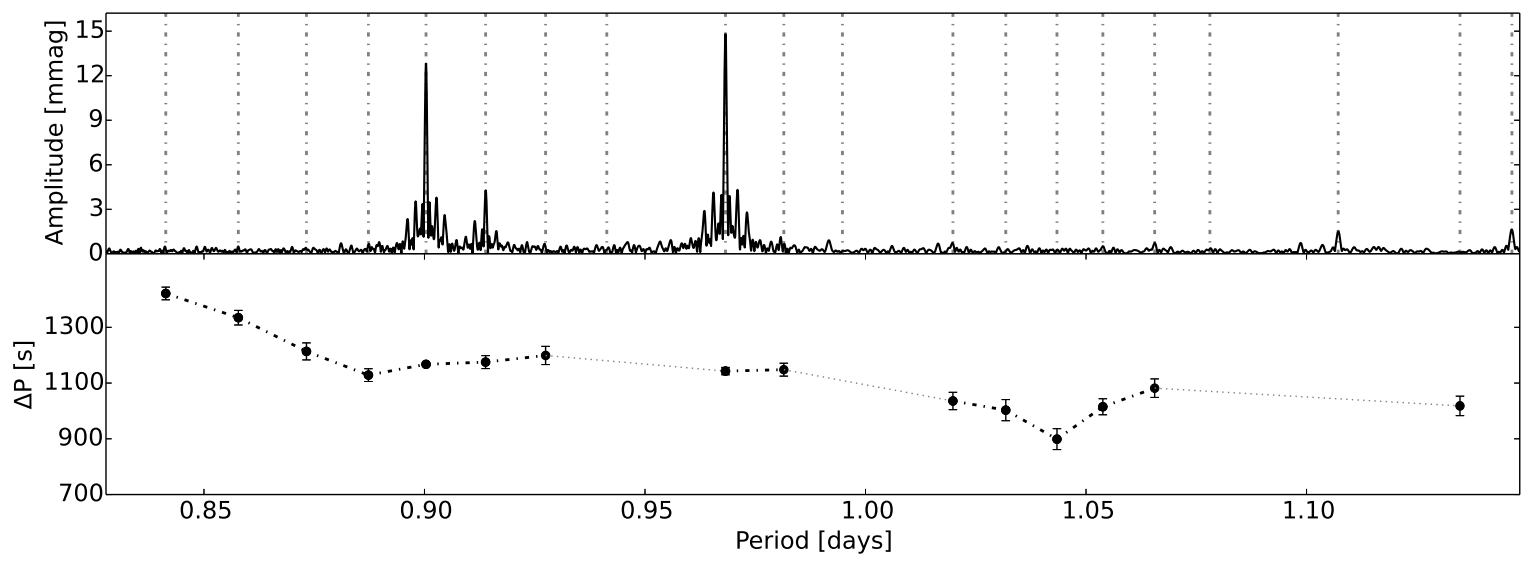

Fig. 42.- The period spacing patterns of KIC 9419694.
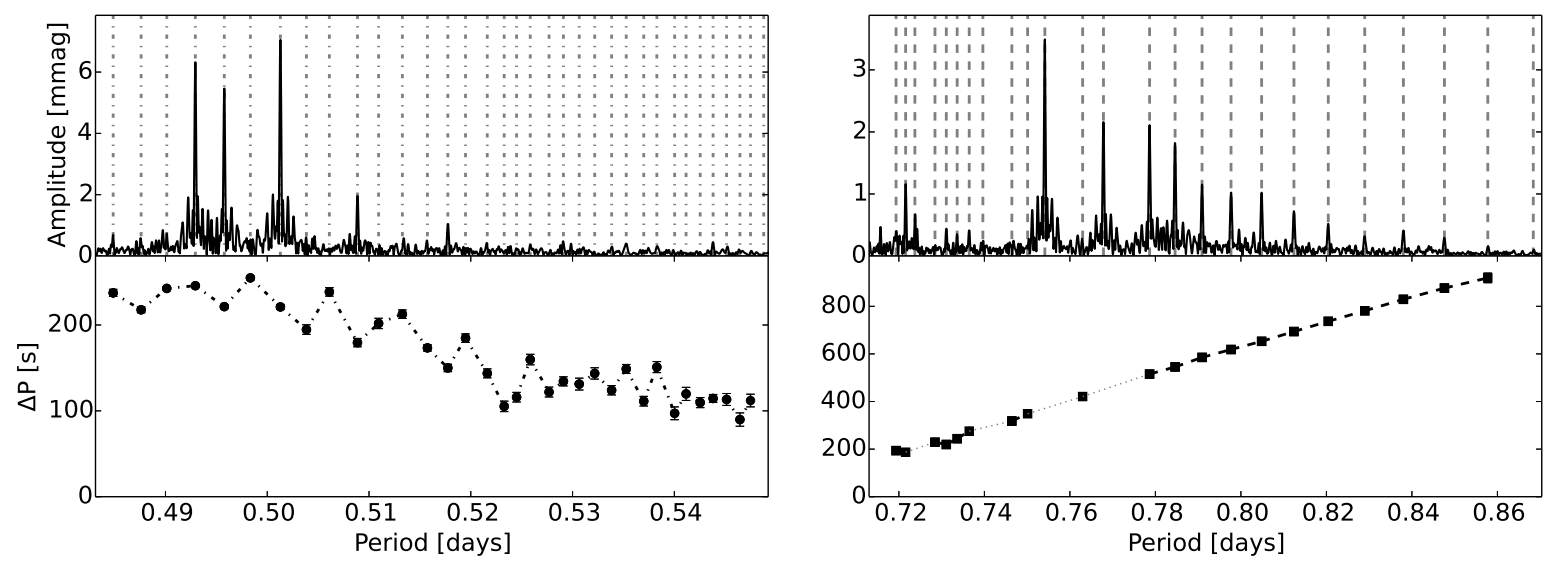

Fig. 43.- The period spacing patterns of KIC 9480469. 

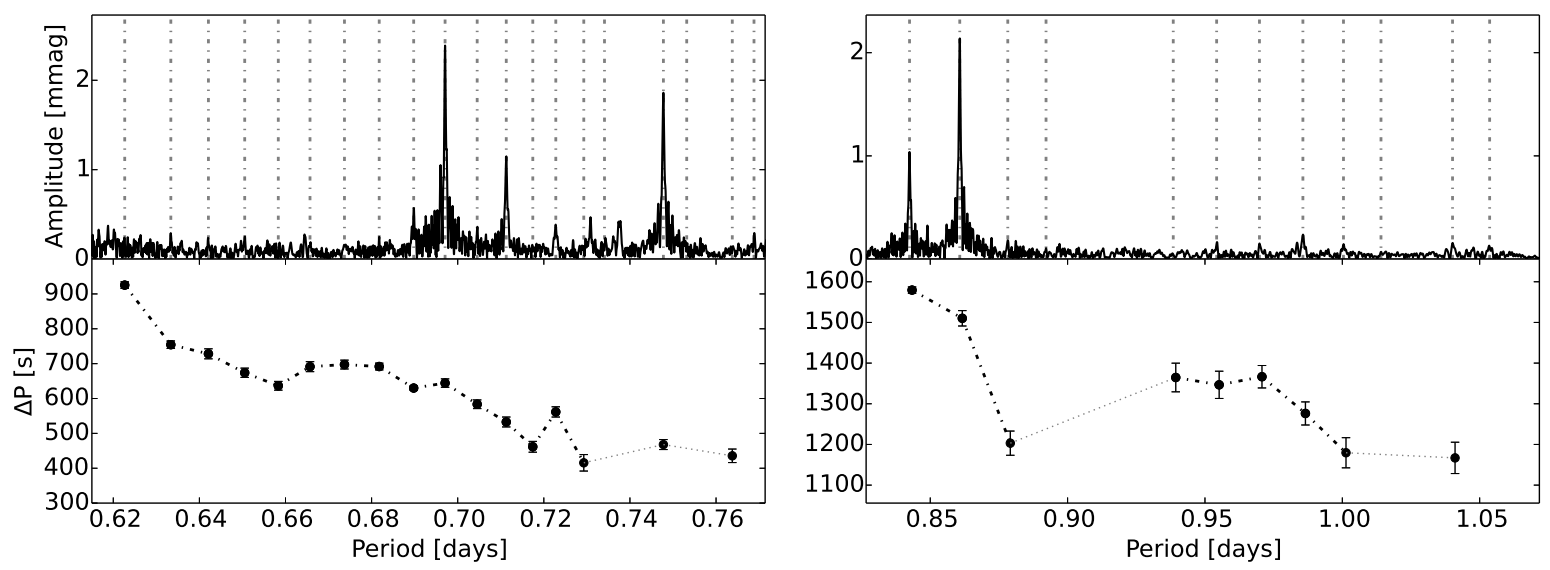

Fig. 44. - The period spacing patterns of KIC 9595743.

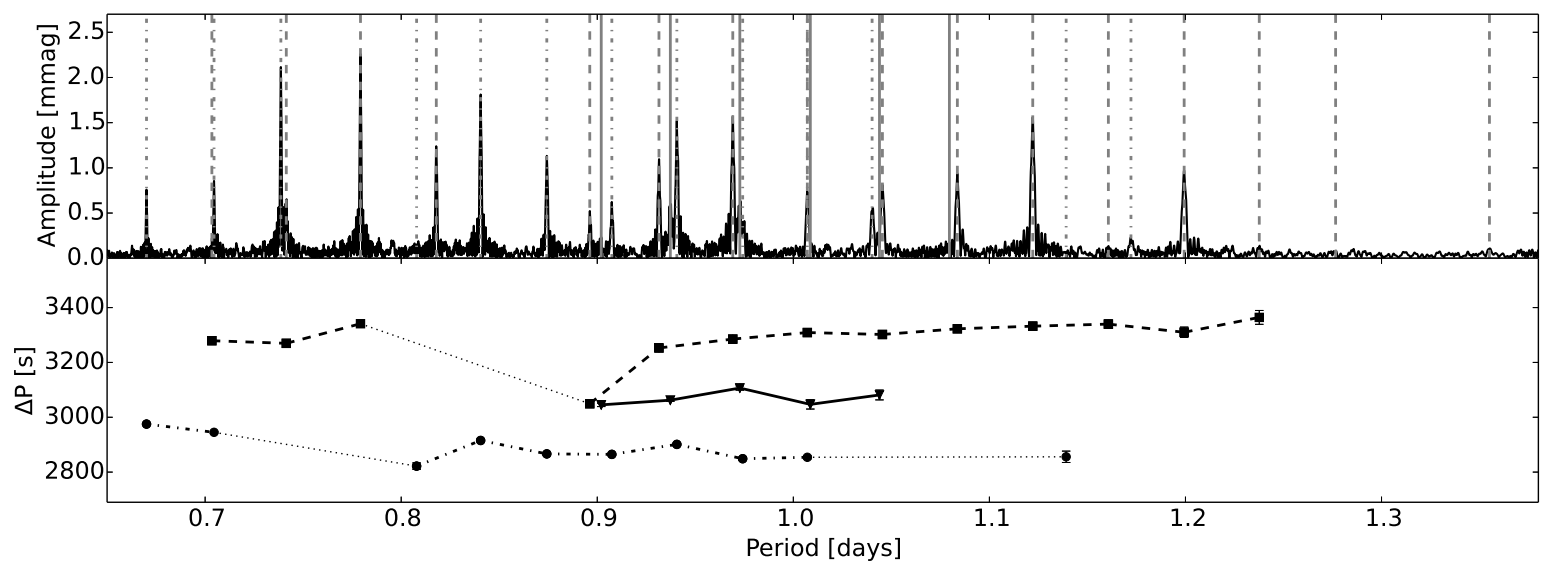

Fig. 45.- The period spacing patterns of KIC 9751996. 


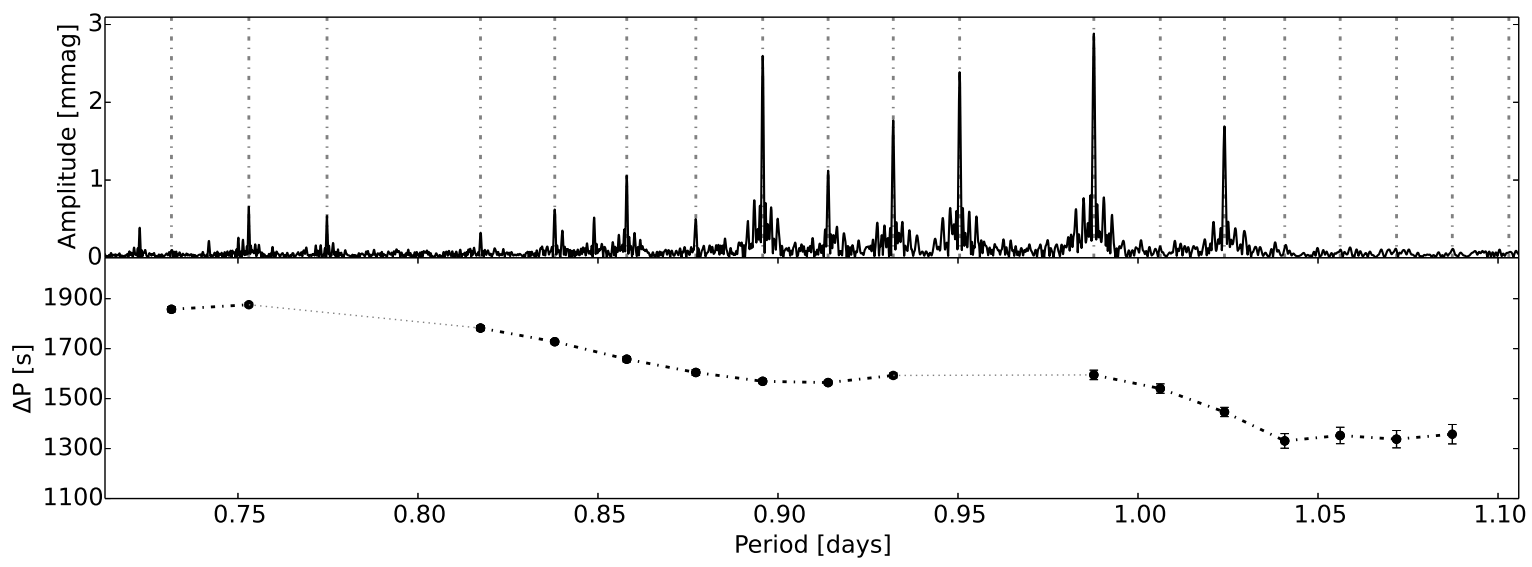

Fig. 46.- The period spacing patterns of KIC 10224094.

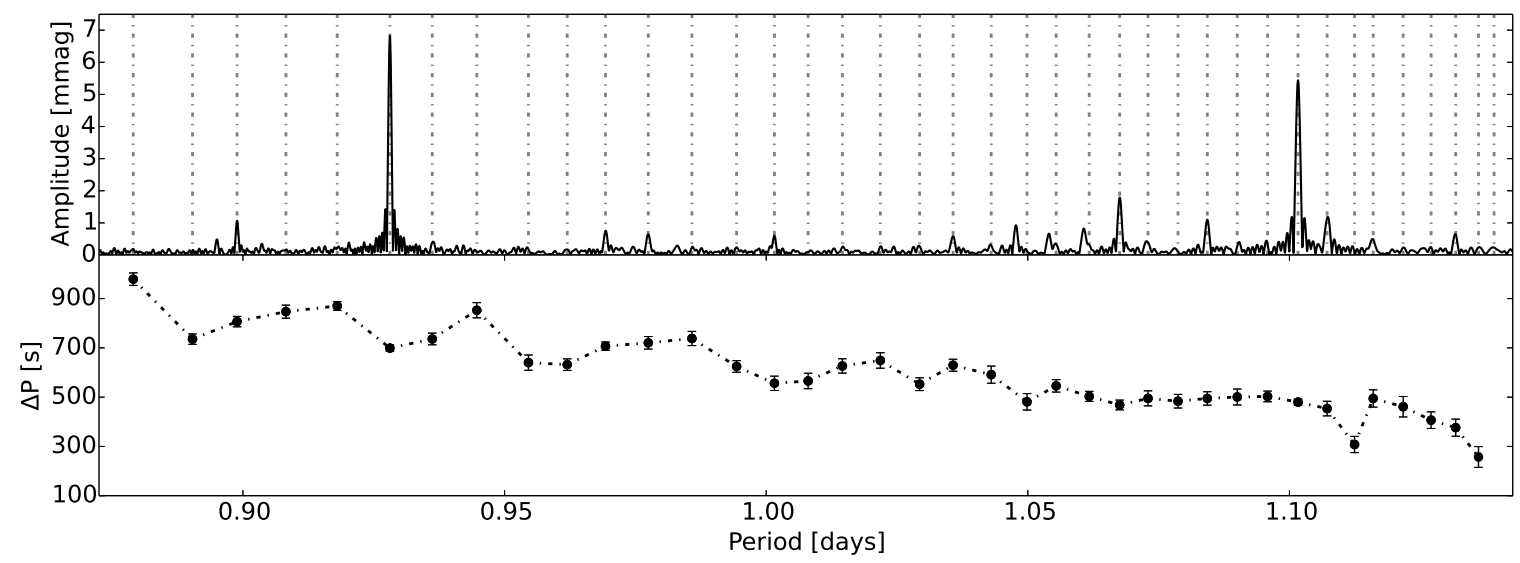

Fig. 47.- The period spacing patterns of KIC 10256787. 


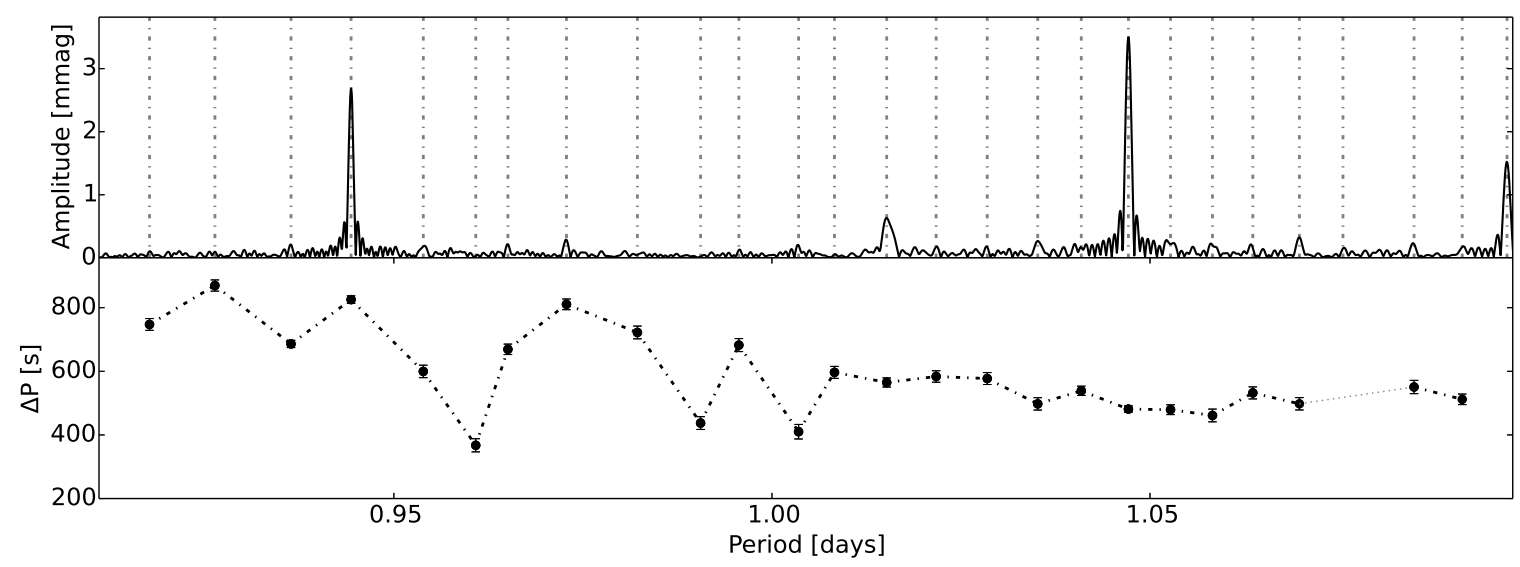

Fig. 48.- The period spacing patterns of KIC 10467146.

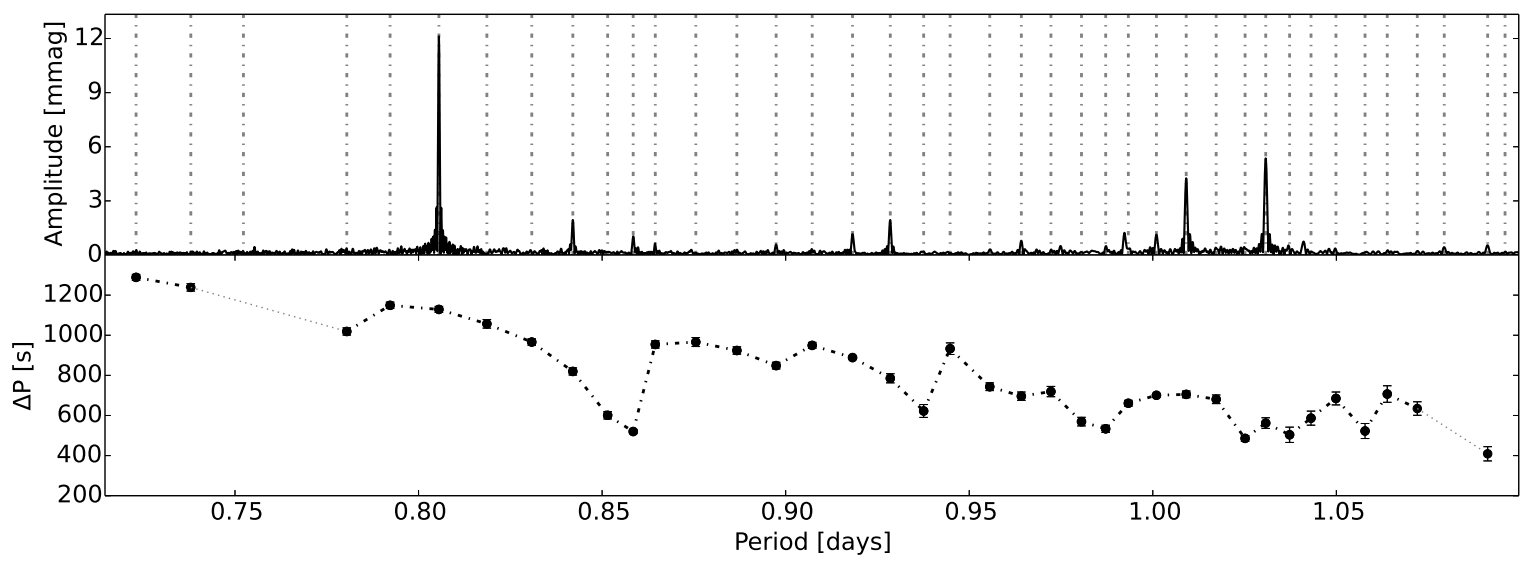

Fig. 49.- - The period spacing patterns of KIC 11080103. 


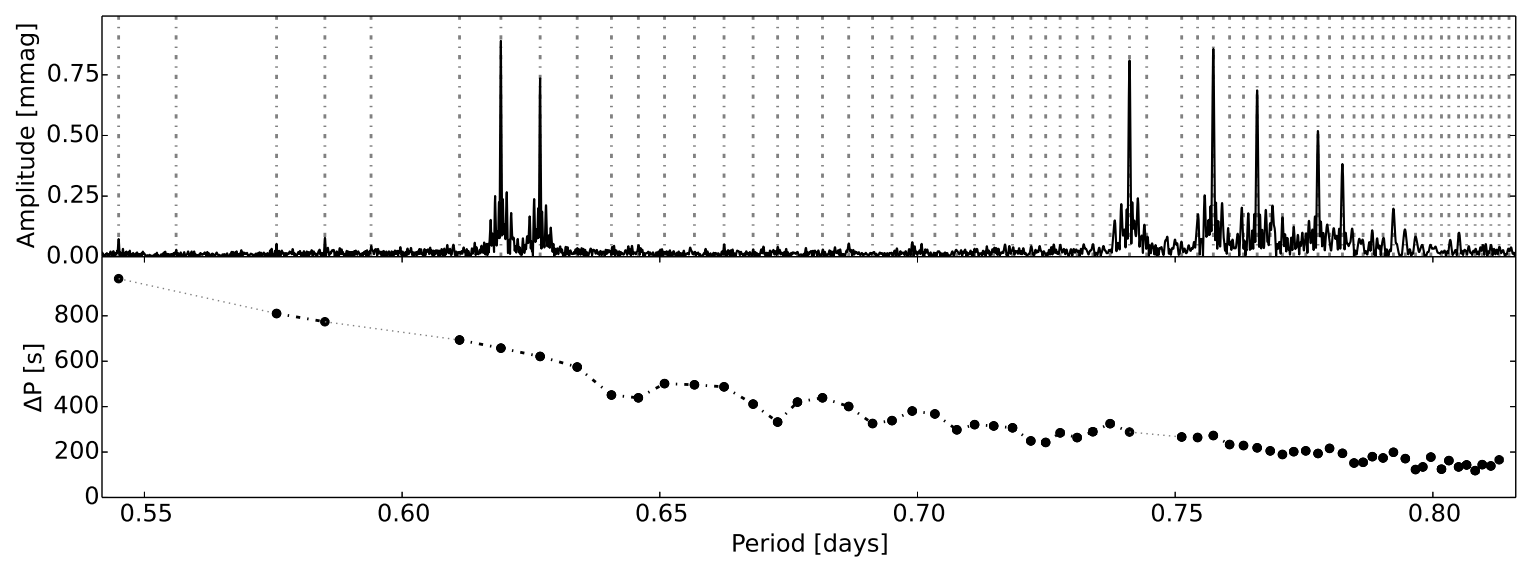

Fig. 50._ The period spacing patterns of KIC 11099031.
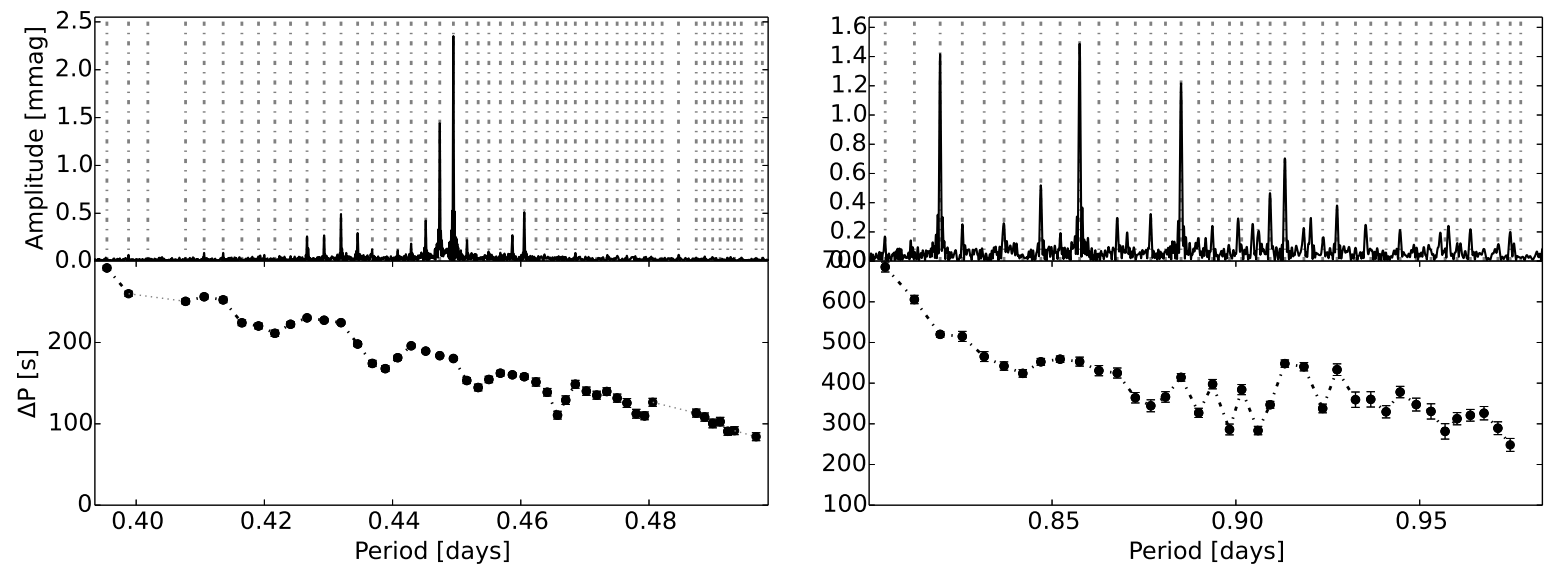

Fig. 51.— The period spacing patterns of KIC 11294808. 


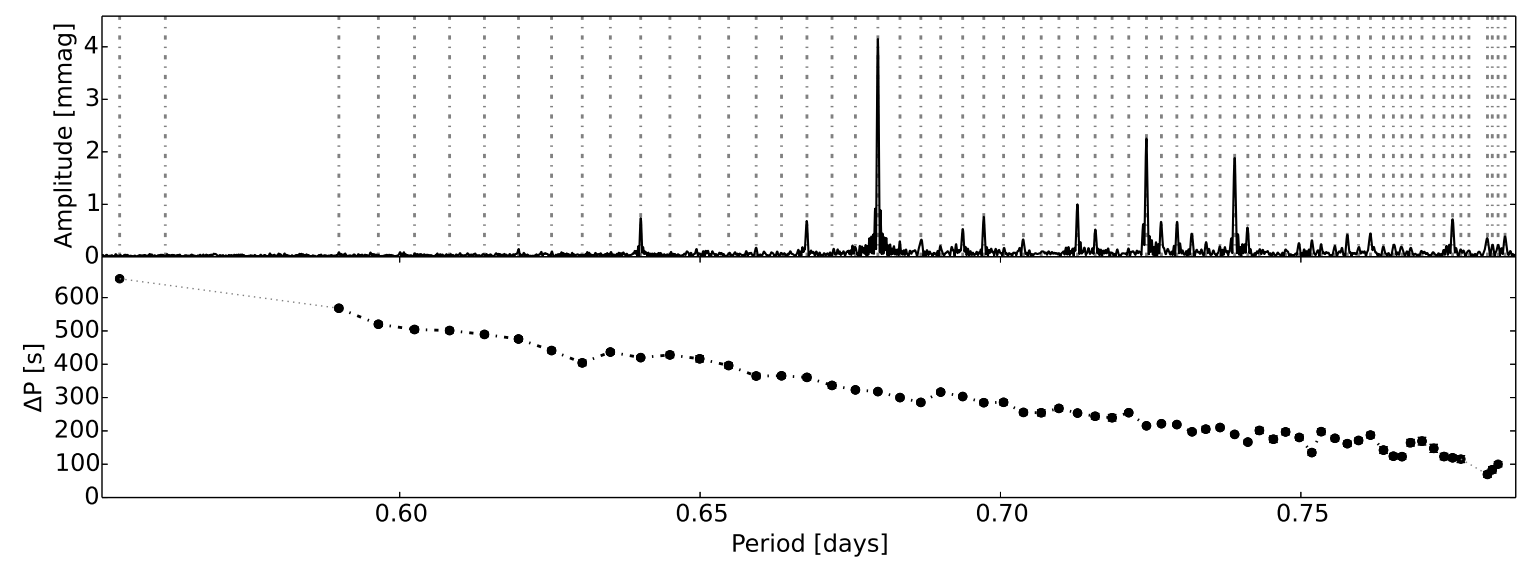

Fig. 52.- The period spacing patterns of KIC 11456474.

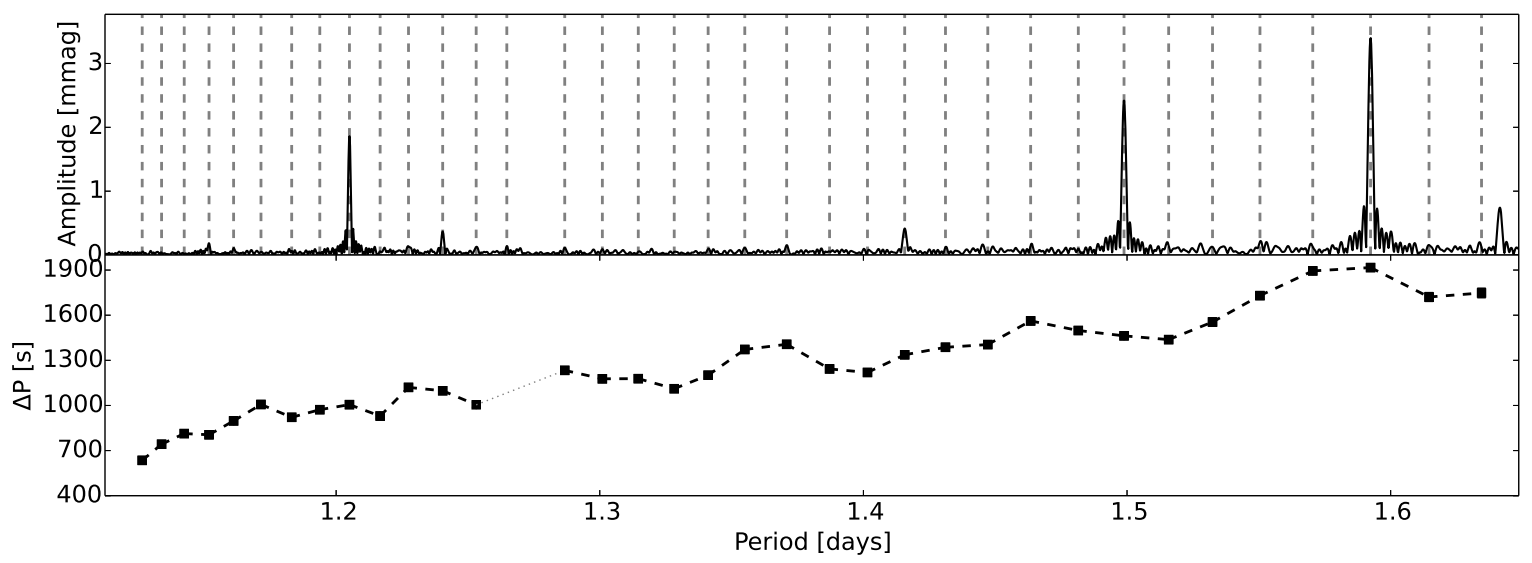

Fig. 53.- The period spacing patterns of KIC 11668783. 


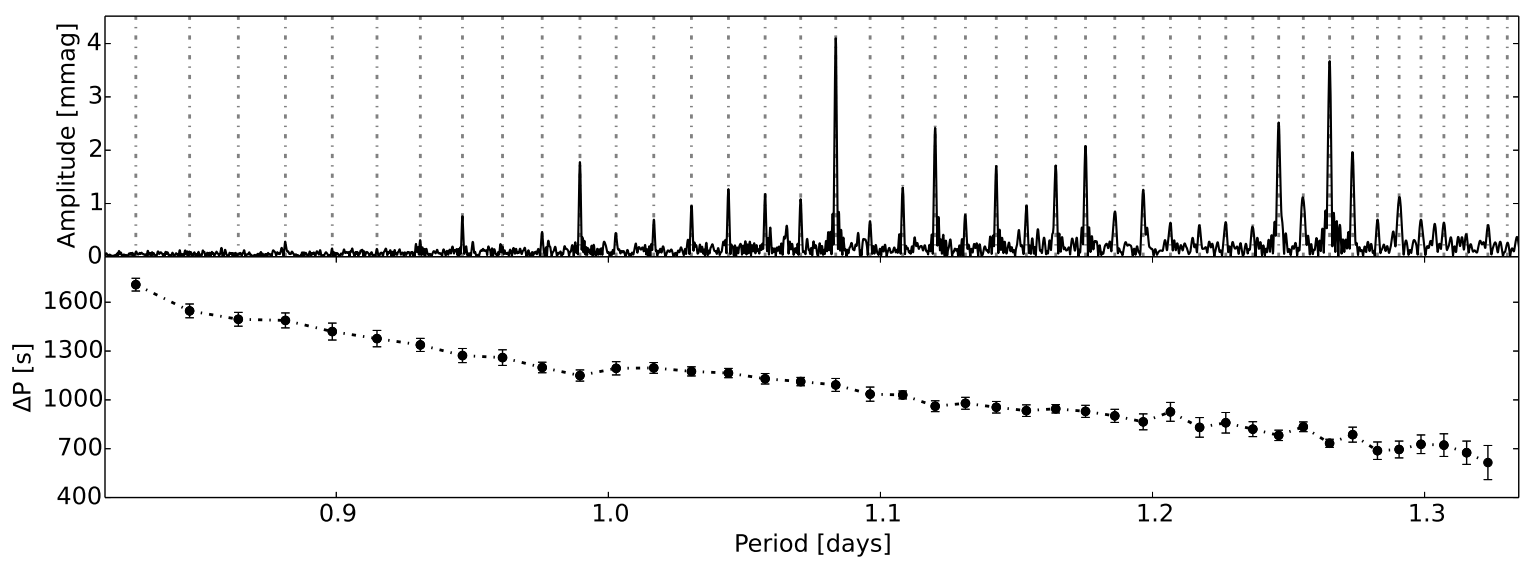

Fig. 54.- The period spacing patterns of KIC 11721304.

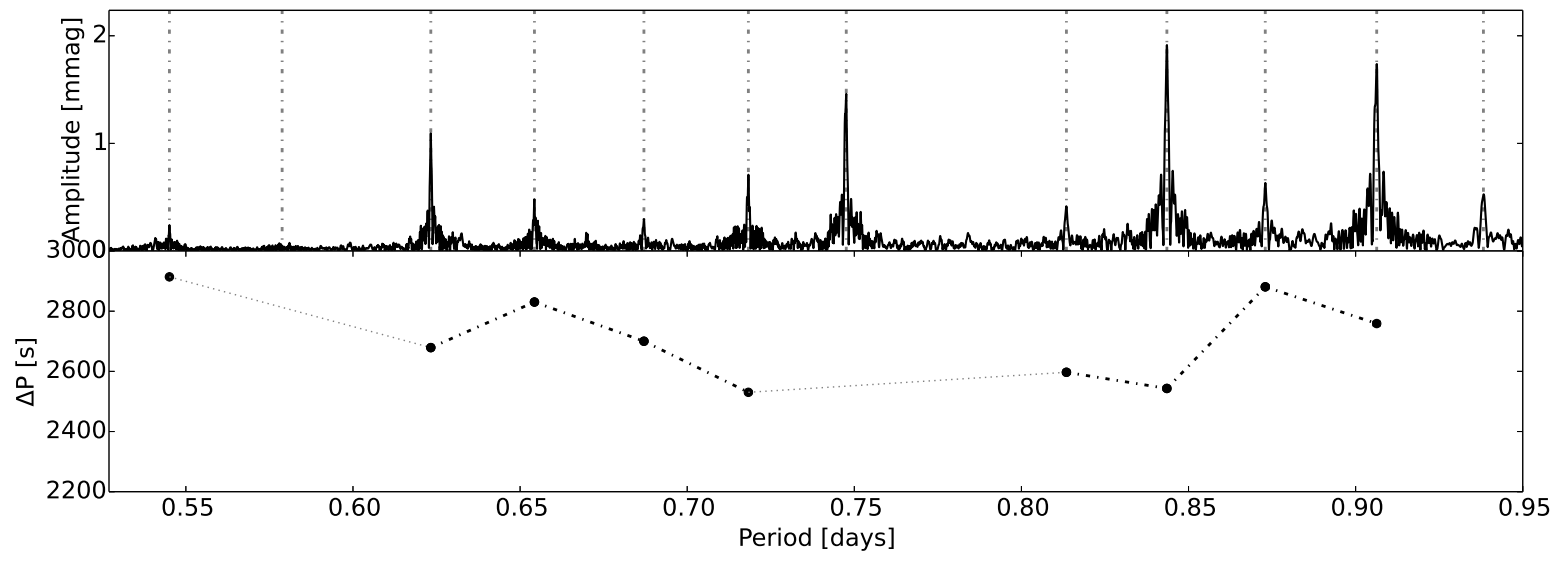

Fig. 55.- - The period spacing patterns of KIC 11754232. 


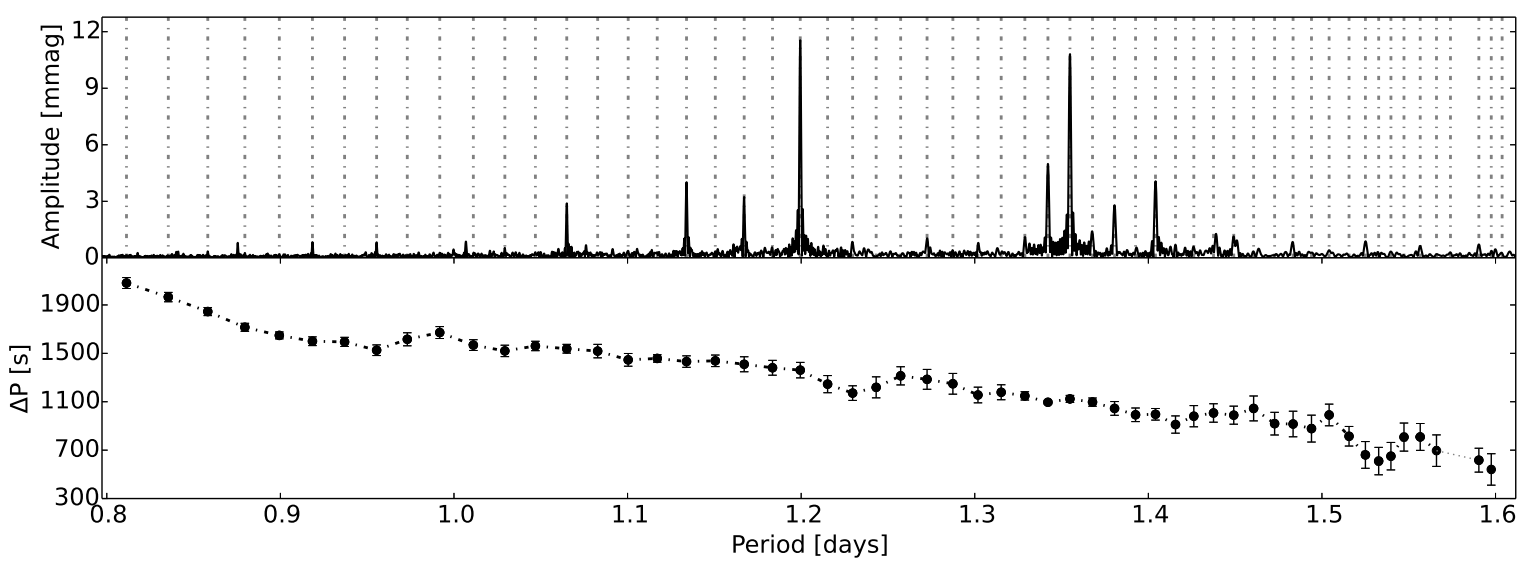

Fig. 56.- The period spacing patterns of KIC 11826272.
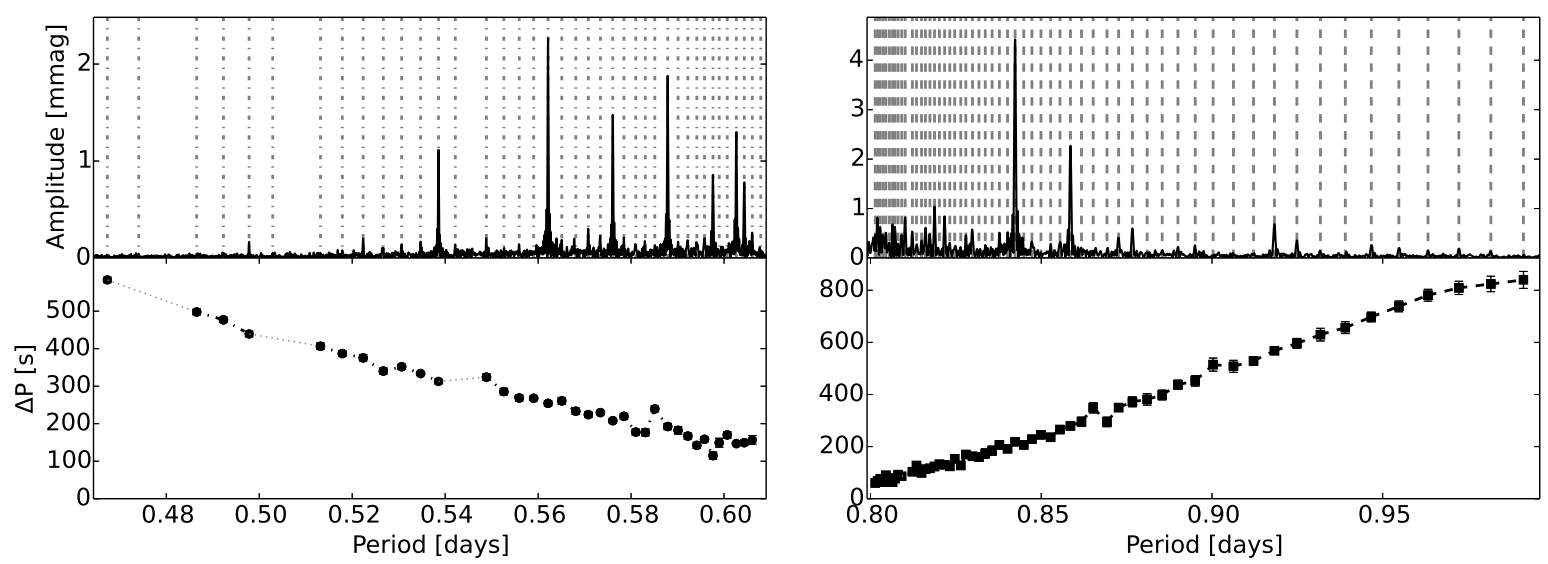

Fig. 57.— The period spacing patterns of KIC 11907454. 


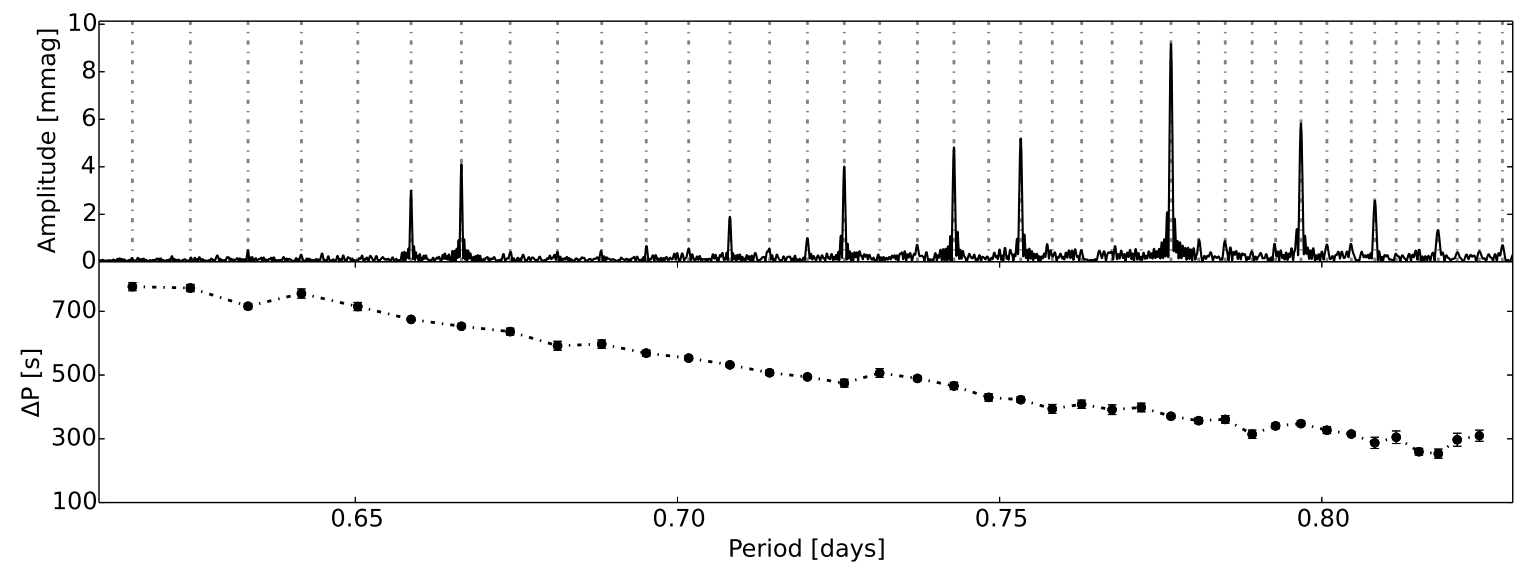

Fig. 58.- The period spacing patterns of KIC 11917550.

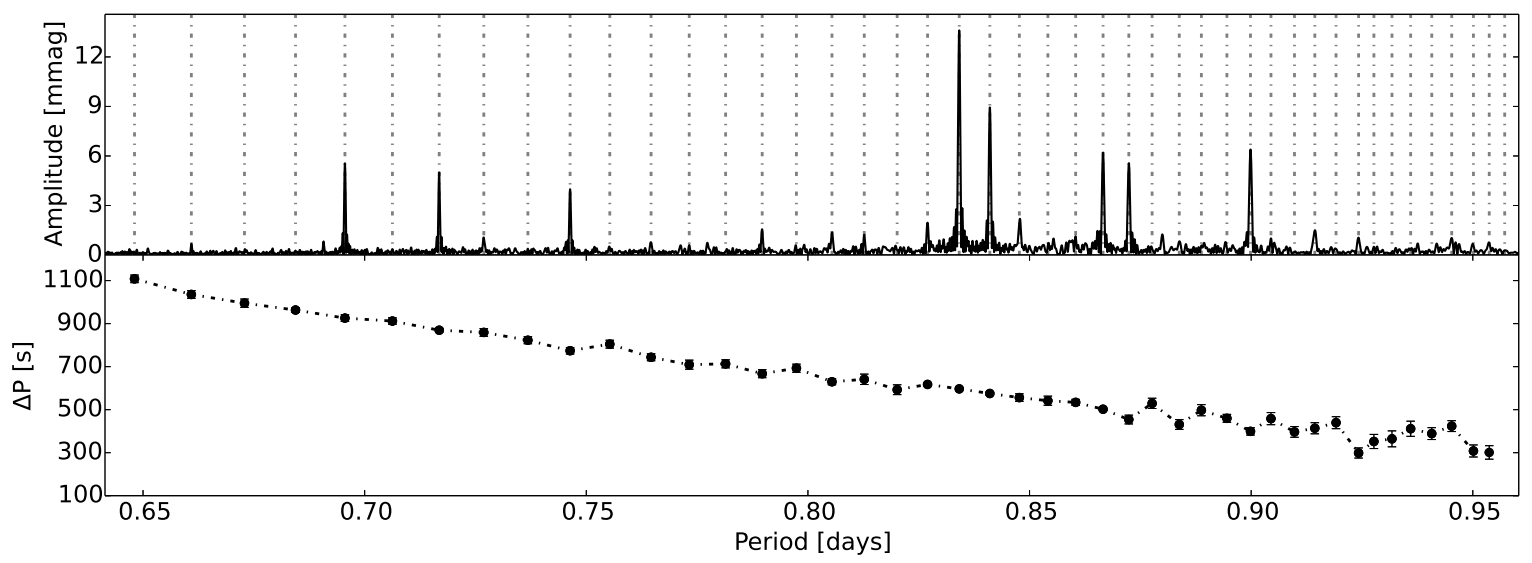

Fig. 59.- The period spacing patterns of KIC 11920505. 

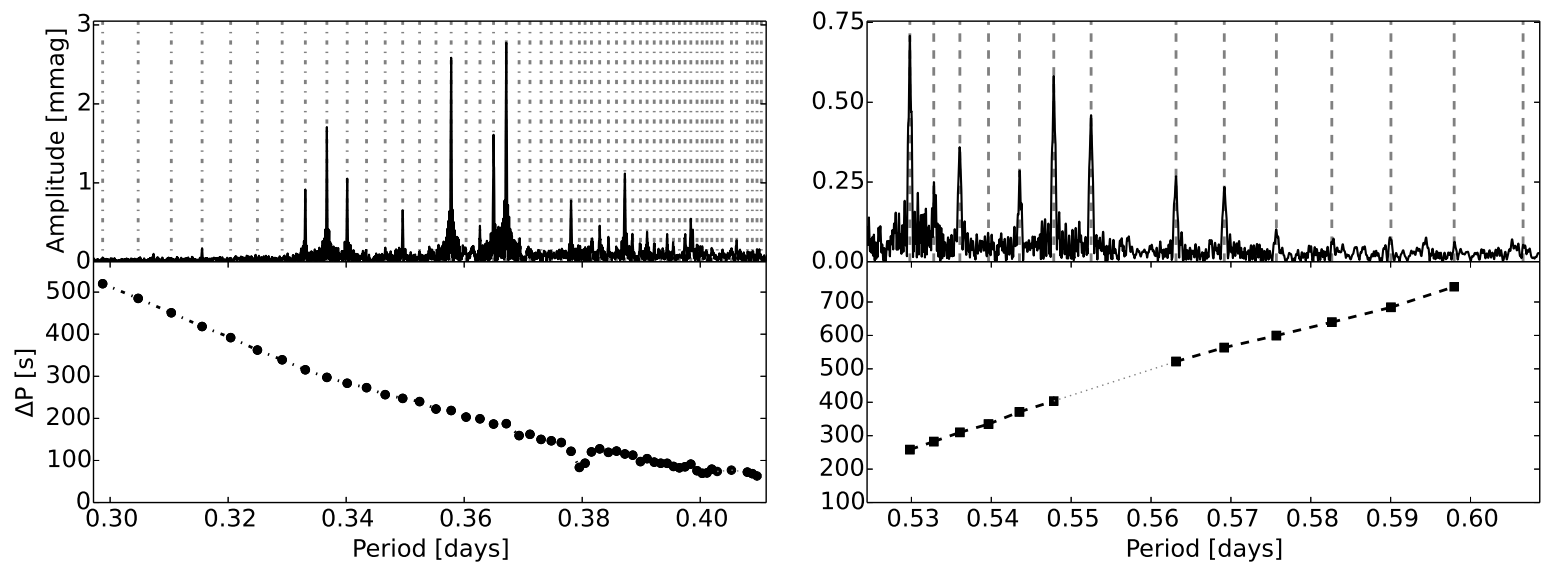

Fig. 60._ The period spacing patterns of KIC 12066947.

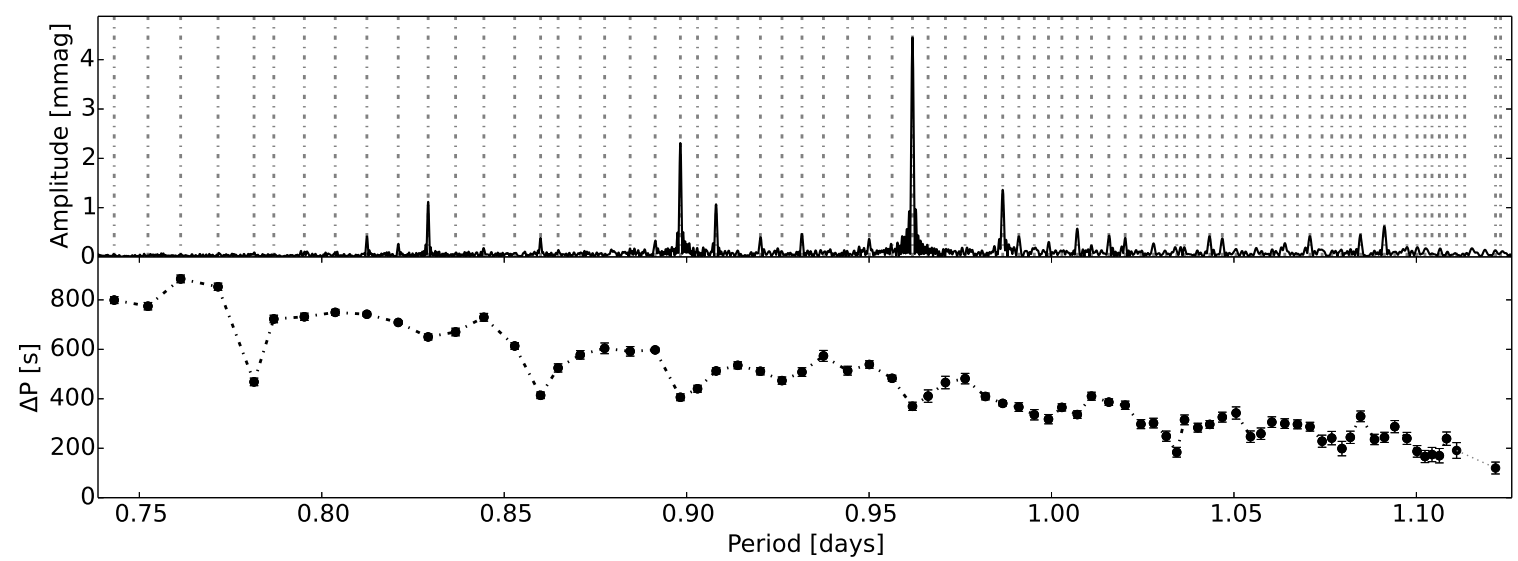

Fig. 61.- The period spacing patterns of KIC 12458189. 